\title{
Analysis of Discontinuous Dynamical Behaviors of a Friction-Induced Oscillator with an Elliptic Control Law
}

\author{
Jinjun Fan (D), Ping Liu, Tianyi Liu, Shan Xue, and Zhaoxia Yang \\ School of Mathematics and Statistics, Shandong Normal University, Jinan 250014, China \\ Correspondence should be addressed to Jinjun Fan; fjj18@126.com
}

Received 3 December 2017; Revised 24 February 2018; Accepted 15 March 2018; Published 8 May 2018

Academic Editor: Stefano Lenci

Copyright (c) 2018 Jinjun Fan et al. This is an open access article distributed under the Creative Commons Attribution License, which permits unrestricted use, distribution, and reproduction in any medium, provided the original work is properly cited.

\begin{abstract}
This paper develops the passability conditions of flow to the discontinuous boundary and the sticking or sliding and grazing conditions to the separation boundary in the discontinuous dynamical system of a friction-induced oscillator with an elliptic control law and the friction force acting on the mass $M$ through the analysis of the corresponding vector fields and $G$-functions. The periodic motions of such a discontinuous system are predicted analytically through the mapping structure. Finally, the numerical simulations are given to illustrate the analytical results of motion for a better understanding of physics of motion in the mass-spring-damper oscillator.
\end{abstract}

\section{Introduction}

Discontinuous dynamical systems extensively exist in mechanics and engineering, such as turbine blades, dry friction, and impact processes. Luo and Rapp $[1,2]$ investigated different periodic motions in a periodic force discontinuous system. The switching boundary in the aforementioned papers was supposed to be an inclined line or a parabolic curve and the friction force acting on the oscillator was not considered. In this paper, the motions in the discontinuous dynamical system of a friction-induced oscillator with an elliptic control law will be investigated.

In mechanical engineering, the friction contact between two surfaces of two bodies is an important connection and friction phenomenon widely exists. In recent years, much research effort in science and engineering has focussed on nonsmooth dynamical systems. This problem can go back to the 30s of last century. In 1930, Hartog [3] investigated the nonstick periodic motion of the forced linear oscillator with Coulomb and viscous damping. In 1960, Levitan [4] proved the existence of periodic motions in a friction oscillator with the periodically driven base. Discontinuous systems are usually described by ordinary differential equations with discontinuous right-hand sides. Filippov presented the basic concepts for such discontinuous differential equations and mainly discussed the existence and uniqueness of solutions for discontinuous dynamical systems in [5] in 1964 and gave the systematical summarization for such discontinuous differential equations in [6] in 1988. Since then, many authors have used and tried to extend the Filippov's theory to investigate the flow passability and motion complexity in discontinuous dynamical systems. For instance, in 1995, Popp et al. [7] investigated dynamical behavior of a friction oscillator with simultaneous self-excitation and external excitation. In 1996, Oestreich et al. [8] studied the bifurcation and stability analysis for a nonsmooth friction oscillator. In 1997, Kunze et al. [9] employed the KAM theory to analyze the periodic motions for a forced oscillator with a jump of restoring force. In 2003, Awrejcewicz and Olejnik [10] studied stickslip dynamics of a two-degree-of-freedom system. In 2003 and 2012, Cid and Sanchez [11] and Jacquemard and Teixeira [12] used an approximation procedure and the method of lower and upper solution to obtain the existence conditions of typical periodic solutions for some nonautonomous secondorder differential equations with a jump discontinuity. In 2001 and 2006, Kupper and Moritz [13] and Zou et al. [14] reported the possibility of Hopf bifurcations in planar discontinuous dynamical systems from the aspects of stability change of an equilibrium point. In 2014, Pascal [15] discussed a system composed of two masses connected by linear springs: one of 
the masses is in contact with a rough surface and the other is also subjected to a harmonic external force. Several periodic orbits were obtained in closed form, and symmetry in space and time was proved for some of these periodic solutions. For more discussion about discontinuous system, refer to [16-32].

On the other hand, the determination of periodic orbits along the switching boundary is very important in the nonsmooth control theory. In 2003, Bernardo et al. [33] showed that sliding bifurcations play an important role in analyzing the dynamics of dry friction oscillator. In 2004, Galvanetto [34] presented several detailed examples to address the fact that the occurrence of sliding bifurcation does not always correspond to the change in the number of periodic orbits at the bifurcation point. In 2005 and 2008, Kowalczyk et al. $[35,36]$ were concerned with the extension to the case of codimension 2 degenerate sliding bifurcations, numerical continuation, and analytical investigations of sliding bifurcations in Filippov systems. In 2010, Guardia et al. [37] analytically studied the sliding bifurcations of periodic orbits in a dry friction oscillator.

Recently, new development in discontinuous dynamical systems, such as the motion switchability for stick and sliding and grazing motions, has been found. In 2005, Luo [38, 39] developed a theory for nonsmooth dynamical systems on connectable domains and the mapping dynamics of periodic motions for a piecewise linear system under a periodic excitation. In 2005, 2006 and 2008, Luo [40-42] introduced the concepts of imaginary, sink, and source flows in nonsmooth dynamical systems and developed a general theory for the local singularity of a flow to the discontinuous boundary and a theory for flow switchability in discontinuous dynamical systems. In 2006, Luo and Gegg [43] investigated periodic motions in an oscillator moving on a periodically vibrating belt with dry friction. In 2009, Gegg et al. [44] presented the analytical conditions for sliding and passable motions on the periodically time-varying boundary for a friction-induced oscillator through the relative force product. In 2011, Luo [45] systematically presented a new theory for flow barriers in discontinuous dynamical systems, which provides a theoretic base to further develop control theory and stability. As to applications of the aforementioned local singularity theory, Luo and Huang [46] used such theory to determine the flow switchability on the discontinuous boundary for the nonlinear, friction-induced, periodically forced oscillator in 2012. In 2015 and 2017, Zhang and Fu [25, 47, 48] studied the periodic motions, stick motions, grazing flows in an inclined impact oscillator, and the flow switchability of motions in a horizontal impact pair with dry friction. In 2016, Chen and Fan [49] presented the analytical conditions for the motion switchability in a double belt friction oscillator system with a periodic excitation. In 2018, Fan et al. [50] further investigated dynamics of such double belt friction oscillator system with a periodic excitation. In 2017 and 2018, Fan et al. [51, 52] studied a friction-induced oscillator with two degrees of freedom on a speed-varying traveling belt and discontinuous dynamical behaviors in a vibroimpact system with multiple constraints, respectively.

In study of dynamical system, the switching control law also plays an important role. Some researchers have obtained some results by using the theory of discontinuous dynamical systems developed by Luo. In 2009, Luo and Rapp [1] investigated the flow switchability and periodic motions in a periodically forced, discontinuous dynamical system with switching control law of an inclined line. In the next year, Luo and Rapp [2] studied the motions and switchability of an oscillator in a periodically forced, discontinuous system with switching control law of a parabolic boundary. In 2015, Zheng and $\mathrm{Fu}$ [53] proposed the switched Van der Pol equation with impulsive effect as switched system and analyzed its features from a discontinuous point of view. In 2017, Fan et al. [54] studied discontinuous dynamics of a friction-induced oscillator with switching control law of a straight line. For the examples of impulsive control and Boolean control as switching control law, see [55-68]. It is worth noting that the switching control law of an elliptic boundary has an important application in practical problem, such as satellite rendezvous and formation flight. The elliptical controller is designed as a reference orbit for formation flight. By selecting the appropriate parameters to design the controller, the orbit of the satellite can be controlled so that it can conduct sliding or periodic motion in the reference orbit. This can effectively improve flight efficiency and save fuel. Compared with the circular reference orbit, the flight mode of elliptical reference orbit has the advantages which can reach the earth layer boundary region of space physics research, travel through specific spaces for longer periods of time, collect more information, and realize multipoint synchronous measurement by three-dimensional configuration and so on. Some scholars do a lot of work. For example, Senqupta [69] investigated elliptic rendezvous in the chaser satellite frame in 2012. In the same year, Chang et al. [70] studied the transfer of satellites between elliptic Keplerian orbits using Lyapunov stability theory. In 2013, Yan et al. [71] investigated pseudospectral feedback control for three-axis magnetic attitude stabilization in elliptic orbits. In 2012, Do [72] presented a design of cooperative controllers that force a group of $N$ mobile agents with an elliptical shape and with limited sensing ranges to perform a desired formation.

In this paper, the analytical conditions for motion switchability on the discontinuous boundaries in a periodically forced, discontinuous system with an elliptic control law and the friction force acting on the mass $M$ will be developed through the $G$-functions of the vector fields to the discontinuous boundaries. The rest of the paper is organized in the following manner. In Section 2, the physical model of a periodically forced, linear, friction-induced oscillator is introduced by using mechanical models. In Section 3, different domains and boundaries are defined due to the elliptic switching control law and friction discontinuities. The $G^{-}$ functions are introduced to discuss the motion switchability, and the analytical conditions for the switching conditions of the passable motions, stick or sliding motions, and grazing motions are derived mathematically in Section 4. In Section 5, the switching sets and two-dimensional mappings are defined to describe the complex motions in the friction-induced oscillator with an elliptic control law and the friction acting on the mass. Further, the periodic motions, such as sliding periodic motions and stick periodic motions, are analytically 


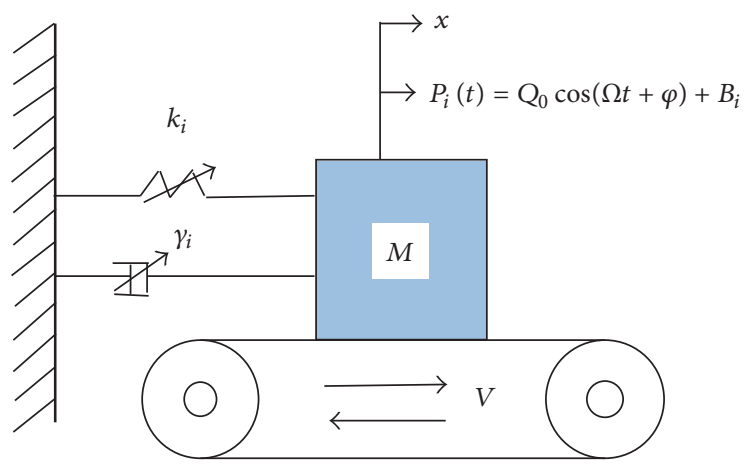

(a)

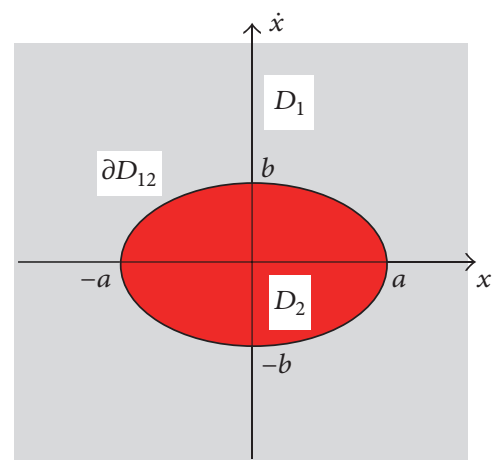

(b)

FIGURE 1: (a) Mechanical model; (b) elliptic control law's boundary.

predicted. Numerical simulations are carried out to illustrate the analytical conditions of motion switchability for a better understanding of the complex dynamical behaviors in Section 6. Finally, Section 7 concludes the paper.

\section{Mechanical Model}

Consider a periodically forced, linear, friction-induced oscillator, as shown in Figure 1(a). Denote displacement by $x$ and time by $t$, and assume that the switching boundary in this discontinuous dynamic system is

$$
\frac{x^{2}}{a^{2}}+\frac{\dot{x}^{2}}{b^{2}}=1
$$

where $a>0, b>0$, and $\dot{x}=d x / d t$, as sketched in Figure 1(b). This elliptical switching boundary will allow the system to switch from one dynamic system to another when the oscillator meets certain conditions. Assume also that the oscillator consists of a mass $M$, a switching spring of stiffness $k_{i}(i \in\{1,2\})$, and a switchable damper of coefficient $\gamma_{i}(i \in\{1,2\})$ in the domain $D_{i}(i \in\{1,2\})$, where $D_{1}$ is the unbounded outside domain and $D_{2}$ is the bounded inside domain. The periodic force acting on the mass $M$ in the different domains is

$$
P_{i}(t)=Q_{0} \cos (\Omega t+\varphi)+B_{i} \text { for } i \in\{1,2\},
$$

where $Q_{0}, \Omega, \varphi$, and $B_{i}$ are excitation amplitude, frequency, initial phase, and constant force, respectively. Through prestressing the mass $M$, the constant-force magnitude of $B_{i}$ could be adjusted to adapt to different working environments. Furthermore, the mass $M$ slides or rests on the conveyor belt with a constant speed $V$.

Since the mass $M$ contacts the conveyor belt with friction, the mass $M$ can move along or rest on the conveyor belt. Further, a kinetic friction force is given by

$$
F_{f}(\dot{x}) \begin{cases}=\mu F_{N}, & \dot{x}>V, \\ \in\left[-\mu F_{N}, \mu F_{N}\right], & \dot{x}=V, \\ =-\mu F_{N}, & \dot{x}<V,\end{cases}
$$

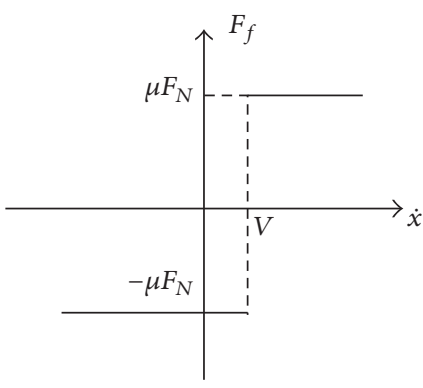

FIGURE 2: Friction force.

where $F_{N}=M g$ is the normal force between the mass $M$ and conveyor belt. The frictional force is sketched in Figure 2.

The equation of motion for such a friction-induced oscillator in the domain $D_{i}$ is

$$
\begin{array}{r}
\ddot{x}+2 d_{i} \dot{x}+c_{i} x=A_{0} \cos (\Omega t+\varphi)+b_{i}-f \operatorname{sgn}(\dot{x}-V) \\
\text { for } i \in\{1,2\},
\end{array}
$$

where

$$
\begin{aligned}
c_{i} & =\frac{k_{i}}{M}, \\
d_{i} & =\frac{\gamma_{i}}{2 M}, \\
A_{0} & =\frac{Q_{0}}{M}, \\
b_{i} & =\frac{B_{i}}{M}, \\
f & =\frac{\mu F_{N}}{M} .
\end{aligned}
$$

The nonfrictional force per unit mass in the domain $D_{i}$ is

$F_{\mathrm{nf}}^{(i)}=A_{0} \cos (\Omega t+\varphi)+b_{i}-2 d_{i} \dot{x}-c_{i} x$ 
The motions of the mass $M$ in the domain $D_{i}$ are divided into three cases.

(i) If $\left|F_{\mathrm{nf}}^{(i)}\right| \geq f_{f}^{(i)}$ and the displacement and velocity of the mass $M$ do not satisfy the elliptic control law (i.e., $x^{2} / a^{2}+$ $\dot{x}^{2} / b^{2} \neq 1$ ), the corresponding motion is called the nonstick motion or free-flight motion. For this case, the equation of motion is given as follows:

$$
\begin{array}{r}
\ddot{x}+2 d_{i} \dot{x}+c_{i} x=A_{0} \cos (\Omega t+\varphi)+b_{i}-f \operatorname{sgn}(\dot{x}-V) \\
\text { for } i \in\{1,2\},
\end{array}
$$

$$
\dot{x} \neq V \text {. }
$$

(ii) If the mass $M$ moves together with the conveyor belt (i.e., $\left|F_{\mathrm{nf}}^{(i)}\right|<f_{f}^{(i)}$ ) and the displacement and velocity of the mass $M$ do not satisfy the elliptic control law (i.e., $x^{2} / a^{2}+\dot{x}^{2} / b^{2} \neq 1$ ), the corresponding motion is called the stick motion. For this case, the equation of motion is given by

$$
\begin{aligned}
& \dot{x}=V, \\
& \ddot{x}=0 .
\end{aligned}
$$

(iii) If the displacement and velocity of the mass $M$ satisfy the elliptic control law (i.e., $x^{2} / a^{2}+\dot{x}^{2} / b^{2}=1$ ), the corresponding motion is called the sliding motion. For this case, the equation of motion is described by

$$
\frac{x^{2}}{a^{2}}+\frac{\dot{x}^{2}}{b^{2}}=1
$$

\section{Domains and Boundaries}

Due to the elliptic control law and the friction between the mass $M$ and the conveyor belt, the motions of the mass $M$ become discontinuous and more complicated. In order to determine the switching complexity for the motions of the mass $M$, different domains and boundaries in the absolute coordinates are defined in this section.

The origin of the absolute coordinates is set at the equilibrium position of the mass $M$. Based on the discontinuities caused by the switching control law and the friction between the mass $M$ and the conveyor belt, the phase plane for this discontinuous dynamic system is partitioned into four different domains and four boundaries, as shown in Figure 3. In each domain, the motion of the mass $M$ can be described through a continuous dynamical system.

Set

$$
\begin{aligned}
\varphi(x, \dot{x}) & =\frac{x^{2}}{a^{2}}+\frac{\dot{x}^{2}}{b^{2}}-1, \\
V & <b .
\end{aligned}
$$

The four nonstick domains for the mass $M$ can be expressed as

$$
\begin{aligned}
& \Omega_{1}=\left\{(x, \dot{x}) \mid \varphi(x, \dot{x})=\frac{x^{2}}{a^{2}}+\frac{\dot{x}^{2}}{b^{2}}-1>0, \dot{x}>V\right\}, \\
& \Omega_{2}=\left\{(x, \dot{x}) \mid \varphi(x, \dot{x})=\frac{x^{2}}{a^{2}}+\frac{\dot{x}^{2}}{b^{2}}-1<0, \dot{x}>V\right\},
\end{aligned}
$$

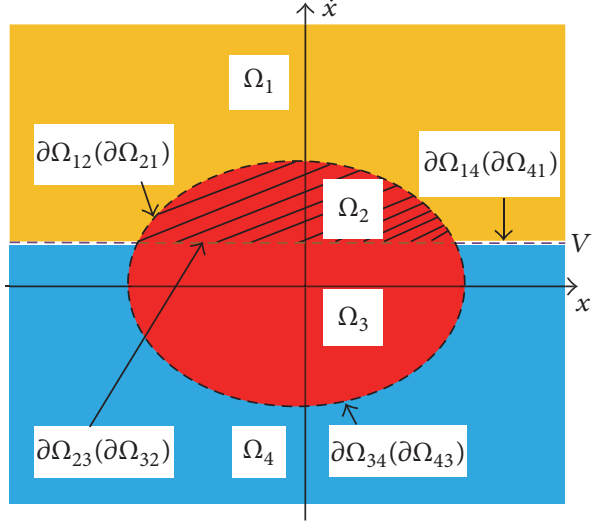

FIGURE 3: Domains and boundaries.

$$
\begin{aligned}
& \Omega_{3}=\left\{(x, \dot{x}) \mid \varphi(x, \dot{x})=\frac{x^{2}}{a^{2}}+\frac{\dot{x}^{2}}{b^{2}}-1<0, \dot{x}<V\right\}, \\
& \Omega_{4}=\left\{(x, \dot{x}) \mid \varphi(x, \dot{x})=\frac{x^{2}}{a^{2}}+\frac{\dot{x}^{2}}{b^{2}}-1>0, \dot{x}<V\right\} .
\end{aligned}
$$

The corresponding boundaries, including two stick or velocity boundaries and two sliding or control boundaries, can be defined as

$$
\begin{array}{r}
\partial \Omega_{12}=\partial \Omega_{21}=\bar{\Omega}_{1} \cap \bar{\Omega}_{2}=\left\{(x, \dot{x}) \mid \varphi_{12}(x, \dot{x})\right. \\
\left.=\varphi_{21}(x, \dot{x})=\frac{x^{2}}{a^{2}}+\frac{\dot{x}^{2}}{b^{2}}-1=0, \dot{x}-V>0\right\}, \\
\partial \Omega_{23}=\partial \Omega_{32}=\bar{\Omega}_{2} \cap \bar{\Omega}_{3}=\left\{(x, \dot{x}) \mid \varphi_{23}(x, \dot{x})\right. \\
\left.=\varphi_{32}(x, \dot{x})=\dot{x}-V=0, \frac{x^{2}}{a^{2}}+\frac{\dot{x}^{2}}{b^{2}}-1<0\right\}, \\
\partial \Omega_{34}=\partial \Omega_{43}=\bar{\Omega}_{3} \cap \bar{\Omega}_{4}=\left\{(x, \dot{x}) \mid \varphi_{34}(x, \dot{x})\right. \\
\left.=\varphi_{43}(x, \dot{x})=\frac{x^{2}}{a^{2}}+\frac{\dot{x}^{2}}{b^{2}}-1=0, \dot{x}-V<0\right\}, \\
\partial \Omega_{14}=\partial \Omega_{41}=\bar{\Omega}_{1} \cap \bar{\Omega}_{4}=\left\{(x, \dot{x}) \mid \varphi_{14}(x, \dot{x})\right. \\
\left.=\varphi_{41}(x, \dot{x})=\dot{x}-V=0, \frac{x^{2}}{a^{2}}+\frac{\dot{x}^{2}}{b^{2}}-1>0\right\} .
\end{array}
$$

For the nonstick motions in domains, two vectors are introduced as

$$
\begin{aligned}
& \mathbf{x}^{(\lambda)}=\left(x^{(\lambda)}, \dot{x}^{(\lambda)}\right)^{\mathrm{T}}=\left(x^{(\lambda)}, y^{(\lambda)}\right)^{\mathrm{T}}, \\
& \mathbf{F}^{(\lambda)}=\left(\dot{x}^{(\lambda)}, F^{(\lambda)}\right)^{\mathrm{T}}=\left(y^{(\lambda)}, F^{(\lambda)}\right)^{\mathrm{T}},
\end{aligned}
$$


where $\lambda=1,2,3,4$. Using (13), the equations of nonstick motions are rewritten in the vector form of

$$
\dot{\mathbf{x}}^{(\lambda)}=\mathbf{F}^{(\lambda)}\left(\mathbf{x}^{(\lambda)}, t\right) \quad \text { for } \lambda \in\{1,2,3,4\},
$$

where

$$
\begin{aligned}
& F^{(\lambda)}\left(\mathbf{x}^{(\lambda)}, t\right)=A_{0} \cos (\Omega t+\varphi)+b_{\lambda}-f \operatorname{sgn}(y-V) \\
& -2 d_{\lambda} y-c_{\lambda} x \\
& b_{1}=b_{4}=\frac{B_{1}}{M}, \\
& b_{2}=b_{3}=\frac{B_{2}}{M} \\
& d_{1}=d_{4}=\frac{\gamma_{1}}{2 M} \\
& d_{2}=d_{3}=\frac{\gamma_{2}}{2 M} \\
& c_{1}=c_{4}=\frac{k_{1}}{M} \text {, } \\
& c_{2}=c_{3}=\frac{k_{2}}{M} \text {. }
\end{aligned}
$$

For the stick motions on velocity boundaries, two vectors are introduced as

$$
\begin{aligned}
& \mathbf{x}^{\left(\alpha_{1} \alpha_{2}\right)}=\left(x^{\left(\alpha_{1} \alpha_{2}\right)}, \dot{x}^{\left(\alpha_{1} \alpha_{2}\right)}\right)^{\mathrm{T}}=\left(x^{\left(\alpha_{1} \alpha_{2}\right)}, y^{\left(\alpha_{1} \alpha_{2}\right)}\right)^{\mathrm{T}}, \\
& \mathbf{F}^{\left(\alpha_{1} \alpha_{2}\right)}=\left(\dot{x}^{\left(\alpha_{1} \alpha_{2}\right)}, F^{\left(\alpha_{1} \alpha_{2}\right)}\right)^{\mathrm{T}}=\left(y^{\left(\alpha_{1} \alpha_{2}\right)}, F^{\left(\alpha_{1} \alpha_{2}\right)}\right)^{\mathrm{T}},
\end{aligned}
$$

where $\left(\alpha_{1}, \alpha_{2}\right) \in\{(2,3),(3,2),(1,4),(4,1)\}$. The equations of stick motions are rewritten in the vector form of

$$
\begin{aligned}
\dot{\mathbf{x}}^{\left(\alpha_{1} \alpha_{2}\right)}= & \mathbf{F}^{\left(\alpha_{1} \alpha_{2}\right)}\left(\mathbf{x}^{\left(\alpha_{1} \alpha_{2}\right)}, t\right) \\
& \text { for }\left(\alpha_{1}, \alpha_{2}\right) \in\{(2,3),(3,2),(1,4),(4,1)\},
\end{aligned}
$$

where

$$
\begin{aligned}
& F^{\left(\alpha_{1} \alpha_{2}\right)}\left(\mathbf{x}^{\left(\alpha_{1} \alpha_{2}\right)}, t\right)=0 \text { for stick motion, } \\
& F^{\left(\alpha_{1} \alpha_{2}\right)}\left(\mathbf{x}^{\left(\alpha_{1} \alpha_{2}\right)}, t\right) \in\left[F^{\left(\alpha_{1}\right)}\left(\mathbf{x}^{\left(\alpha_{1}\right)}, t\right), F^{\left(\alpha_{2}\right)}\left(\mathbf{x}^{\left(\alpha_{2}\right)}, t\right)\right]
\end{aligned}
$$

for nonstick motion.

For the sliding motions on elliptic boundaries, two vectors are introduced as

$$
\begin{aligned}
& \mathbf{x}^{\left(\alpha_{3} \alpha_{4}\right)}=\left(x^{\left(\alpha_{3} \alpha_{4}\right)}, \dot{x}^{\left(\alpha_{3} \alpha_{4}\right)}\right)^{\mathrm{T}}=\left(x^{\left(\alpha_{3} \alpha_{4}\right)}, y^{\left(\alpha_{3} \alpha_{4}\right)}\right)^{\mathrm{T}}, \\
& \mathbf{F}^{\left(\alpha_{3} \alpha_{4}\right)}=\left(\dot{x}^{\left(\alpha_{3} \alpha_{4}\right)}, F^{\left(\alpha_{3} \alpha_{4}\right)}\right)^{\mathrm{T}}=\left(y^{\left(\alpha_{3} \alpha_{4}\right)}, F^{\left(\alpha_{3} \alpha_{4}\right)}\right)^{\mathrm{T}},
\end{aligned}
$$

where $\left(\alpha_{3}, \alpha_{4}\right) \in\{(1,2),(2,1),(4,3),(3,4)\}$. The equations of sliding motions are rewritten in the vector form of

$$
\begin{aligned}
\dot{\mathbf{x}}^{\left(\alpha_{3} \alpha_{4}\right)}= & \mathbf{F}^{\left(\alpha_{3} \alpha_{4}\right)}\left(\mathbf{x}^{\left(\alpha_{3} \alpha_{4}\right)}, t\right) \\
& \text { for }\left(\alpha_{3}, \alpha_{4}\right) \in\{(1,2),(2,1),(4,3),(3,4)\},
\end{aligned}
$$

where

$$
\begin{aligned}
& F^{\left(\alpha_{3} \alpha_{4}\right)}\left(\mathbf{x}^{\left(\alpha_{3} \alpha_{4}\right)}, t\right)=-\frac{b^{2}}{a^{2}} x^{\left(\alpha_{3} \alpha_{4}\right)} \quad \text { for sliding motion, } \\
& F^{\left(\alpha_{3} \alpha_{4}\right)}\left(\mathbf{x}^{\left(\alpha_{3} \alpha_{4}\right)}, t\right) \in\left[F^{\left(\alpha_{3}\right)}\left(\mathbf{x}^{\left(\alpha_{3}\right)}, t\right), F^{\left(\alpha_{4}\right)}\left(\mathbf{x}^{\left(\alpha_{4}\right)}, t\right)\right]
\end{aligned}
$$

for non-sliding motion.

\section{Analytical Conditions}

According to the theory of flow switchability to a specific boundary in discontinuous dynamic systems, the switching conditions of the passable motions, stick or sliding motions, and grazing motions of the mass $M$ will be developed in this section.

4.1. Basic Theory. For convenience, the concepts of the Gfunctions and some lemmas are given in the following (see [42]).

Consider a dynamic system consisting of $N$ subdynamic systems in a universal domain $\mho \in \mathfrak{R}^{n}$. The universal domain is divided into $N$ accessible subdomains $\Omega_{i}$ and inaccessible subdomains $\Omega_{0}$. And the union of all the accessible subdomains is $\bigcup_{i=1}^{N} \Omega_{i}$ and the universal domain is $\mho=\bigcup_{i=1}^{N} \Omega_{i} \cup$ $\Omega_{0}$. On the $i$ th open subdomain $\Omega_{i}$, there is a $C^{r_{i}}$-continuous system $\left(r_{i} \geq 1\right)$ in the form of

$$
\begin{aligned}
\dot{\mathbf{x}}^{(i)} & \equiv \mathbf{F}^{(i)}\left(\mathbf{x}^{(i)}, t, \mathbf{P}_{i}\right) \in R^{n}, \\
\mathbf{x}^{(i)} & =\left(x_{1}^{(i)}, x_{2}^{(i)}, \ldots, x_{n}^{(i)}\right)^{\mathrm{T}} \in \Omega_{i},
\end{aligned}
$$

where the time is $t$ and $\dot{\mathbf{x}}^{(i)}=d \mathbf{x}^{(i)} / d t$. In an accessible subdomain $\Omega_{i}$, the vector field $\mathbf{F}^{(i)}\left(\mathbf{x}^{(i)}, t, \mathbf{P}_{i}\right)$ with a parameter vector $\mathbf{P}_{i}=\left(p_{i}^{(1)}, p_{i}^{(2)}, \ldots, p_{i}^{(l)}\right)^{\mathrm{T}} \in R^{l}$ is $C^{r_{i}}$-continuous $\left(r_{i} \geq\right.$ 1) for all time $t$, and the flow in (22) $\mathbf{x}_{t}^{(i)}=\Phi^{(i)}\left(t_{0}, \mathbf{x}_{0}^{(i)}, \mathbf{P}_{i}, t\right)$ with an initial condition $\left(t_{0}, \mathbf{x}_{0}^{(i)}\right)$ is $C^{r_{i}+1}$ continuous for time $t$, and on the boundary of two adjacent domains $\partial \Omega_{\alpha \beta}=$ $\bar{\Omega}_{\alpha} \cap \bar{\Omega}_{\beta}=\left\{\mathbf{x} \mid \varphi_{\alpha \beta}(\mathbf{x}, t, \lambda)=0, \varphi_{\alpha \beta}\right.$ is $C^{r}$-continuous $(r \geq 1)\} \subset R^{n-1}$, and there is a flow $\mathbf{x}_{t}^{(0)}=\Phi^{(0)}\left(t_{0}, \mathbf{x}_{0}^{(0)}, \lambda, t\right)$, which can be determined by

$$
\begin{aligned}
& \dot{\mathbf{x}}^{(0)} \equiv \mathbf{F}^{(0)}\left(\mathbf{x}^{(0)}, t, \lambda\right) \in R^{n}, \\
& \mathbf{x}^{(0)}=\left(x_{1}^{(0)}, x_{2}^{(0)}, \ldots, x_{n}^{(0)}\right)^{\mathrm{T}} \in \partial \Omega_{\alpha \beta}
\end{aligned}
$$

with an initial condition $\left(t_{0}, \mathbf{x}_{0}^{(0)}\right)$ and a parameter vector $\lambda=$ $\left(\lambda_{1}, \lambda_{2}, \ldots, \lambda_{k}\right)^{\mathrm{T}} \in R^{k}$.

Assume that there are two adjacent subdomains $\Omega_{\alpha}$ and $\Omega_{\beta}$. A flow in subdomain $\Omega_{i}(i \in\{\alpha, \beta\})$ is called a local flow if it is governed by the continuous vector field in $\Omega_{i}(i \in$ $\{\alpha, \beta\})$ only. A flow is called a passable flow if it switches from a subdomain $\Omega_{i}(i \in\{\alpha, \beta\})$ into another one $\Omega_{j}(j \in$ $\{\alpha, \beta\}, j \neq i)$ through the boundary $\partial \Omega_{i j}(i, j \in\{\alpha, \beta\}, i \neq$ $j)$. Obviously, the vector field of a passable flow in subdomain $\Omega_{i}(i \in\{\alpha, \beta\})$ will be changed into the one in subdomain $\Omega_{j}(j \in\{\alpha, \beta\}, j \neq i)$, accordingly. A flow is called a grazing 
flow if it is tangential to the boundaries $\partial \Omega_{\alpha \beta}$. If a flow cannot pass through but moves along the boundaries $\partial \Omega_{\alpha \beta}$, then this flow is called a sliding flow.

Definition 1 (see [42]). The 0th-order $G$-functions of the domain flow $\mathbf{x}_{t}^{(i)}(i \in\{\alpha, \beta\})$ to the boundary flow $\mathbf{x}_{t}^{(0)}$ on the boundary in the normal direction of the boundary $\partial \Omega_{\alpha \beta}$ are defined as

$$
\begin{gathered}
G_{\partial \Omega_{\alpha \beta}}^{(i)}\left(\mathbf{x}_{t}^{(0)}, t_{ \pm}, \mathbf{x}_{t_{ \pm}}^{(i)}, \mathbf{P}_{i}, \lambda\right) \equiv G_{\partial \Omega_{\alpha \beta}}^{(0, i)}\left(\mathbf{x}_{t}^{(0)}, t_{ \pm}, \mathbf{x}_{t_{ \pm}}^{(i)}, \mathbf{P}_{i}, \lambda\right) \\
=D_{\mathbf{x}_{t}(0)}{ }^{t}{ }_{n_{\partial \Omega_{\alpha \beta}}}^{\mathrm{T}} \cdot\left(\mathbf{x}_{t_{ \pm}}^{(i)}-\mathbf{x}_{t}^{(0)}\right)+{ }^{t} \mathbf{n}_{\partial \Omega_{\alpha \beta}}^{\mathrm{T}} \cdot\left(\dot{\mathbf{x}}_{t_{ \pm}}^{(i)}-\dot{\mathbf{x}}_{t}^{(0)}\right) .
\end{gathered}
$$

Definition 2 (see [42]). The 1st-order G-functions of the domain flow $\mathbf{x}_{t}^{(i)}(i \in\{\alpha, \beta\})$ to the boundary flow $\mathbf{x}_{t}^{(0)}$ on the boundary in the normal direction of the boundary $\partial \Omega_{\alpha \beta}$ are defined as

$$
\begin{aligned}
G_{\partial \Omega_{\alpha \beta}}^{(1, i)} & \left(\mathbf{x}_{t}^{(0)}, t_{ \pm}, \mathbf{x}_{t_{ \pm}}^{(i)}, \mathbf{P}_{i}, \lambda\right) \\
= & D_{\mathbf{x}_{t}^{(0)}}^{2}{ }^{t} \mathbf{n}_{\partial \Omega_{\alpha \beta}}^{\mathrm{T}} \cdot\left(\mathbf{x}_{t_{ \pm}}^{(i)}-\mathbf{x}_{t}^{(0)}\right)+2 D_{\mathbf{x}_{t}^{(0)}}{ }^{t} \mathbf{n}_{\partial \Omega_{\alpha \beta}}^{\mathrm{T}} \\
& \cdot\left(\dot{\mathbf{x}}_{t_{ \pm}}^{(i)}-\dot{\mathbf{x}}_{t}^{(0)}\right)+{ }^{t} \mathbf{n}_{\partial \Omega_{\alpha \beta}}^{\mathrm{T}} \cdot\left(\ddot{\mathbf{x}}_{t_{ \pm}}^{(i)}-\ddot{\mathbf{x}}_{t}{ }^{(0)}\right) .
\end{aligned}
$$

In above definitions, the total derivative $D_{\mathbf{x}_{t}^{(0)}}(\cdot)$ := $\partial(\cdot) / \partial \mathbf{x}_{t}^{(0)} \cdot \dot{\mathbf{x}}_{t}^{(0)}+\partial(\cdot) / \partial t$, and the normal vector of the boundary surface $\partial \Omega_{\alpha \beta}$ at point $\mathbf{x}_{t}^{(0)}$ is given by

$$
\begin{gathered}
{ }^{t} \mathbf{n}_{\partial \Omega_{\alpha \beta}}\left(\mathbf{x}_{t}^{(0)}, t, \lambda\right)=\nabla \varphi_{\alpha \beta}\left(\mathbf{x}_{t}^{(0)}, t, \lambda\right) \\
=\left.\left(\frac{\partial \varphi_{\alpha \beta}}{\partial x_{1}^{(0)}}, \frac{\partial \varphi_{\alpha \beta}}{\partial x_{2}^{(0)}}, \ldots, \frac{\partial \varphi_{\alpha \beta}}{\partial x_{n}^{(0)}}\right)^{\mathrm{T}}\right|_{\left(t, \mathbf{x}_{t}^{(0)}\right)},
\end{gathered}
$$

and $t_{ \pm}=t \pm 0$.

If the flow $\mathbf{x}_{t}^{(i)}(i \in\{\alpha, \beta\})$ contacts with the boundary at time $t_{m}$, that is, $\mathbf{x}_{t_{m}}^{(i)}=\mathbf{x}_{m}=\mathbf{x}_{t_{m}}^{(0)}$, and the boundary $\partial \Omega_{\alpha \beta}$ is linear, independent of time $t$, we can obtain the 0thorder $G$-functions and the 1 st-order $G$-functions by simple computation

$$
\begin{aligned}
& G_{\partial \Omega_{\alpha \beta}}^{(0, i)}\left(\mathbf{x}_{m}, t_{m \pm}, \mathbf{P}_{i}, \lambda\right)=G_{\partial \Omega_{\alpha \beta}}^{(0, i)}\left(\mathbf{x}_{t_{m}}, t_{m \pm}, \mathbf{x}_{t_{m \pm}}^{(i)}, \mathbf{P}_{i}, \lambda\right) \\
& \quad=\left.{ }^{t} \mathbf{n}_{\partial \Omega_{\alpha \beta}}^{\mathrm{T}} \cdot \mathbf{F}^{(i)}\left(\mathbf{x}_{t}^{(i)}, t, \mathbf{P}_{i}\right)\right|_{\left(\mathbf{x}_{m}^{(0)}, \mathbf{x}_{m \pm}^{(i)}, t_{m \pm}\right)}, \\
& G_{\partial \Omega_{\alpha \beta}}^{(1, i)}\left(\mathbf{x}_{m}, t_{m \pm}, \mathbf{P}_{i}, \lambda\right)=G_{\partial \Omega_{\alpha \beta}}^{(1, i)}\left(\mathbf{x}_{t_{m}}, t_{m \pm}, \mathbf{x}_{t_{m \pm}}^{(i)}, \mathbf{P}_{i}, \lambda\right) \\
& \quad=\left.{ }^{t} \mathbf{n}_{\partial \Omega_{\alpha \beta}}^{\mathrm{T}} \cdot D_{\mathbf{x}_{t}(i)} \mathbf{F}^{(i)}\left(\mathbf{x}_{t}^{(i)}, t, \mathbf{P}_{i}\right)\right|_{\left(\mathbf{x}_{m}^{(0)}, \mathbf{x}_{m \pm}^{(i)}, t_{m \pm}\right)} .
\end{aligned}
$$

Here $t_{m \pm}=t_{m} \pm 0$ is to show the motion in different domains rather than on the boundaries, and $t_{m-}, t_{m+}$ are the time before approaching and after departing the corresponding boundary, respectively. In this case, the $G$-functions are simplified as $G_{\partial \Omega_{\alpha \beta}}^{(0, i)}\left(\mathbf{x}_{m}, t_{m \pm}\right)$ and $G_{\partial \Omega_{\alpha \beta}}^{(1, i)}\left(\mathbf{x}_{m}, t_{m \pm}\right)$.
For a discontinuous dynamical system in (22), there is a point $\mathbf{x}^{(0)}\left(t_{m}\right) \equiv \mathbf{x}_{m} \in \partial \Omega_{\alpha \beta}$ at time $t_{m}$ between two adjacent domains $\Omega_{i}(i \in\{\alpha, \beta\})$. For an arbitrarily small $\varepsilon>0$, there are two time intervals $\left[t_{m-\varepsilon}, t_{m}\right)$ and $\left(t_{m}, t_{m+\varepsilon}\right]$. Suppose $\mathbf{x}^{(i)}\left(t_{m-}\right)=\mathbf{x}_{m}=\mathbf{x}^{(j)}\left(t_{m+}\right)(i, j \in\{\alpha, \beta\})$. Both flows $\mathbf{x}^{(i)}(t)$ and $\mathbf{x}^{(j)}(t)$ are $C_{\left[t_{m-\varepsilon}, t_{m}\right)}^{r_{i}}-$ and $C_{\left(t_{m}, t_{m+\varepsilon}\right]}^{r_{j}}$-continuous for time $t(i, j \in\{\alpha, \beta\})$, and $\left\|d^{r_{i+1}} \mathbf{x}^{(i)} / d t^{r_{i}+1}\right\|<\infty\left(r_{i} \geq 1, i \in\{\alpha, \beta\}\right)$.

Based on the $G$-functions, the decision theorems of semipassable flow, sink flow, tangential flow, sliding bifurcation, and sliding fragmentation bifurcation to the separation boundary are stated in the form of lemma as follows.

Lemma 3 (see [42]). The flows $\mathbf{x}^{(\alpha)}(t)$ and $\mathbf{x}^{(\beta)}(t)$ at point $\left(\mathbf{x}_{m}, t_{m}\right)$ on the boundary $\partial \Omega_{\alpha \beta}$ are semipassable from domain $\Omega_{\alpha}$ to domain $\Omega_{\beta}$ if and only if

$$
\begin{array}{r}
\text { either } \quad G_{\partial \Omega_{\alpha \beta}}^{(\alpha)}\left(\mathbf{x}_{m}, t_{m-}, \mathbf{P}_{\alpha}, \lambda\right)>0, \\
\quad G_{\partial \Omega_{\alpha \beta}}^{(\beta)}\left(\mathbf{x}_{m}, t_{m+}, \mathbf{P}_{\beta}, \lambda\right)>0, \\
\quad \text { for } \mathbf{n}_{\partial \Omega_{\alpha \beta}} \longrightarrow \Omega_{\beta}, \\
\quad \text { or } \quad G_{\partial \Omega_{\alpha \beta}}^{(\alpha)}\left(\mathbf{x}_{m}, t_{m-}, \mathbf{P}_{\alpha}, \lambda\right)<0, \\
G_{\partial \Omega_{\alpha \beta}}^{(\beta)}\left(\mathbf{x}_{m}, t_{m+}, \mathbf{P}_{\beta}, \lambda\right)<0, \\
\quad \text { for } \mathbf{n}_{\partial \Omega_{\alpha \beta}} \longrightarrow \Omega_{\alpha} .
\end{array}
$$

Lemma 4 (see [42]). The flows $\mathbf{x}^{(\alpha)}(t)$ and $\mathbf{x}^{(\beta)}(t)$ at point $\left(\mathbf{x}_{m}, t_{m}\right)$ on the boundary $\partial \Omega_{\alpha \beta}$ are sink flows if and only if

$$
\begin{gathered}
\text { either } \quad G_{\partial \Omega_{\alpha \beta}}^{(\alpha)}\left(\mathbf{x}_{m}, t_{m-}, \mathbf{P}_{\alpha}, \lambda\right)>0, \\
\quad G_{\partial \Omega_{\alpha \beta}}^{(\beta)}\left(\mathbf{x}_{m}, t_{m-}, \mathbf{P}_{\beta}, \lambda\right)<0, \\
\text { or } \quad G_{\partial \Omega_{\alpha \beta}}^{(\alpha)}\left(\mathbf{x}_{m}, t_{m-}, \mathbf{P}_{\alpha}, \lambda\right)<0, \\
G_{\partial \Omega_{\alpha \beta}}^{(\beta)}\left(\mathbf{x}_{m}, t_{m-}, \mathbf{P}_{\beta}, \lambda\right)>0,
\end{gathered}
$$

$$
\text { for } \mathbf{n}_{\partial \Omega_{\alpha \beta}} \longrightarrow \Omega_{\alpha} \text {. }
$$

Lemma 5 (see [42]). For an arbitrarily small $\varepsilon>0$, there is a time interval $\left[t_{m-\varepsilon}, t_{m+\varepsilon}\right]$. Suppose $\mathbf{x}^{(i)}\left(t_{m \pm}\right)=$ $\mathbf{x}_{m}$. The flow $\mathbf{x}^{(i)}(t)$ is $C_{\left[t_{m-\varepsilon}, t_{m+\varepsilon}\right]}^{r_{i}}$-continuous for time $t$, and $\left\|d^{r_{i+1}} \mathbf{x}^{(i)} / d t^{r_{i}+1}\right\|<\infty\left(r_{i} \geq 2, i \in\{\alpha, \beta\}\right)$. A flow $\mathbf{x}^{(i)}(t)$ in $\Omega_{i}$ is tangential to the boundary $\partial \Omega_{\alpha \beta}$ if and only if

$$
\begin{gathered}
G_{\partial \Omega_{\alpha \beta}}^{(0, i)}\left(\mathbf{x}_{m}, t_{m}, \mathbf{P}_{i}, \lambda\right)=0, \\
\text { either } G_{\partial \Omega_{\alpha \beta}}^{(1, i)}\left(\mathbf{x}_{m}, t_{m}, \mathbf{P}_{i}, \lambda\right)<0 \quad \text { for } \mathbf{n}_{\partial \Omega_{\alpha \beta}} \longrightarrow \Omega_{j}, \\
\text { or } \quad G_{\partial \Omega_{\alpha \beta}}^{(1, i)}\left(\mathbf{x}_{m}, t_{m}, \mathbf{P}_{i}, \lambda\right)>0 \quad \text { for } \mathbf{n}_{\partial \Omega_{\alpha \beta}} \longrightarrow \Omega_{i},
\end{gathered}
$$

where $i, j \in\{\alpha, \beta\}, i \neq j$. 
Lemma 6 (see [42]). For an arbitrarily small $\varepsilon>0$, there are two time intervals $\left[t_{m-\varepsilon}, t_{m}\right)$ and $\left[t_{m-\varepsilon}, t_{m+\varepsilon}\right]$. Suppose $\mathbf{x}^{(\alpha)}\left(t_{m-}\right)=\mathbf{x}_{m}=\mathbf{x}^{(\beta)}\left(t_{m \pm}\right)$. The flows $\mathbf{x}^{(\alpha)}(t)$ and $\mathbf{x}^{(\beta)}(t)$ are $C_{\left[t_{m-\varepsilon}, t_{m}\right)}^{r_{\alpha}}$ - and $C_{\left[t_{m-\varepsilon}, t_{m+\varepsilon}\right]}^{r_{\beta}}$-continuous for time $t$ and $\left\|d^{r_{i+1}} \mathbf{x}^{(i)} / d t^{r_{i}+1}\right\|<\infty\left(r_{i} \geq 2\right.$ and $\left.i \in\{\alpha, \beta\}\right)$. The sliding bifurcation of the passable flows $\mathbf{x}^{(\alpha)}(t)$ and $\mathbf{x}^{(\beta)}(t)$ at point $\left(\mathbf{x}_{m}, t_{m}\right)$ switching to the nonpassable flow of the first kind on the boundary $\overrightarrow{\partial \Omega}_{\alpha \beta}$ occurs if and only if

$$
\begin{aligned}
& G_{\partial \Omega_{\alpha \beta}}^{(\beta)}\left(\mathbf{x}_{m}, t_{m \pm}, \mathbf{P}_{\beta}, \lambda\right)=0, \\
& G_{\partial \Omega_{\alpha \beta}}^{(\alpha)}\left(\mathbf{x}_{m}, t_{m-}, \mathbf{P}_{\alpha}, \lambda\right)>0 \text { for } \mathbf{n}_{\partial \Omega_{\alpha \beta}} \longrightarrow \Omega_{\beta}, \\
& G_{\partial \Omega_{\alpha \beta}}^{(\alpha)}\left(\mathbf{x}_{m}, t_{m-}, \mathbf{P}_{\alpha}, \lambda\right)<0 \text { for } \mathbf{n}_{\partial \Omega_{\alpha \beta}} \longrightarrow \Omega_{\alpha}, \\
& G_{\partial \Omega_{\alpha \beta}}^{(1, \beta)}\left(\mathbf{x}_{m}, t_{m \pm}, \mathbf{P}_{\beta}, \lambda\right)>0 \text { for } \mathbf{n}_{\partial \Omega_{\alpha \beta}} \longrightarrow \Omega_{\beta}, \\
& G_{\partial \Omega_{\alpha \beta}}^{(1, \beta)}\left(\mathbf{x}_{m}, t_{m \pm}, \mathbf{P}_{\beta}, \lambda\right)<0 \text { for } \mathbf{n}_{\partial \Omega_{\alpha \beta}} \longrightarrow \Omega_{\alpha} .
\end{aligned}
$$

Lemma 7 (see [42]). For an arbitrarily small $\varepsilon>0$, there are two time intervals $\left[t_{m-\varepsilon}, t_{m}\right)$ and $\left[t_{m-\varepsilon}, t_{m+\varepsilon}\right]$. Suppose $\mathbf{x}^{(\alpha)}\left(t_{m-}\right)=\mathbf{x}_{m}=\mathbf{x}^{(\beta)}\left(t_{m \pm}\right)$. The flows $\mathbf{x}^{(\alpha)}(t)$ and $\mathbf{x}^{(\beta)}(t)$ are $C_{\left[t_{m-\varepsilon}, t_{m}\right)}^{r_{\alpha}}$ - and $C_{\left[t_{m-\varepsilon}, t_{m+\varepsilon}\right]}^{r_{\beta}}$-continuous for time $t$ and $\left\|d^{r_{i+1}} \mathbf{x}^{(i)} / d t^{r_{i}+1}\right\|<\infty\left(r_{i} \geq 2\right.$ and $\left.i \in\{\alpha, \beta\}\right)$. The tangential bifurcation of the flows $\mathbf{x}^{(\alpha)}$ and $\mathbf{x}^{(\beta)}$ at point $\left(\mathbf{x}_{m}, t_{m}\right)$ on the boundary $\widetilde{\partial \Omega}_{\alpha \beta}$ is termed the sliding fragmentation bifurcation if and only if

$$
\begin{aligned}
& G_{\partial \Omega_{\alpha \beta}}^{(\beta)}\left(\mathbf{x}_{m}, t_{m \mp}, \mathbf{P}_{\beta}, \lambda\right)=0, \\
& G_{\partial \Omega_{\alpha \beta}}^{(\alpha)}\left(\mathbf{x}_{m}, t_{m-}, \mathbf{P}_{\alpha}, \lambda\right)>0 \text { for } \mathbf{n}_{\partial \Omega_{\alpha \beta}} \longrightarrow \Omega_{\beta}, \\
& G_{\partial \Omega_{\alpha \beta}}^{(\alpha)}\left(\mathbf{x}_{m}, t_{m-}, \mathbf{P}_{\alpha}, \lambda\right)<0 \text { for } \mathbf{n}_{\partial \Omega_{\alpha \beta}} \longrightarrow \Omega_{\alpha}, \\
& G_{\partial \Omega_{\alpha \beta}}^{(1, \beta)}\left(\mathbf{x}_{m}, t_{m \mp}, \mathbf{P}_{\beta}, \lambda\right)>0 \text { for } \mathbf{n}_{\partial \Omega_{\alpha \beta}} \longrightarrow \Omega_{\beta}, \\
& G_{\partial \Omega_{\alpha \beta}}^{(1, \beta)}\left(\mathbf{x}_{m}, t_{m \mp}, \mathbf{P}_{\beta}, \lambda\right)<0 \text { for } \mathbf{n}_{\partial \Omega_{\alpha \beta}} \longrightarrow \Omega_{\alpha} .
\end{aligned}
$$

More detailed theory on the flow switchability such as high-order $G$-functions, the definitions, or the decision theorems about various flow passability in discontinuous dynamical systems can be found in [42].

4.2. Analytical Conditions. According to the previous definitions and lemmas, the analytical switching conditions on discontinuous boundaries in the friction-induced oscillator with an elliptic control law and the friction force acting on the mass $M$ described in Section 3 will be developed in this subsection. From (12), the normal vectors of the absolute boundaries are given as

$$
\mathbf{n}_{\partial \Omega_{\alpha \beta}}=\mathbf{n}_{\partial \Omega_{\beta \alpha}}=\nabla \varphi_{\alpha \beta}=\left(\frac{\partial \varphi_{\alpha \beta}}{\partial x}, \frac{\partial \varphi_{\alpha \beta}}{\partial \dot{x}}\right)^{\mathrm{T}}=(0,1)^{\mathrm{T}},
$$

for $(\alpha, \beta) \in\{(2,3),(3,2),(1,4),(4,1)\}$, and

$$
\begin{aligned}
\mathbf{n}_{\partial \Omega_{\alpha \beta}} & =\mathbf{n}_{\partial \Omega_{\beta \alpha}}=\frac{a^{2} b^{2}}{2} \nabla \varphi_{\alpha \beta}=\frac{a^{2} b^{2}}{2}\left(\frac{\partial \varphi_{\alpha \beta}}{\partial x}, \frac{\partial \varphi_{\alpha \beta}}{\partial \dot{x}}\right)^{\mathrm{T}} \\
& =\left(b^{2} x, a^{2} y\right)^{\mathrm{T}}
\end{aligned}
$$

for $(\alpha, \beta) \in\{(1,2),(2,1),(3,4),(4,3)\}$, where $\nabla=$ $(\partial / \partial x, \partial / \partial y)^{\mathrm{T}}$ is a Hamilton operator.

Theorem 8. For the friction-induced oscillator with an elliptic control law and the friction force acting on the mass $M$ discussed in Section 2, there are the following results.

(i) The necessary and sufficient conditions for passable motion on the boundary $\mathbf{x}_{m} \equiv\left(x_{m}, y_{m}\right) \in \partial \Omega_{12}$ at time $t_{m}$ are

$$
\text { either } \begin{aligned}
b^{2} x_{m} y_{m}+a^{2} y_{m} F^{(1)}\left(\mathbf{x}_{m}, t_{m-}\right) & <0, \\
b^{2} x_{m} y_{m}+a^{2} y_{m} F^{(2)}\left(\mathbf{x}_{m}, t_{m+}\right) & <0, \\
& \text { for } \Omega_{1} \longrightarrow \Omega_{2}, \\
\text { or } \quad b^{2} x_{m} y_{m}+a^{2} y_{m} F^{(2)}\left(\mathbf{x}_{m}, t_{m-}\right) & >0, \\
b^{2} x_{m} y_{m}+a^{2} y_{m} F^{(1)}\left(\mathbf{x}_{m}, t_{m+}\right) & >0, \\
& \text { for } \Omega_{2} \longrightarrow \Omega_{1} .
\end{aligned}
$$

(ii) The necessary and sufficient conditions for passable motion on the boundary $\mathbf{x}_{m} \equiv\left(x_{m}, y_{m}\right) \in \partial \Omega_{23}$ at time $t_{m}$ are

$$
\text { either } \begin{aligned}
& F^{(2)}\left(\mathbf{x}_{m}, t_{m-}\right)<0, \\
& F^{(3)}\left(\mathbf{x}_{m}, t_{m+}\right)<0, \\
\text { or } \quad F^{(3)}\left(\mathbf{x}_{m}, t_{m-}\right) & >0, \\
F^{(2)}\left(\mathbf{x}_{m}, t_{m+}\right) & >0,
\end{aligned}
$$$$
\text { for } \Omega_{2} \longrightarrow \Omega_{3} \text {, }
$$

$$
\text { for } \Omega_{3} \longrightarrow \Omega_{2} \text {. }
$$

(iii) The necessary and sufficient conditions for passable motion on the boundary $\mathbf{x}_{m} \equiv\left(x_{m}, y_{m}\right) \in \partial \Omega_{34}$ at time $t_{m}$ are

$$
\text { either } \begin{aligned}
b^{2} x_{m} y_{m}+a^{2} y_{m} F^{(3)}\left(\mathbf{x}_{m}, t_{m-}\right) & >0, \\
b^{2} x_{m} y_{m}+a^{2} y_{m} F^{(4)}\left(\mathbf{x}_{m}, t_{m+}\right) & >0, \\
& \text { for } \Omega_{3} \longrightarrow \Omega_{4}, \\
\text { or } \quad b^{2} x_{m} y_{m}+a^{2} y_{m} F^{(4)}\left(\mathbf{x}_{m}, t_{m-}\right) & <0, \\
b^{2} x_{m} y_{m}+a^{2} y_{m} F^{(3)}\left(\mathbf{x}_{m}, t_{m+}\right) & <0, \\
& \text { for } \Omega_{4} \longrightarrow \Omega_{3} .
\end{aligned}
$$


(iv) The necessary and sufficient conditions for passable motion on the boundary $\mathbf{x}_{m} \equiv\left(x_{m}, y_{m}\right) \in \partial \Omega_{14}$ at time $t_{m}$ are

$$
\text { either } \begin{aligned}
F^{(1)}\left(\mathbf{x}_{m}, t_{m-}\right) & <0, \\
F^{(4)}\left(\mathbf{x}_{m}, t_{m+}\right) & <0, \\
\text { or } \quad F^{(4)}\left(\mathbf{x}_{m}, t_{m-}\right) & >0, \\
F^{(1)}\left(\mathbf{x}_{m}, t_{m+}\right) & >0,
\end{aligned}
$$$$
\text { for } \Omega_{1} \longrightarrow \Omega_{4} \text {, }
$$

$$
\text { for } \Omega_{4} \rightarrow \Omega_{1} \text {. }
$$

Proof. The mass $M$ moves along the conveyor belt with nonzero relative velocity before time $t_{m}$, and the relative velocity becomes zero at $t_{m}$; then after $t_{m}$ the relative velocity changes its direction. From the flow switchability theory on the discontinuous dynamical systems, the flow in domain $\Omega_{\lambda}(\lambda \in\{\alpha, \beta\})$ or $\Omega_{\gamma}(\gamma \in\{i, j\})$ passes through the boundary $\partial \Omega_{\alpha \beta}(\alpha \beta \in\{12,21,34,43\})$ or $\partial \Omega_{i j}(i j \in$ $\{23,32,14,41\})$ into domain $\Omega_{\bar{\lambda}}(\bar{\lambda} \in\{\alpha, \beta\}, \bar{\lambda} \neq \lambda)$ or $\Omega_{\bar{\gamma}}(\bar{\gamma} \in\{i, j\}, \bar{\gamma} \neq \gamma)$, which can be expressed by Lemma 3 . So 0 th-order $G$-functions on such boundaries are needed.

According to (27), the 0th-order $G$-functions on the boundary $\partial \Omega_{12}$ are

$$
\begin{aligned}
& G_{\partial \Omega_{12}}^{(0,1)}\left(\mathbf{x}_{m}, t_{m \pm}\right)=\mathbf{n}_{\partial \Omega_{12}}^{\mathrm{T}} \cdot \mathbf{F}^{(1)}\left(\mathbf{x}_{m}, t_{m \pm}\right), \\
& G_{\partial \Omega_{12}}^{(0,2)}\left(\mathbf{x}_{m}, t_{m \pm}\right)=\mathbf{n}_{\partial \Omega_{12}}^{\mathrm{T}} \cdot \mathbf{F}^{(2)}\left(\mathbf{x}_{m}, t_{m \pm}\right) .
\end{aligned}
$$

From (13) and (35), (44) can be computed as

$$
\begin{aligned}
& G_{\partial \Omega_{12}}^{(0,1)}\left(\mathbf{x}_{m}, t_{m-}\right)=b^{2} x_{m} y_{m}+a^{2} y_{m} F^{(1)}\left(\mathbf{x}_{m}, t_{m-}\right), \\
& G_{\partial \Omega_{12}}^{(0,2)}\left(\mathbf{x}_{m}, t_{m+}\right)=b^{2} x_{m} y_{m}+a^{2} y_{m} F^{(2)}\left(\mathbf{x}_{m}, t_{m+}\right) .
\end{aligned}
$$

By Lemma 3, the passable motion on the boundary $\mathbf{x}_{m} \in \partial \Omega_{12}$ at time $t_{m}$ appears if and only if

$$
\begin{aligned}
& G_{\partial \Omega_{12}}^{(0,1)}\left(\mathbf{x}_{m}, t_{m-}\right)<0, \\
& G_{\partial \Omega_{12}}^{(0,2)}\left(\mathbf{x}_{m}, t_{m+}\right)<0,
\end{aligned}
$$

$$
\text { for } \Omega_{1} \longrightarrow \Omega_{2} \text {. }
$$

From (45) and (46), one obtains

$$
\begin{aligned}
& b^{2} x_{m} y_{m}+a^{2} y_{m} F^{(1)}\left(\mathbf{x}_{m}, t_{m-}\right)<0, \\
& b^{2} x_{m} y_{m}+a^{2} y_{m} F^{(2)}\left(\mathbf{x}_{m}, t_{m+}\right)<0, \\
& \quad \text { for } \Omega_{1} \rightarrow \Omega_{2} .
\end{aligned}
$$

Thus, (36) in (i) holds, and (37) in (i) and (40) and (41) in (iii) can be proved similarly.

From (27), the 0th-order G-functions on the boundary $\partial \Omega_{23}$ are

$$
\begin{aligned}
& G_{\partial \Omega_{23}}^{(0,2)}\left(\mathbf{x}_{m}, t_{m \pm}\right)=\mathbf{n}_{\partial \Omega_{23}}^{\mathrm{T}} \cdot \mathbf{F}^{(2)}\left(\mathbf{x}_{m}, t_{m \pm}\right), \\
& G_{\partial \Omega_{23}}^{(0,3)}\left(\mathbf{x}_{m}, t_{m \pm}\right)=\mathbf{n}_{\partial \Omega_{23}}^{\mathrm{T}} \cdot \mathbf{F}^{(3)}\left(\mathbf{x}_{m}, t_{m \pm}\right) .
\end{aligned}
$$

From (13) and (34), (48) can be computed as

$$
\begin{aligned}
& G_{\partial \Omega_{23}}^{(0,2)}\left(\mathbf{x}_{m}, t_{m-}\right)=F^{(2)}\left(\mathbf{x}_{m}, t_{m-}\right), \\
& G_{\partial \Omega_{23}}^{(0,3)}\left(\mathbf{x}_{m}, t_{m+}\right)=F^{(3)}\left(\mathbf{x}_{m}, t_{m+}\right) .
\end{aligned}
$$

By Lemma 3, the passable motion on the boundary $\mathbf{x}_{m} \in \partial \Omega_{23}$ at time $t_{m}$ appears if and only if

$$
\begin{aligned}
& G_{\partial \Omega_{23}}^{(0,2)}\left(\mathbf{x}_{m}, t_{m-}\right)<0, \\
& G_{\partial \Omega_{23}}^{(0,3)}\left(\mathbf{x}_{m}, t_{m+}\right)<0,
\end{aligned}
$$

for $\Omega_{2} \longrightarrow \Omega_{3}$.

By (49) and (50), one obtains

$$
\begin{aligned}
& F^{(2)}\left(\mathbf{x}_{m}, t_{m-}\right)<0, \\
& F^{(3)}\left(\mathbf{x}_{m}, t_{m+}\right)<0, \\
& \quad \text { for } \Omega_{2} \longrightarrow \Omega_{3} .
\end{aligned}
$$

Thus, (38) in (ii) holds, and (39) in (ii) and (42) and (43) in (iv) can be proved similarly.

Theorem 9. For the friction-induced oscillator with an elliptic control law and the friction force acting on the mass $M$ discussed in Section 2, there are the following results.

(i) The necessary and sufficient conditions for sliding motion on the boundary $\mathbf{x}_{m} \equiv\left(x_{m}, y_{m}\right) \in \partial \Omega_{12}$ at time $t_{m}$ are

$$
\begin{aligned}
& b^{2} x_{m} y_{m}+a^{2} y_{m} F^{(1)}\left(\mathbf{x}_{m}, t_{m-}\right)<0, \\
& b^{2} x_{m} y_{m}+a^{2} y_{m} F^{(2)}\left(\mathbf{x}_{m}, t_{m-}\right)>0 .
\end{aligned}
$$

(ii) The necessary and sufficient conditions for stick motion on the boundary $\mathbf{x}_{m} \equiv\left(x_{m}, y_{m}\right) \in \partial \Omega_{23}$ at time $t_{m}$ are

$$
\begin{aligned}
& F^{(2)}\left(\mathbf{x}_{m}, t_{m-}\right)<0, \\
& F^{(3)}\left(\mathbf{x}_{m}, t_{m-}\right)>0 .
\end{aligned}
$$

(iii) The necessary and sufficient conditions for sliding motion on the boundary $\mathbf{x}_{m} \equiv\left(x_{m}, y_{m}\right) \in \partial \Omega_{34}$ at time $t_{m}$ are

$$
\begin{aligned}
& b^{2} x_{m} y_{m}+a^{2} y_{m} F^{(3)}\left(\mathbf{x}_{m}, t_{m-}\right)>0, \\
& b^{2} x_{m} y_{m}+a^{2} y_{m} F^{(4)}\left(\mathbf{x}_{m}, t_{m-}\right)<0 .
\end{aligned}
$$

(iv) The necessary and sufficient conditions for stick motion on the boundary $\mathbf{x}_{m} \equiv\left(x_{m}, y_{m}\right) \in \partial \Omega_{14}$ at time $t_{m}$ are

$$
\begin{aligned}
& F^{(1)}\left(\mathbf{x}_{m}, t_{m-}\right)<0, \\
& F^{(4)}\left(\mathbf{x}_{m}, t_{m-}\right)>0 .
\end{aligned}
$$

Proof. The displacement and velocity of the mass $M$ satisfy the elliptic control law; such motion is called the sliding 
motion. From the flow switchability theory on the discontinuous dynamical systems, the sliding motion is that the flow in domain $\Omega_{\lambda}(\lambda \in\{\alpha, \beta\})$ reaches the boundary $\partial \Omega_{\alpha \beta}(\alpha \beta \epsilon$ $\{12,21,34,43\})$ and moves along the boundary $\partial \Omega_{\alpha \beta}(\alpha \beta \in$ $\{12,21,34,43\})$. Thus it can be predicted by Lemma 4 .

From (27), the 0th-order $G$-functions on the boundary $\partial \Omega_{12}$ are

$$
\begin{aligned}
& G_{\partial \Omega_{12}}^{(0,1)}\left(\mathbf{x}_{m}, t_{m \pm}\right)=\mathbf{n}_{\partial \Omega_{12}}^{\mathrm{T}} \cdot \mathbf{F}^{(1)}\left(\mathbf{x}_{m}, t_{m \pm}\right), \\
& G_{\partial \Omega_{12}}^{(0,2)}\left(\mathbf{x}_{m}, t_{m \pm}\right)=\mathbf{n}_{\partial \Omega_{12}}^{\mathrm{T}} \cdot \mathbf{F}^{(2)}\left(\mathbf{x}_{m}, t_{m \pm}\right) .
\end{aligned}
$$

From (13) and (35), (56) can be computed as

$$
\begin{aligned}
& G_{\partial \Omega_{12}}^{(0,1)}\left(\mathbf{x}_{m}, t_{m-}\right)=b^{2} x_{m} y_{m}+a^{2} y_{m} F^{(1)}\left(\mathbf{x}_{m}, t_{m-}\right), \\
& G_{\partial \Omega_{12}}^{(0,2)}\left(\mathbf{x}_{m}, t_{m-}\right)=b^{2} x_{m} y_{m}+a^{2} y_{m} F^{(2)}\left(\mathbf{x}_{m}, t_{m-}\right) .
\end{aligned}
$$

By Lemma 4 , the sliding motion on the boundary $\mathbf{x}_{m} \in \partial \Omega_{12}$ at time $t_{m}$ appears if and only if

$$
\begin{aligned}
& G_{\partial \Omega_{12}}^{(0,1)}\left(\mathbf{x}_{m}, t_{m-}\right)<0, \\
& G_{\partial \Omega_{12}}^{(0,2)}\left(\mathbf{x}_{m}, t_{m-}\right)>0 .
\end{aligned}
$$

From (57) and (58), one obtains

$$
\begin{aligned}
& b^{2} x_{m} y_{m}+a^{2} y_{m} F^{(1)}\left(\mathbf{x}_{m}, t_{m-}\right)<0, \\
& b^{2} x_{m} y_{m}+a^{2} y_{m} F^{(2)}\left(\mathbf{x}_{m}, t_{m-}\right)>0 .
\end{aligned}
$$

Thus, (i) holds, and (iii) can be proved similarly.

The mass $M$ moves together with the conveyor belt and the displacement and velocity of the mass $M$ do not satisfy the elliptic control law; such motion is called the stick motion. From the flow switchability theory on the discontinuous dynamical systems, the stick motion is that the flow in domain $\Omega_{\gamma}(\gamma \in\{i, j\})$ reaches the boundary $\partial \Omega_{i j}(i j \in$ $\{23,32,14,41\})$ and moves along the boundary $\partial \Omega_{i j}(i j \in$ $\{23,32,14,41\})$. Thus it can be predicted by Lemma 4 .

By (27), the 0th-order $G$-functions on the boundary $\partial \Omega_{23}$ are

$$
\begin{aligned}
& G_{\partial \Omega_{23}}^{(0,2)}\left(\mathbf{x}_{m}, t_{m \pm}\right)=\mathbf{n}_{\partial \Omega_{23}}^{\mathrm{T}} \cdot \mathbf{F}^{(2)}\left(\mathbf{x}_{m}, t_{m \pm}\right), \\
& G_{\partial \Omega_{23}}^{(0,3)}\left(\mathbf{x}_{m}, t_{m \pm}\right)=\mathbf{n}_{\partial \Omega_{23}}^{\mathrm{T}} \cdot \mathbf{F}^{(3)}\left(\mathbf{x}_{m}, t_{m \pm}\right) .
\end{aligned}
$$

From (13) and (34), (60) can be computed as

$$
\begin{aligned}
& G_{\partial \Omega_{23}}^{(0,2)}\left(\mathbf{x}_{m}, t_{m-}\right)=F^{(2)}\left(\mathbf{x}_{m}, t_{m-}\right), \\
& G_{\partial \Omega_{23}}^{(0,3)}\left(\mathbf{x}_{m}, t_{m-}\right)=F^{(3)}\left(\mathbf{x}_{m}, t_{m-}\right) .
\end{aligned}
$$

By Lemma 4, the stick motion on the boundary $\mathbf{x}_{m} \in \partial \Omega_{23}$ at time $t_{m}$ appears if and only if

$$
\begin{aligned}
& G_{\partial \Omega_{23}}^{(0,2)}\left(\mathbf{x}_{m}, t_{m-}\right)<0, \\
& G_{\partial \Omega_{23}}^{(0,3)}\left(\mathbf{x}_{m}, t_{m-}\right)>0 .
\end{aligned}
$$

From (61) and (62), one obtains

$$
\begin{aligned}
& F^{(2)}\left(\mathbf{x}_{m}, t_{m-}\right)<0, \\
& F^{(3)}\left(\mathbf{x}_{m}, t_{m-}\right)>0 .
\end{aligned}
$$

Thus, (ii) holds, and (iv) can be proved similarly.

Theorem 10. For the friction-induced oscillator with an elliptic control law and the friction force acting on the mass $M$ discussed in Section 2, there are the following results.

(i) The necessary and sufficient conditions for grazing motion on the boundary $\mathbf{x}_{m} \equiv\left(x_{m}, y_{m}\right) \in \partial \Omega_{12}$ in domain $\Omega_{1}$ or domain $\Omega_{2}$ at time $t_{m}$ are

$$
\begin{array}{r}
b^{2} x_{m} y_{m}+a^{2} y_{m} F^{(1)}\left(\mathbf{x}_{m}, t_{m \pm}\right)=0, \\
b^{2} x_{m} F^{(1)}\left(\mathbf{x}_{m}, t_{m \pm}\right)+a^{2} y_{m} D F^{(1)}\left(\mathbf{x}_{m}, t_{m \pm}\right)>0,
\end{array}
$$

$$
\text { in } \Omega_{1} \text { for } \partial \Omega_{12} \text {, }
$$

or

$$
\begin{aligned}
& b^{2} x_{m} y_{m}+a^{2} y_{m} F^{(2)}\left(\mathbf{x}_{m}, t_{m \pm}\right)=0, \\
& b^{2} x_{m} F^{(2)}\left(\mathbf{x}_{m}, t_{m \pm}\right)+a^{2} y_{m} D F^{(2)}\left(\mathbf{x}_{m}, t_{m \pm}\right)<0, \\
& \text { in } \Omega_{2} \text { for } \partial \Omega_{12} .
\end{aligned}
$$

(ii) The necessary and sufficient conditions for grazing motion on the boundary $\mathbf{x}_{m} \equiv\left(x_{m}, y_{m}\right) \in \partial \Omega_{34}$ in domain $\Omega_{3}$ or domain $\Omega_{4}$ at time $t_{m}$ are

$$
\begin{array}{r}
b^{2} x_{m} y_{m}+a^{2} y_{m} F^{(3)}\left(\mathbf{x}_{m}, t_{m \pm}\right)=0, \\
b^{2} x_{m} F^{(3)}\left(\mathbf{x}_{m}, t_{m \pm}\right)+a^{2} y_{m} D F^{(3)}\left(\mathbf{x}_{m}, t_{m \pm}\right)<0, \\
\text { in } \Omega_{3} \text { for } \partial \Omega_{34},
\end{array}
$$

or

$$
\begin{aligned}
& b^{2} x_{m} y_{m}+a^{2} y_{m} F^{(4)}\left(\mathbf{x}_{m}, t_{m \pm}\right)=0 \\
& b^{2} x_{m} F^{(4)}\left(\mathbf{x}_{m}, t_{m \pm}\right)+a^{2} y_{m} D F^{(4)}\left(\mathbf{x}_{m}, t_{m \pm}\right)>0 \\
& \text { in } \Omega_{4} \text { for } \partial \Omega_{34} .
\end{aligned}
$$

(iii) The necessary and sufficient conditions for grazing motion on the boundary $\mathbf{x}_{m} \equiv\left(x_{m}, y_{m}\right) \in \partial \Omega_{14}$ in domain $\Omega_{1}$ or domain $\Omega_{4}$ at time $t_{m}$ are

$$
\begin{aligned}
F^{(1)}\left(\mathbf{x}_{m}, t_{m \pm}\right) & =0, \\
D F^{(1)}\left(\mathbf{x}_{m}, t_{m \pm}\right) & >0,
\end{aligned}
$$

$$
\text { in } \Omega_{1} \text { for } \partial \Omega_{14} \text {, }
$$

or

$$
\begin{aligned}
F^{(4)}\left(\mathbf{x}_{m}, t_{m \pm}\right) & =0, \\
D F^{(4)}\left(\mathbf{x}_{m}, t_{m \pm}\right) & <0,
\end{aligned}
$$


(iv) The necessary and sufficient conditions for grazing motion on the boundary $\mathbf{x}_{m} \equiv\left(x_{m}, y_{m}\right) \in \partial \Omega_{23}$ in domain $\Omega_{2}$ or domain $\Omega_{3}$ at time $t_{m}$ are

$$
\begin{aligned}
F^{(2)}\left(\mathbf{x}_{m}, t_{m \pm}\right) & =0, \\
D F^{(2)}\left(\mathbf{x}_{m}, t_{m \pm}\right) & >0,
\end{aligned}
$$

$$
\text { in } \Omega_{2} \text { for } \partial \Omega_{23} \text {, }
$$

or

$$
\begin{aligned}
F^{(3)}\left(\mathbf{x}_{m}, t_{m \pm}\right) & =0, \\
D F^{(3)}\left(\mathbf{x}_{m}, t_{m \pm}\right) & <0,
\end{aligned}
$$

$$
\text { in } \Omega_{3} \text { for } \partial \Omega_{23} \text {. }
$$

Proof. When the mass $M$ is moving along the conveyor belt with nonzero relative velocity, the relative velocity equals zero at time $t_{m}$ and then it restores to the original relationship. From the flow switchability theory on the discontinuous dynamical systems, the flow of the motion in domain $\Omega_{\lambda}(\lambda \in$ $\{\alpha, \beta\})$ or $\Omega_{\gamma}(\gamma \in\{i, j\})$ reaches the boundary $\partial \Omega_{\alpha \beta}(\alpha \beta \in$ $\{12,21,34,43\})$ or $\partial \Omega_{i j}(i j \in\{23,32,14,41\})$ and then returns to domain $\Omega_{\lambda}(\lambda \in\{\alpha, \beta\})$ or $\Omega_{\gamma}(\gamma \in\{i, j\})$. Such motion is called grazing motion on the boundary $\partial \Omega_{\alpha \beta}(\alpha \beta \in\{12,21,34,43\})$ or $\partial \Omega_{i j}(i j \in\{23,32,14,41\})$. So the analytical conditions of grazing motion on boundary $\partial \Omega_{\alpha \beta}(\alpha \beta \in\{12,21,34,43\})$ or $\partial \Omega_{i j}(i j \in\{23,32,14,41\})$ can be obtained by Lemma 5 . Thus the 0 th-order $G$-functions and the 1st-order $G$-functions on such boundaries are needed.

According to (27) and (28), the 0th-order $G$-functions and the 1st-order $G$-functions on the boundary $\partial \Omega_{12}$ in domain $\Omega_{1}$ are

$$
\begin{aligned}
& G_{\partial \Omega_{12}}^{(0,1)}\left(\mathbf{x}_{m}, t_{m \pm}\right)=\mathbf{n}_{\partial \Omega_{12}}^{\mathrm{T}} \cdot \mathbf{F}^{(1)}\left(\mathbf{x}_{m}, t_{m \pm}\right), \\
& G_{\partial \Omega_{12}}^{(1,1)}\left(\mathbf{x}_{m}, t_{m \pm}\right)=\mathbf{n}_{\partial \Omega_{12}}^{\mathrm{T}} \cdot D \mathbf{F}^{(1)}\left(\mathbf{x}_{m}, t_{m \pm}\right) .
\end{aligned}
$$

From (13) and (35), (72) can be computed as

$$
\begin{aligned}
G_{\partial \Omega_{12}}^{(0,1)}\left(\mathbf{x}_{m}, t_{m \pm}\right)= & b^{2} x_{m} y_{m}+a^{2} y_{m} F^{(1)}\left(\mathbf{x}_{m}, t_{m \pm}\right), \\
G_{\partial \Omega_{12}}^{(1,1)}\left(\mathbf{x}_{m}, t_{m \pm}\right)= & b^{2} x_{m} F^{(1)}\left(\mathbf{x}_{m}, t_{m \pm}\right) \\
& +a^{2} y_{m} D F^{(1)}\left(\mathbf{x}_{m}, t_{m \pm}\right) .
\end{aligned}
$$

By Lemma 5, the grazing motion on the boundary $\mathbf{x}_{m} \equiv$ $\left(x_{m}, y_{m}\right) \in \partial \Omega_{12}$ in domain $\Omega_{1}$ appears if and only if

$$
\begin{aligned}
& G_{\partial \Omega_{12}}^{(0,1)}\left(\mathbf{x}_{m}, t_{m \pm}\right)=0, \\
& G_{\partial \Omega_{12}}^{(1,1)}\left(\mathbf{x}_{m}, t_{m \pm}\right)>0 .
\end{aligned}
$$

From (73) and (74), one obtains

$$
\begin{array}{r}
b^{2} x_{m} y_{m}+a^{2} y_{m} F^{(1)}\left(\mathbf{x}_{m}, t_{m \pm}\right)=0 \\
b^{2} x_{m} F^{(1)}\left(\mathbf{x}_{m}, t_{m \pm}\right)+a^{2} y_{m} D F^{(1)}\left(\mathbf{x}_{m}, t_{m \pm}\right)>0 \\
\text { in } \Omega_{1} \text { for } \partial \Omega_{12} .
\end{array}
$$

Thus (64) in (i) holds, and (65) in (i) and (66) and (67) in (ii) can be proved similarly.

By (27) and (28), the 0th-order G-functions and the 1storder $G$-functions on the boundary $\partial \Omega_{14}$ in domain $\Omega_{1}$ are

$$
\begin{aligned}
& G_{\partial \Omega_{14}}^{(0,1)}\left(\mathbf{x}_{m}, t_{m \pm}\right)=\mathbf{n}_{\partial \Omega_{14}}^{\mathrm{T}} \cdot \mathbf{F}^{(1)}\left(\mathbf{x}_{m}, t_{m \pm}\right), \\
& G_{\partial \Omega_{14}}^{(1,1)}\left(\mathbf{x}_{m}, t_{m \pm}\right)=\mathbf{n}_{\partial \Omega_{14}}^{\mathrm{T}} \cdot D \mathbf{F}^{(1)}\left(\mathbf{x}_{m}, t_{m \pm}\right) .
\end{aligned}
$$

From (13) and (34), (76) can be computed as

$$
\begin{aligned}
& G_{\partial \Omega_{14}}^{(0,1)}\left(\mathbf{x}_{m}, t_{m \pm}\right)=F^{(1)}\left(\mathbf{x}_{m}, t_{m \pm}\right) \\
& G_{\partial \Omega_{14}}^{(1,1)}\left(\mathbf{x}_{m}, t_{m \pm}\right)=D F^{(1)}\left(\mathbf{x}_{m}, t_{m \pm}\right) .
\end{aligned}
$$

By Lemma 5, the grazing motion on the boundary $\mathbf{x}_{m} \equiv$ $\left(x_{m}, y_{m}\right) \in \partial \Omega_{14}$ in domain $\Omega_{1}$ appears if and only if

$$
\begin{aligned}
& G_{\partial \Omega_{14}}^{(0,1)}\left(\mathbf{x}_{m}, t_{m \pm}\right)=0, \\
& G_{\partial \Omega_{14}}^{(1,1)}\left(\mathbf{x}_{m}, t_{m \pm}\right)>0 .
\end{aligned}
$$

From (77) and (78), one obtains

$$
\begin{aligned}
F^{(1)}\left(\mathbf{x}_{m}, t_{m \pm}\right) & =0, \\
D F^{(1)}\left(\mathbf{x}_{m}, t_{m \pm}\right) & >0,
\end{aligned}
$$

in $\Omega_{1}$ for $\partial \Omega_{14}$.

Thus (68) in (iii) holds, and (69) in (iii) and (70) and (71) in (iv) can be proved similarly.

Theorem 11. For the friction-induced oscillator with an elliptic control law and the friction force acting on the mass $M$ discussed in Section 2, there are the following results.

(i) The appearance conditions for the sliding motion of the mass $M$ at $\mathbf{x}_{m} \equiv\left(x_{m}, y_{m}\right) \in \partial \Omega_{12}$ are

$$
\begin{array}{r}
b^{2} x_{m} y_{m}+a^{2} y_{m} F^{(1)}\left(\mathbf{x}_{m}, t_{m-}\right)<0, \\
b^{2} x_{m} y_{m}+a^{2} y_{m} F^{(2)}\left(\mathbf{x}_{m}, t_{m \pm}\right)=0, \\
b^{2} x_{m} F^{(2)}\left(\mathbf{x}_{m}, t_{m \pm}\right)+a^{2} y_{m} D F^{(2)}\left(\mathbf{x}_{m}, t_{m \pm}\right)<0,
\end{array}
$$

$$
\text { for } \Omega_{1} \longrightarrow \partial \Omega_{12}
$$

or

$$
\begin{aligned}
b^{2} x_{m} y_{m}+a^{2} y_{m} F^{(1)}\left(\mathbf{x}_{m}, t_{m \pm}\right) & =0, \\
b^{2} x_{m} F^{(1)}\left(\mathbf{x}_{m}, t_{m \pm}\right)+a^{2} y_{m} D F^{(1)}\left(\mathbf{x}_{m}, t_{m \pm}\right) & >0, \\
b^{2} x_{m} y_{m}+a^{2} y_{m} F^{(2)}\left(\mathbf{x}_{m}, t_{m-}\right) & >0, \\
\text { for } \Omega_{2} & \longrightarrow \partial \Omega_{12} .
\end{aligned}
$$


(ii) The appearance conditions for the stick motion of the mass $M$ at $\mathbf{x}_{m} \equiv\left(x_{m}, y_{m}\right) \in \partial \Omega_{23}$ are

$$
\begin{aligned}
F^{(2)}\left(\mathbf{x}_{m}, t_{m-}\right) & <0, \\
F^{(3)}\left(\mathbf{x}_{m}, t_{m \pm}\right) & =0, \\
D F^{(3)}\left(\mathbf{x}_{m}, t_{m \pm}\right) & <0, \\
& \text { for } \Omega_{2} \longrightarrow \partial \Omega_{23},
\end{aligned}
$$

or

$$
\begin{aligned}
F^{(2)}\left(\mathbf{x}_{m}, t_{m \pm}\right) & =0, \\
D F^{(2)}\left(\mathbf{x}_{m}, t_{m \pm}\right) & >0, \\
F^{(3)}\left(\mathbf{x}_{m}, t_{m-}\right)>0, & \text { for } \Omega_{3} \longrightarrow \partial \Omega_{23} .
\end{aligned}
$$

(iii) The appearance conditions for the sliding motion of the mass $M$ at $\mathbf{x}_{m} \equiv\left(x_{m}, y_{m}\right) \in \partial \Omega_{34}$ are

$$
\begin{aligned}
b^{2} x_{m} y_{m}+a^{2} y_{m} F^{(3)}\left(\mathbf{x}_{m}, t_{m-}\right) & >0, \\
b^{2} x_{m} y_{m}+a^{2} y_{m} F^{(4)}\left(\mathbf{x}_{m}, t_{m \pm}\right) & =0, \\
b^{2} x_{m} F^{(4)}\left(\mathbf{x}_{m}, t_{m \pm}\right)+a^{2} y_{m} D F^{(4)}\left(\mathbf{x}_{m}, t_{m \pm}\right) & >0, \\
\text { for } \Omega_{3} & \longrightarrow \partial \Omega_{34},
\end{aligned}
$$

or

$$
\begin{aligned}
b^{2} x_{m} y_{m}+a^{2} y_{m} F^{(3)}\left(\mathbf{x}_{m}, t_{m \pm}\right) & =0, \\
b^{2} x_{m} F^{(3)}\left(\mathbf{x}_{m}, t_{m \pm}\right)+a^{2} y_{m} D F^{(3)}\left(\mathbf{x}_{m}, t_{m \pm}\right) & <0, \\
b^{2} x_{m} y_{m}+a^{2} y_{m} F^{(4)}\left(\mathbf{x}_{m}, t_{m-}\right) & <0, \\
\text { for } \Omega_{4} & \longrightarrow \partial \Omega_{34} .
\end{aligned}
$$

(iv) The appearance conditions for the stick motion of the mass $M$ at $\mathbf{x}_{m} \equiv\left(x_{m}, y_{m}\right) \in \partial \Omega_{14}$ are

$$
\begin{aligned}
F^{(1)}\left(\mathbf{x}_{m}, t_{m-}\right) & <0, \\
F^{(4)}\left(\mathbf{x}_{m}, t_{m \pm}\right) & =0, \\
D F^{(4)}\left(\mathbf{x}_{m}, t_{m \pm}\right) & <0, \\
& \text { for } \Omega_{1} \longrightarrow \partial \Omega_{14},
\end{aligned}
$$

or

$$
\begin{gathered}
F^{(1)}\left(\mathbf{x}_{m}, t_{m \pm}\right)=0, \\
D F^{(1)}\left(\mathbf{x}_{m}, t_{m \pm}\right)>0, \\
F^{(4)}\left(\mathbf{x}_{m}, t_{m-}\right)>0,
\end{gathered}
$$

Proof. When a flow in a domain reaches the sliding or stick boundary at time $t_{m}$ and then the sliding or stick motion occurs, such time $t_{m}$ may not be the onset time of the sliding or stick motion on this boundary. When conditions (36), (37), (40), (41) or (38), (39), (42), and (43) cannot be satisfied, the passable flow vanishes and the sliding or stick motion will begin at time $t_{m}$. From the flow switchability theory on the discontinuous dynamical systems, time $t_{m}$ is the switching time from semipassable flow to nonpassable flow. Thus the analytical conditions of the appearance of the sliding or stick motion on the boundary $\partial \Omega_{\alpha \beta}(\alpha \beta \in\{12,21,34,43\})$ or $\partial \Omega_{i j}(i j \in\{23,32,14,41\})$ can be obtained by Lemma 6 . Therefore, the 0 th-order $G$-functions and the 1st-order $G$ functions on such boundaries are needed.

From (27) and (28), the 0th-order $G$-functions and the 1st-order $G$-functions on the boundary $\partial \Omega_{12}$ in domain $\Omega_{1}$ and domain $\Omega_{2}$ are

$$
\begin{aligned}
G_{\partial \Omega_{12}}^{(0,1)}\left(\mathbf{x}_{m}, t_{m \pm}\right) & =\mathbf{n}_{\partial \Omega_{12}}^{\mathrm{T}} \cdot \mathbf{F}^{(1)}\left(\mathbf{x}_{m}, t_{m \pm}\right), \\
G_{\partial \Omega_{12}}^{(1,1)}\left(\mathbf{x}_{m}, t_{m \pm}\right) & =\mathbf{n}_{\partial \Omega_{12}}^{\mathrm{T}} \cdot D \mathbf{F}^{(1)}\left(\mathbf{x}_{m}, t_{m \pm}\right), \\
& \text { in } \Omega_{1} \text { for } \partial \Omega_{12}, \\
G_{\partial \Omega_{12}}^{(0,2)}\left(\mathbf{x}_{m}, t_{m \pm}\right) & =\mathbf{n}_{\partial \Omega_{12}}^{\mathrm{T}} \cdot \mathbf{F}^{(2)}\left(\mathbf{x}_{m}, t_{m \pm}\right), \\
G_{\partial \Omega_{12}}^{(1,2)}\left(\mathbf{x}_{m}, t_{m \pm}\right) & =\mathbf{n}_{\partial \Omega_{12}}^{\mathrm{T}} \cdot D \mathbf{F}^{(2)}\left(\mathbf{x}_{m}, t_{m \pm}\right), \\
& \text { in } \Omega_{2} \text { for } \partial \Omega_{12} .
\end{aligned}
$$

From (13) and (35), (88) can be computed as

$$
\begin{aligned}
& G_{\partial \Omega_{12}}^{(0,1)}\left(\mathbf{x}_{m}, t_{m \pm}\right)= b^{2} x_{m} y_{m}+a^{2} y_{m} F^{(1)}\left(\mathbf{x}_{m}, t_{m \pm}\right), \\
& G_{\partial \Omega_{12}}^{(1,1)}\left(\mathbf{x}_{m}, t_{m \pm}\right)= b^{2} x_{m} F^{(1)}\left(\mathbf{x}_{m}, t_{m \pm}\right) \\
&+a^{2} y_{m} D F^{(1)}\left(\mathbf{x}_{m}, t_{m \pm}\right), \\
& \text { in } \Omega_{1} \text { for } \partial \Omega_{12}, \\
& G_{\partial \Omega_{12}}^{(0,2)}\left(\mathbf{x}_{m}, t_{m \pm}\right)= b^{2} x_{m} y_{m}+a^{2} y_{m} F^{(2)}\left(\mathbf{x}_{m}, t_{m \pm}\right), \\
& G_{\partial \Omega_{12}}^{(1,2)}\left(\mathbf{x}_{m}, t_{m \pm}\right)= b^{2} x_{m} F^{(2)}\left(\mathbf{x}_{m}, t_{m \pm}\right) \\
&+a^{2} y_{m} D F^{(2)}\left(\mathbf{x}_{m}, t_{m \pm}\right), \\
& \text { in } \Omega_{2} \text { for } \partial \Omega_{12} .
\end{aligned}
$$

By Lemma 6, the appearance conditions for the sliding motion of the mass $M$ at $\mathbf{x}_{m} \equiv\left(x_{m}, y_{m}\right) \in \partial \Omega_{12}$ are

$$
\begin{aligned}
& G_{\partial \Omega_{12}}^{(0,1)}\left(\mathbf{x}_{m}, t_{m-}\right)<0, \\
& G_{\partial \Omega_{12}}^{(0,2)}\left(\mathbf{x}_{m}, t_{m \pm}\right)=0, \\
& G_{\partial \Omega_{12}}^{(1,2)}\left(\mathbf{x}_{m}, t_{m \pm}\right)<0,
\end{aligned}
$$


From (89) and (90), one obtains

$$
\begin{aligned}
b^{2} x_{m} y_{m}+a^{2} y_{m} F^{(1)}\left(\mathbf{x}_{m}, t_{m-}\right) & <0, \\
b^{2} x_{m} y_{m}+a^{2} y_{m} F^{(2)}\left(\mathbf{x}_{m}, t_{m \pm}\right) & =0, \\
b^{2} x_{m} F^{(2)}\left(\mathbf{x}_{m}, t_{m \pm}\right)+a^{2} y_{m} D F^{(2)}\left(\mathbf{x}_{m}, t_{m \pm}\right) & <0, \\
\text { for } \Omega_{1} & \longrightarrow \partial \Omega_{12} .
\end{aligned}
$$

Thus (80) in (i) holds, and (81) in (i) and (84) and (85) in (iii) can be proved similarly.

According to (27) and (28), the 0th-order $G$-functions and the 1st-order $G$-functions on the boundary $\partial \Omega_{23}$ in domain $\Omega_{2}$ and domain $\Omega_{3}$ are

$$
\begin{aligned}
& G_{\partial \Omega_{23}}^{(0,2)}\left(\mathbf{x}_{m}, t_{m \pm}\right)=\mathbf{n}_{\partial \Omega_{23}}^{\mathrm{T}} \cdot \mathbf{F}^{(2)}\left(\mathbf{x}_{m}, t_{m \pm}\right), \\
& G_{\partial \Omega_{23}}^{(1,2)}\left(\mathbf{x}_{m}, t_{m \pm}\right)=\mathbf{n}_{\partial \Omega_{23}}^{\mathrm{T}} \cdot D \mathbf{F}^{(2)}\left(\mathbf{x}_{m}, t_{m \pm}\right), \\
& \text { in } \Omega_{2} \text { for } \partial \Omega_{23}, \\
& G_{\partial \Omega_{23}}^{(0,3)}\left(\mathbf{x}_{m}, t_{m \pm}\right)=\mathbf{n}_{\partial \Omega_{23}}^{\mathrm{T}} \cdot \mathbf{F}^{(3)}\left(\mathbf{x}_{m}, t_{m \pm}\right), \\
& G_{\partial \Omega_{23}}^{(1,3)}\left(\mathbf{x}_{m}, t_{m \pm}\right)=\mathbf{n}_{\partial \Omega_{23}}^{\mathrm{T}} \cdot D \mathbf{F}^{(3)}\left(\mathbf{x}_{m}, t_{m \pm}\right), \\
& \text { in } \Omega_{3} \text { for } \partial \Omega_{23} .
\end{aligned}
$$

From (13) and (34), (92) can be computed as

$$
\begin{aligned}
G_{\partial \Omega_{23}}^{(0,2)}\left(\mathbf{x}_{m}, t_{m \pm}\right) & =F^{(2)}\left(\mathbf{x}_{m}, t_{m \pm}\right), \\
G_{\partial \Omega_{23}}^{(1,2)}\left(\mathbf{x}_{m}, t_{m \pm}\right) & =D F^{(2)}\left(\mathbf{x}_{m}, t_{m \pm}\right), \\
\quad \text { in } \Omega_{2} \text { for } \partial \Omega_{23}, & \\
G_{\partial \Omega_{23}}^{(0,3)}\left(\mathbf{x}_{m}, t_{m \pm}\right) & =F^{(3)}\left(\mathbf{x}_{m}, t_{m \pm}\right), \\
G_{\partial \Omega_{23}}^{(1,3)}\left(\mathbf{x}_{m}, t_{m \pm}\right) & =D F^{(3)}\left(\mathbf{x}_{m}, t_{m \pm}\right),
\end{aligned}
$$

in $\Omega_{3}$ for $\partial \Omega_{23}$.

By Lemma 6, the appearance conditions for the stick motion of the mass $M$ at $\mathbf{x}_{m} \equiv\left(x_{m}, y_{m}\right) \in \partial \Omega_{23}$ are

$$
\begin{aligned}
& G_{\partial \Omega_{23}}^{(0,2)}\left(\mathbf{x}_{m}, t_{m-}\right)<0, \\
& G_{\partial \Omega_{23}}^{(0,3)}\left(\mathbf{x}_{m}, t_{m \pm}\right)=0, \\
& G_{\partial \Omega_{23}}^{(1,3)}\left(\mathbf{x}_{m}, t_{m \pm}\right)<0,
\end{aligned}
$$

$$
\text { for } \Omega_{2} \longrightarrow \partial \Omega_{23} \text {. }
$$

From (93) and (94), one obtains

$$
\begin{aligned}
F^{(2)}\left(\mathbf{x}_{m}, t_{m-}\right) & <0, \\
F^{(3)}\left(\mathbf{x}_{m}, t_{m \pm}\right) & =0, \\
D F^{(3)}\left(\mathbf{x}_{m}, t_{m \pm}\right) & <0,
\end{aligned}
$$

$$
\text { for } \Omega_{2} \longrightarrow \partial \Omega_{23} \text {. }
$$

Thus (82) in (ii) holds, and (83) in (ii) and (86) and (87) in (iv) can be proved similarly.

Theorem 12. For the friction-induced oscillator with an elliptic control law and the friction force acting on the mass $M$ discussed in Section 2, there are the following results.

(i) The vanishing conditions for the sliding motion of the mass $M$ at $\mathbf{x}_{m} \equiv\left(x_{m}, y_{m}\right) \in \partial \Omega_{12}$ are

$$
\begin{array}{r}
b^{2} x_{m} y_{m}+a^{2} y_{m} F^{(1)}\left(\mathbf{x}_{m}, t_{m \mp}\right)=0, \\
b^{2} x_{m} F^{(1)}\left(\mathbf{x}_{m}, t_{m \mp}\right)+a^{2} y_{m} D F^{(1)}\left(\mathbf{x}_{m}, t_{m \mp}\right)>0, \\
b^{2} x_{m} y_{m}+a^{2} y_{m} F^{(2)}\left(\mathbf{x}_{m}, t_{m-}\right)>0, \\
\text { for } \partial \Omega_{12} \longrightarrow \Omega_{1},
\end{array}
$$

or

$$
\begin{array}{r}
b^{2} x_{m} y_{m}+a^{2} y_{m} F^{(1)}\left(\mathbf{x}_{m}, t_{m-}\right)<0, \\
b^{2} x_{m} y_{m}+a^{2} y_{m} F^{(2)}\left(\mathbf{x}_{m}, t_{m \mp}\right)=0, \\
b^{2} x_{m} F^{(2)}\left(\mathbf{x}_{m}, t_{m \mp}\right)+a^{2} y_{m} D F^{(2)}\left(\mathbf{x}_{m}, t_{m \mp}\right)<0, \\
\text { for } \partial \Omega_{12} \longrightarrow \Omega_{2} .
\end{array}
$$

(ii) The vanishing conditions for the stick motion of the mass $M$ at $\mathbf{x}_{m} \equiv\left(x_{m}, y_{m}\right) \in \partial \Omega_{23}$ are

$$
\begin{aligned}
F^{(2)}\left(\mathbf{x}_{m}, t_{m \mp}\right) & =0, \\
D F^{(2)}\left(\mathbf{x}_{m}, t_{m \mp}\right) & >0, \\
F^{(3)}\left(\mathbf{x}_{m}, t_{m-}\right)>0, & \text { for } \partial \Omega_{23} \longrightarrow \Omega_{2},
\end{aligned}
$$

or

$$
\begin{aligned}
F^{(2)}\left(\mathbf{x}_{m}, t_{m-}\right) & <0, \\
F^{(3)}\left(\mathbf{x}_{m}, t_{m \mp}\right) & =0, \\
D F^{(3)}\left(\mathbf{x}_{m}, t_{m \mp}\right) & <0,
\end{aligned}
$$

(iii) The vanishing conditions for the sliding motion of the mass $M$ at $\mathbf{x}_{m} \equiv\left(x_{m}, y_{m}\right) \in \partial \Omega_{34}$ are

$$
\begin{aligned}
b^{2} x_{m} y_{m}+a^{2} y_{m} F^{(3)}\left(\mathbf{x}_{m}, t_{m \mp}\right) & =0, \\
b^{2} x_{m} F^{(3)}\left(\mathbf{x}_{m}, t_{m \mp}\right)+a^{2} y_{m} D F^{(3)}\left(\mathbf{x}_{m}, t_{m \mp}\right) & <0, \\
b^{2} x_{m} y_{m}+a^{2} y_{m} F^{(4)}\left(\mathbf{x}_{m}, t_{m-}\right) & <0, \\
\text { for } \partial \Omega_{34} & \longrightarrow \Omega_{3},
\end{aligned}
$$


or

$$
\begin{array}{r}
b^{2} x_{m} y_{m}+a^{2} y_{m} F^{(3)}\left(\mathbf{x}_{m}, t_{m-}\right)>0, \\
b^{2} x_{m} y_{m}+a^{2} y_{m} F^{(4)}\left(\mathbf{x}_{m}, t_{m \mp}\right)=0, \\
b^{2} x_{m} F^{(4)}\left(\mathbf{x}_{m}, t_{m \mp}\right)+a^{2} y_{m} D F^{(4)}\left(\mathbf{x}_{m}, t_{m \mp}\right)>0, \\
\text { for } \partial \Omega_{34} \longrightarrow \Omega_{4} .
\end{array}
$$

(iv) The vanishing conditions for the stick motion of the mass $M$ at $\mathbf{x}_{m} \equiv\left(x_{m}, y_{m}\right) \in \partial \Omega_{14}$ are

$$
\begin{aligned}
F^{(1)}\left(\mathbf{x}_{m}, t_{m \mp}\right) & =0, \\
D F^{(1)}\left(\mathbf{x}_{m}, t_{m \mp}\right)>0, & \\
F^{(4)}\left(\mathbf{x}_{m}, t_{m-}\right)>0, & \text { for } \partial \Omega_{14} \longrightarrow \Omega_{1},
\end{aligned}
$$

or

$$
\begin{aligned}
F^{(1)}\left(\mathbf{x}_{m}, t_{m-}\right) & <0, \\
F^{(4)}\left(\mathbf{x}_{m}, t_{m \mp}\right) & =0, \\
D F^{(4)}\left(\mathbf{x}_{m}, t_{m \mp}\right) & <0,
\end{aligned}
$$

$$
\text { for } \partial \Omega_{14} \longrightarrow \Omega_{4} \text {. }
$$

Proof. The mass $M$ and the conveyor belt move together for a long time, at time $t_{m}$, the nonfriction force acting on the mass $M$ is to overcome static friction, and the mass $M$ begins to move with nonzero relative velocity. From the flow switchability theory on the discontinuous dynamical systems, the sliding or stick motion vanishes, which means that the flow on the boundary $\partial \Omega_{\alpha \beta}(\alpha \beta \in\{12,21,34,43\})$ or $\partial \Omega_{i j}(i j \in\{23,32,14,41\})$ will go into domain $\Omega_{\lambda}(\lambda \in$ $\{\alpha, \beta\})$ or $\Omega_{\gamma}(\gamma \in\{i, j\})$, and time $t_{m}$ is the switching time from nonpassable flow of the first kind to semipassable flow. Thus it can be predicted by Lemma 7. So the 0th-order Gfunctions and the 1st-order $G$-functions on such boundaries are needed.

According to (27) and (28), the 0th-order $G$-functions and the 1st-order $G$-functions on the boundary $\partial \Omega_{12}$ in domain $\Omega_{1}$ and domain $\Omega_{2}$ are

$$
\begin{aligned}
& G_{\partial \Omega_{12}}^{(0,1)}\left(\mathbf{x}_{m}, t_{m \pm}\right)=\mathbf{n}_{\partial \Omega_{12}}^{\mathrm{T}} \cdot \mathbf{F}^{(1)}\left(\mathbf{x}_{m}, t_{m \pm}\right), \\
& G_{\partial \Omega_{12}}^{(1,1)}\left(\mathbf{x}_{m}, t_{m \pm}\right)=\mathbf{n}_{\partial \Omega_{12}}^{\mathrm{T}} \cdot D \mathbf{F}^{(1)}\left(\mathbf{x}_{m}, t_{m \pm}\right), \\
& \quad \text { in } \Omega_{1} \text { for } \partial \Omega_{12}, \\
& G_{\partial \Omega_{12}}^{(0,2)}\left(\mathbf{x}_{m}, t_{m \pm}\right)=\mathbf{n}_{\partial \Omega_{12}}^{\mathrm{T}} \cdot \mathbf{F}^{(2)}\left(\mathbf{x}_{m}, t_{m \pm}\right), \\
& G_{\partial \Omega_{12}}^{(1,2)}\left(\mathbf{x}_{m}, t_{m \pm}\right)=\mathbf{n}_{\partial \Omega_{12}}^{\mathrm{T}} \cdot D \mathbf{F}^{(2)}\left(\mathbf{x}_{m}, t_{m \pm}\right),
\end{aligned}
$$

From (13) and (35), (104) can be computed as

$$
\begin{aligned}
& G_{\partial \Omega_{12}}^{(0,1)}\left(\mathbf{x}_{m}, t_{m \pm}\right)= b^{2} x_{m} y_{m}+a^{2} y_{m} F^{(1)}\left(\mathbf{x}_{m}, t_{m \pm}\right), \\
& G_{\partial \Omega_{12}}^{(1,1)}\left(\mathbf{x}_{m}, t_{m \pm}\right)= b^{2} x_{m} F^{(1)}\left(\mathbf{x}_{m}, t_{m \pm}\right) \\
&+a^{2} y_{m} D F^{(1)}\left(\mathbf{x}_{m}, t_{m \pm}\right), \\
& \text { in } \Omega_{1} \text { for } \partial \Omega_{12}, \\
& G_{\partial \Omega_{12}}^{(0,2)}\left(\mathbf{x}_{m}, t_{m \pm}\right)= b^{2} x_{m} y_{m}+a^{2} y_{m} F^{(2)}\left(\mathbf{x}_{m}, t_{m \pm}\right), \\
& G_{\partial \Omega_{12}}^{(1,2)}\left(\mathbf{x}_{m}, t_{m \pm}\right)= b^{2} x_{m} F^{(2)}\left(\mathbf{x}_{m}, t_{m \pm}\right) \\
&+a^{2} y_{m} D F^{(2)}\left(\mathbf{x}_{m}, t_{m \pm}\right), \\
& \text { in } \Omega_{2} \text { for } \partial \Omega_{12} .
\end{aligned}
$$

By Lemma 7, the vanishing conditions for the sliding motion of the mass $M$ at $\mathbf{x}_{m} \equiv\left(x_{m}, y_{m}\right) \in \partial \Omega_{12}$ are

$$
\begin{aligned}
& G_{\partial \Omega_{12}}^{(0,1)}\left(\mathbf{x}_{m}, t_{m \mp}\right)=0, \\
& G_{\partial \Omega_{12}}^{(1,1)}\left(\mathbf{x}_{m}, t_{m \mp}\right)>0, \\
& G_{\partial \Omega_{12}}^{(0,2)}\left(\mathbf{x}_{m}, t_{m-}\right)>0,
\end{aligned}
$$

for $\partial \Omega_{12} \longrightarrow \Omega_{1}$.

From (105) and (106), one obtains

$$
\begin{aligned}
b^{2} x_{m} y_{m}+a^{2} y_{m} F^{(1)}\left(\mathbf{x}_{m}, t_{m \mp}\right) & =0, \\
b^{2} x_{m} F^{(1)}\left(\mathbf{x}_{m}, t_{m \mp}\right)+a^{2} y_{m} D F^{(1)}\left(\mathbf{x}_{m}, t_{m \mp}\right) & >0, \\
b^{2} x_{m} y_{m}+a^{2} y_{m} F^{(2)}\left(\mathbf{x}_{m}, t_{m-}\right) & >0, \\
\text { for } \partial \Omega_{12} & \longrightarrow \Omega_{1} .
\end{aligned}
$$

Thus (96) in (i) holds, and (97) in (i) and (100) and (101) in (iii) can be proved similarly.

By (27) and (28), the 0th-order G-functions and the 1storder $G$-functions on the boundary $\partial \Omega_{23}$ in domain $\Omega_{2}$ and domain $\Omega_{3}$ are

$$
\begin{aligned}
& G_{\partial \Omega_{23}}^{(0,2)}\left(\mathbf{x}_{m}, t_{m \pm}\right)=\mathbf{n}_{\partial \Omega_{23}}^{\mathrm{T}} \cdot \mathbf{F}^{(2)}\left(\mathbf{x}_{m}, t_{m \pm}\right), \\
& G_{\partial \Omega_{23}}^{(1,2)}\left(\mathbf{x}_{m}, t_{m \pm}\right)=\mathbf{n}_{\partial \Omega_{23}}^{\mathrm{T}} \cdot D \mathbf{F}^{(2)}\left(\mathbf{x}_{m}, t_{m \pm}\right),
\end{aligned}
$$
in $\Omega_{2}$ for $\partial \Omega_{23}$

$$
\begin{aligned}
& G_{\partial \Omega_{23}}^{(0,3)}\left(\mathbf{x}_{m}, t_{m \pm}\right)=\mathbf{n}_{\partial \Omega_{23}}^{\mathrm{T}} \cdot \mathbf{F}^{(3)}\left(\mathbf{x}_{m}, t_{m \pm}\right), \\
& G_{\partial \Omega_{23}}^{(1,3)}\left(\mathbf{x}_{m}, t_{m \pm}\right)=\mathbf{n}_{\partial \Omega_{23}}^{\mathrm{T}} \cdot D \mathbf{F}^{(3)}\left(\mathbf{x}_{m}, t_{m \pm}\right),
\end{aligned}
$$


From (13) and (34), (108) can be computed as

$$
\begin{aligned}
& G_{\partial \Omega_{23}}^{(0,2)}\left(\mathbf{x}_{m}, t_{m \pm}\right)=F^{(2)}\left(\mathbf{x}_{m}, t_{m \pm}\right), \\
& G_{\partial \Omega_{23}}^{(1,2)}\left(\mathbf{x}_{m}, t_{m \pm}\right)=D F^{(2)}\left(\mathbf{x}_{m}, t_{m \pm}\right), \\
& \text { in } \Omega_{2} \text { for } \partial \Omega_{23}, \\
& G_{\partial \Omega_{23}}^{(0,3)}\left(\mathbf{x}_{m}, t_{m \pm}\right)=F^{(3)}\left(\mathbf{x}_{m}, t_{m \pm}\right), \\
& G_{\partial \Omega_{23}}^{(1,3)}\left(\mathbf{x}_{m}, t_{m \pm}\right)=D F^{(3)}\left(\mathbf{x}_{m}, t_{m \pm}\right),
\end{aligned}
$$

in $\Omega_{3}$ for $\partial \Omega_{23}$.

By Lemma 7, the vanishing conditions for the stick motion of the mass $M$ at $\mathbf{x}_{m} \equiv\left(x_{m}, y_{m}\right) \in \partial \Omega_{23}$ are

$$
\begin{aligned}
& G_{\partial \Omega_{23}}^{(0,2)}\left(\mathbf{x}_{m}, t_{m \mp}\right)=0, \\
& G_{\partial \Omega_{23}}^{(1,2)}\left(\mathbf{x}_{m}, t_{m \mp}\right)>0, \\
& G_{\partial \Omega_{23}}^{(0,3)}\left(\mathbf{x}_{m}, t_{m-}\right)>0,
\end{aligned}
$$

$$
\text { for } \partial \Omega_{23} \longrightarrow \Omega_{2} \text {. }
$$

From (109) and (110), one obtains

$$
\begin{aligned}
F^{(2)}\left(\mathbf{x}_{m}, t_{m \mp}\right) & =0, \\
D F^{(2)}\left(\mathbf{x}_{m}, t_{m \mp}\right) & >0, \\
F^{(3)}\left(\mathbf{x}_{m}, t_{m-}\right) & >0,
\end{aligned}
$$

$$
\text { for } \partial \Omega_{23} \longrightarrow \Omega_{2} \text {. }
$$

Thus (98) in (ii) holds, and (99) in (ii) and (102) and (103) in (iv) can be proved similarly.

\section{Mapping Structures and Periodic Motions}

In order to describe the periodic motions with or without stick or sliding in the friction-induced oscillator with an elliptic control law and the friction acting on the mass $M$ discussed in Section 2, the mapping structures are introduced based on the discontinuous boundaries. The switching sets will be defined firstly. From the switching sets, the basic mappings will be defined for this discontinuous dynamical system.

Using the discontinuous boundaries $\partial \Omega_{\alpha_{1} \alpha_{2}}\left(\alpha_{1} \alpha_{2} \in\right.$ $\{12,21,23,32,34,43,14,41\})$ in (12), the switching sets of mass $M$ are defined as

$$
\begin{aligned}
\Sigma_{12} & =\Sigma_{21} \\
& =\left\{\left(x_{m}, \dot{x}_{m}, t_{m}\right) \mid \frac{x_{m}^{2}}{a^{2}}+\frac{\dot{x}_{m}^{2}}{b^{2}}-1=0, \dot{x}_{m}>V\right\}, \\
\Sigma_{23} & =\Sigma_{32} \\
& =\left\{\left(x_{m}, \dot{x}_{m}, t_{m}\right) \mid \frac{x_{m}^{2}}{a^{2}}+\frac{\dot{x}_{m}^{2}}{b^{2}}-1<0, \dot{x}_{m}=V\right\},
\end{aligned}
$$

$$
\begin{aligned}
\Sigma_{34} & =\Sigma_{43} \\
& =\left\{\left(x_{m}, \dot{x}_{m}, t_{m}\right) \mid \frac{x_{m}^{2}}{a^{2}}+\frac{\dot{x}_{m}^{2}}{b^{2}}-1=0, \dot{x}_{m}<V\right\}, \\
\Sigma_{14} & =\Sigma_{41} \\
& =\left\{\left(x_{m}, \dot{x}_{m}, t_{m}\right) \mid \frac{x_{m}^{2}}{a^{2}}+\frac{\dot{x}_{m}^{2}}{b^{2}}-1>0, \dot{x}_{m}=V\right\}, \\
\Sigma_{12}^{+} & \\
& =\left\{\left(x_{m}, \dot{x}_{m}, t_{m}\right) \mid \frac{x_{m}^{2}}{a^{2}}+\frac{\dot{x}_{m}^{2}}{b^{2}}-1=0^{+}, \dot{x}_{m}>V\right\},
\end{aligned}
$$

$\Sigma_{21}^{-}$

$$
=\left\{\left(x_{m}, \dot{x}_{m}, t_{m}\right) \mid \frac{x_{m}^{2}}{a^{2}}+\frac{\dot{x}_{m}^{2}}{b^{2}}-1=0^{-}, \dot{x}_{m}>V\right\},
$$

$\Sigma_{23}^{+}$

$$
=\left\{\left(x_{m}, \dot{x}_{m}, t_{m}\right) \mid \frac{x_{m}^{2}}{a^{2}}+\frac{\dot{x}_{m}^{2}}{b^{2}}-1<0, \dot{x}_{m}=V^{+}\right\},
$$

$\Sigma_{32}^{-}$

$$
=\left\{\left(x_{m}, \dot{x}_{m}, t_{m}\right) \mid \frac{x_{m}^{2}}{a^{2}}+\frac{\dot{x}_{m}^{2}}{b^{2}}-1<0, \dot{x}_{m}=V^{-}\right\},
$$

$\Sigma_{34}^{-}$

$$
=\left\{\left(x_{m}, \dot{x}_{m}, t_{m}\right) \mid \frac{x_{m}^{2}}{a^{2}}+\frac{\dot{x}_{m}^{2}}{b^{2}}-1=0^{-}, \dot{x}_{m}<V\right\},
$$

$\Sigma_{43}^{+}$

$$
=\left\{\left(x_{m}, \dot{x}_{m}, t_{m}\right) \mid \frac{x_{m}^{2}}{a^{2}}+\frac{\dot{x}_{m}^{2}}{b^{2}}-1=0^{+}, \dot{x}_{m}<V\right\},
$$

$\Sigma_{14}^{+}$

$$
=\left\{\left(x_{m}, \dot{x}_{m}, t_{m}\right) \mid \frac{x_{m}^{2}}{a^{2}}+\frac{\dot{x}_{m}^{2}}{b^{2}}-1>0, \dot{x}_{m}=V^{+}\right\},
$$

$\Sigma_{41}^{-}$

$$
=\left\{\left(x_{m}, \dot{x}_{m}, t_{m}\right) \mid \frac{x_{m}^{2}}{a^{2}}+\frac{\dot{x}_{m}^{2}}{b^{2}}-1>0, \dot{x}_{m}=V^{-}\right\},
$$

where $x_{m}$ and $\dot{x}_{m}(m \in \mathbf{N})$ are switching displacement and velocity on the corresponding discontinuous boundaries for the mass $M$ at time $t_{m}$, and $V^{ \pm}=\lim _{\delta \rightarrow 0^{+}}(V \pm \delta)$ and $0^{ \pm}=$ $\lim _{\delta \rightarrow 0^{+}}(0 \pm \delta)$.

From the above switching sets, the basic mappings can be defined as

$$
P_{a}: \Sigma_{12}^{+} \longrightarrow \Sigma_{14}^{+} \text {, }
$$




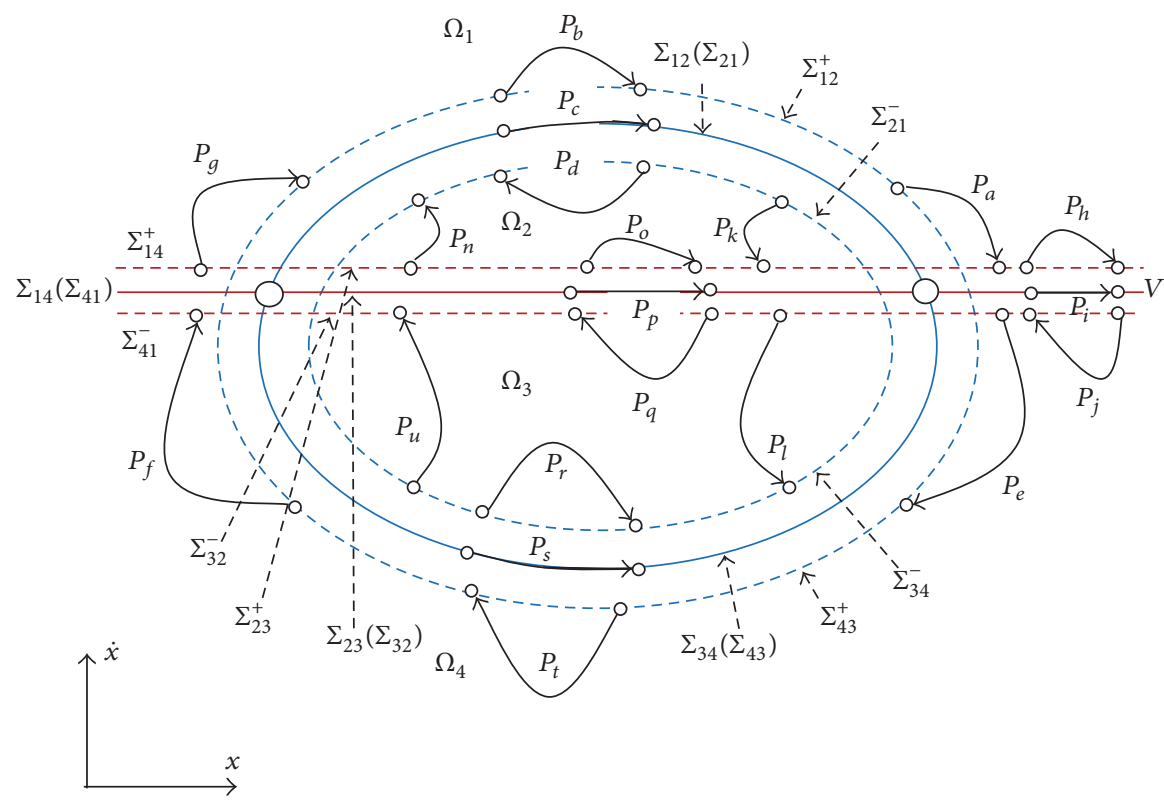

FIGURE 4: The switching sets and basic mappings in phase plane.

$$
\begin{aligned}
& P_{b}: \Sigma_{12}^{+} \longrightarrow \Sigma_{12}^{+}, \\
& P_{c}: \Sigma_{12} \longrightarrow \Sigma_{12}, \\
& P_{d}: \Sigma_{21}^{-} \longrightarrow \Sigma_{21}^{-}, \\
& P_{e}: \Sigma_{41}^{-} \longrightarrow \Sigma_{43}^{+}, \\
& P_{f}: \Sigma_{43}^{+} \longrightarrow \Sigma_{41}^{-}, \\
& P_{g}: \Sigma_{14}^{+} \longrightarrow \Sigma_{12}^{+}, \\
& P_{h}: \Sigma_{14}^{+} \longrightarrow \Sigma_{14}^{+}, \\
& P_{i}: \Sigma_{14} \longrightarrow \Sigma_{14}, \\
& P_{j}: \Sigma_{41}^{-} \longrightarrow \Sigma_{41}^{-}, \\
& P_{k}: \Sigma_{21}^{-} \longrightarrow \Sigma_{23}^{+}, \\
& P_{l}: \Sigma_{32}^{-} \longrightarrow \Sigma_{34}^{-}, \\
& P_{u}: \Sigma_{34}^{-} \longrightarrow \Sigma_{32}^{-}, \\
& P_{n}: \Sigma_{23}^{+} \longrightarrow \Sigma_{21}^{-}, \\
& P_{o}: \Sigma_{23}^{+} \longrightarrow \Sigma_{23}^{+}, \\
& P_{p}: \Sigma_{23} \longrightarrow \Sigma_{23}, \\
& P_{q}: \Sigma_{32}^{-} \longrightarrow \Sigma_{32}^{-}, \\
& P_{r}: \Sigma_{34}^{-} \longrightarrow \Sigma_{34}^{-}, \\
& P_{s}: \Sigma_{34} \longrightarrow \Sigma_{34}, \\
& P_{t}: \Sigma_{43}^{+} \longrightarrow \Sigma_{43}^{+} .
\end{aligned}
$$

From the above definitions, the switching sets and basic mappings are sketched in Figure 4.

The corresponding mapping relationships are

$$
\begin{aligned}
& P_{\theta}:\left(x_{m}, \dot{x}_{m}, t_{m}\right) \longrightarrow\left(x_{m+1}, \dot{x}_{m+1}, t_{m+1}\right) \\
& \text { for } \theta \in\{a, b, c, d, e, f, g, h, i, j, k, l, n, o, p, q, r, s, t, u\} .
\end{aligned}
$$

In the above basic mappings, $P_{\theta}(\theta \in\{b, c, d, h, i, j, o, p, q, r$, $s, t\})$ are the local mappings that map from one switching set to itself. And $P_{\theta}(\theta \in\{a, e, f, g, k, l, u, n\})$ are the global mappings that map from one switching set to another.

Combined with the elliptic control law in (1) and its solution, the governing equations for the sliding mapping $P_{c}$ are

$$
\begin{aligned}
& x_{m+1}=a \sin \left(\frac{b}{a}\left(t_{m+1}-t_{m}\right)+\arcsin \frac{x_{m}}{a}\right), \\
& y_{m+1}=b \cos \left(\frac{b}{a}\left(t_{m+1}-t_{m}\right)+\arcsin \frac{x_{m}}{a}\right), \\
& G_{\partial \Omega_{12}}^{(0, i)}\left(\mathbf{x}_{m+1}, t_{m+1}\right)=0, \\
& (-1)^{i} G_{\partial \Omega_{12}}^{(1, i)}\left(\mathbf{x}_{m+1}, t_{m+1}\right)<0, \\
& G_{\partial \Omega_{12}}^{(0,1)}(\mathbf{x}, t)<0, \\
& G_{\partial \Omega_{12}}^{(0,2)}(\mathbf{x}, t)>0,
\end{aligned}
$$

for $\mathbf{x}_{m+1}=\left(x_{m+1}, y_{m+1}\right), \mathbf{x}_{m}=\left(x_{m}, y_{m}\right), i=1$ or $2, \mathbf{x}=(x, y)$, $t \in\left(t_{m}, t_{m+1}\right), x \in\left(x_{m}, x_{m+1}\right)$, and $y \in\left(y_{m}, y_{m+1}\right)$ on $\partial \Omega_{12}$, and the governing equations for the sliding mapping $P_{s}$ can be defined similarly. 
In general, the governing equations for basic mappings,

$P_{\theta}$

$$
(\theta \in\{a, b, c, d, e, f, g, h, i, j, k, l, n, o, p, q, r, s, t, u\}),
$$

can be expressed by

$$
\mathbf{f}^{(\theta)}\left(\mathbf{Y}_{m}, \mathbf{Y}_{m+1}\right)=0
$$

with

$$
\begin{aligned}
& \mathbf{f}^{(\theta)}=\left(f_{1}^{(\theta)}, f_{2}^{(\theta)}\right)^{\mathrm{T}}, \\
& \mathbf{Y}_{m}=\left(x_{m}, \dot{x}_{m}, t_{m}\right)^{\mathrm{T}}, \\
& \mathbf{Y}_{m+1}=\left(x_{m+1}, \dot{x}_{m+1}, t_{m+1}\right)^{\mathrm{T}}, \\
& f_{1}^{(\theta)}\left(x_{m}, y_{m}, t_{m}, x_{m+1}, y_{m+1}, t_{m+1}\right)=0, \\
& f_{2}^{(\theta)}\left(x_{m}, y_{m}, t_{m}, x_{m+1}, y_{m+1}, t_{m+1}\right)=0,
\end{aligned}
$$

$$
\begin{aligned}
& \frac{x_{m}^{2}}{a^{2}}+\frac{\dot{x}_{m}^{2}}{b^{2}}=1 \quad \text { or } \dot{x}_{m}=V, \\
& \frac{x_{m+1}^{2}}{a^{2}}+\frac{\dot{x}_{m+1}^{2}}{b^{2}}=1 \quad \text { or } \dot{x}_{m+1}=V .
\end{aligned}
$$

The notation for mapping structure is introduced as

$$
P_{n_{1} n_{2} \cdots n_{w}} \equiv P_{n_{1}} \circ P_{n_{2}} \circ \cdots \circ P_{n_{w}},
$$

where $n_{i} \in\{a, b, c, d, e, f, g, h, i, j, k, l, n, o, p, q, r, s, t, u\}, i \in$ $\{1,2,3, \ldots, w\}$. The motion of the $N$-terms repeating of mapping $P_{n_{1} n_{2} \cdots n_{w}}$ is defined as

$$
\begin{aligned}
& P_{n_{1} n_{2} \cdots n_{w}}^{(N)} \\
& \equiv \underbrace{\left(P_{n_{1}} \circ P_{n_{2}} \circ \cdots \circ P_{n_{w}}\right) \circ \cdots \circ\left(P_{n_{1}} \circ P_{n_{2}} \circ \cdots \circ P_{n_{w}}\right)}_{N \text {-terms }} .
\end{aligned}
$$

Now, consider a periodic orbit formed by generic mappings in phase plane, and a generalized mapping structure is assumed as

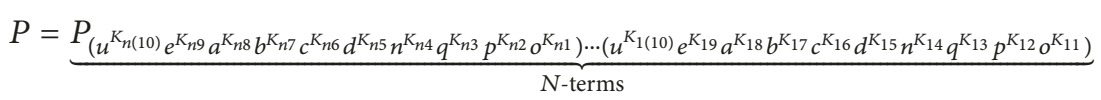

for $N \in Z^{+}, K_{k \tau} \in\{0,1\}, \tau \in\{1,2,3, \ldots, 10\}$, and $k \in$ $\{1,2,3, \ldots, n\}$.

In what follows, four simple periodic orbits with or without sliding or stick motions will be presented to explain how to form periodic motions in the discontinuous dynamic system.

Suppose $N=1, K_{17}=K_{16}=K_{15}=K_{13}=K_{12}=$ $K_{11}=0$, and $K_{1(10)}=K_{19}=K_{18}=K_{14}=1$, and then there is a mapping structure for a passable periodic motion, as shown in Figure 5(a). By using notation in (121), the mapping structure of such a periodic orbit is

$$
P=P_{\text {uean }}=P_{u} \circ P_{e} \circ P_{a} \circ P_{n} .
$$

The mapping relations are

$$
\begin{array}{r}
P_{n}:\left(x_{m}, \dot{x}_{m}, t_{m}\right) \longrightarrow\left(x_{m+1}, \dot{x}_{m+1}, t_{m+1}\right), \\
P_{a}:\left(x_{m+1}, \dot{x}_{m+1}, t_{m+1}\right) \longrightarrow\left(x_{m+2}, \dot{x}_{m+2}, t_{m+2}\right), \\
P_{e}:\left(x_{m+2}, \dot{x}_{m+2}, t_{m+2}\right) \longrightarrow\left(x_{m+3}, \dot{x}_{m+3}, t_{m+3}\right), \\
P_{u}:\left(x_{m+3}, \dot{x}_{m+3}, t_{m+3}\right) \longrightarrow\left(x_{m+4}, \dot{x}_{m+4}, t_{m+4}\right) .
\end{array}
$$

During $N$-period time of excitation, the periodicity of mapping $P$ requires

$$
\begin{aligned}
& x_{m+4}=x_{m}, \\
& \dot{x}_{m+4}=\dot{x}_{m}, \\
& t_{m+4}=t_{m}+\frac{2 N \pi}{\Omega} .
\end{aligned}
$$

Therefore, based on the governing equations in (117), the nonlinear algebraic equations for such a mapping structure $P_{\text {uean }}$ can be obtained, that is, (124) plus

$$
\begin{aligned}
\frac{x_{m+1}^{2}}{a^{2}}+\frac{\dot{x}_{m+1}^{2}}{b^{2}} & =1, \\
\frac{x_{m+3}^{2}}{a^{2}}+\frac{\dot{x}_{m+3}^{2}}{b^{2}} & =1, \\
\mathbf{f}^{(n)} & =\left(x_{m}, \dot{x}_{m}, t_{m}, x_{m+1}, \dot{x}_{m+1}, t_{m+1}\right)=0, \\
\mathbf{f}^{(a)} & =\left(x_{m+1}, \dot{x}_{m+1}, t_{m+1}, x_{m+2}, \dot{x}_{m+2}, t_{m+2}\right) \\
& =0, \\
\mathbf{f}^{(e)} & =\left(x_{m+2}, \dot{x}_{m+2}, t_{m+2}, x_{m+3}, \dot{x}_{m+3}, t_{m+3}\right) \\
& =0, \\
\mathbf{f}^{(u)} & =\left(x_{m+3}, \dot{x}_{m+3}, t_{m+3}, x_{m+4}, \dot{x}_{m+4}, t_{m+4}\right) \\
& =0 .
\end{aligned}
$$

Consider a mapping structure for a sliding periodic orbit with $N=1, K_{1(10)}=K_{19}=K_{18}=K_{14}=K_{13}=K_{12}=K_{11}=$ 0 , and $K_{17}=K_{16}=K_{15}=1$ in (121), as shown in Figure 5(b). Such a periodic orbit can be described as

$$
P=P_{b c d}=P_{b} \circ P_{c} \circ P_{d},
$$




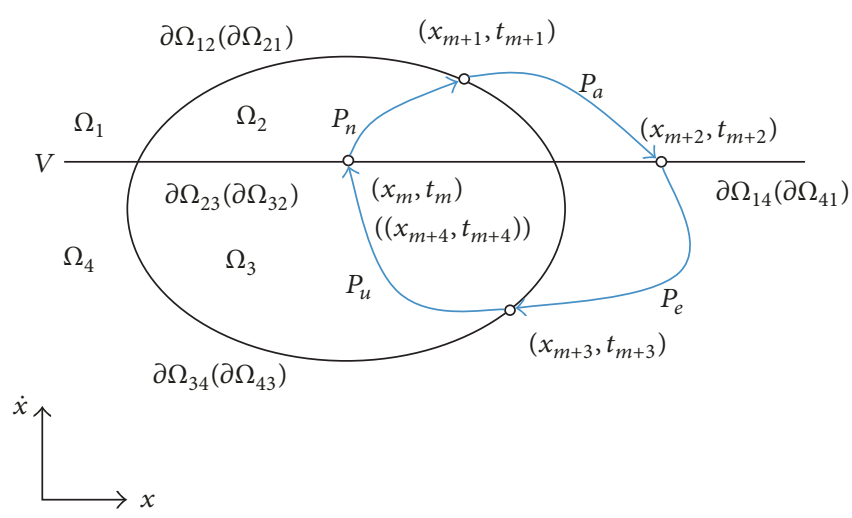

(a)

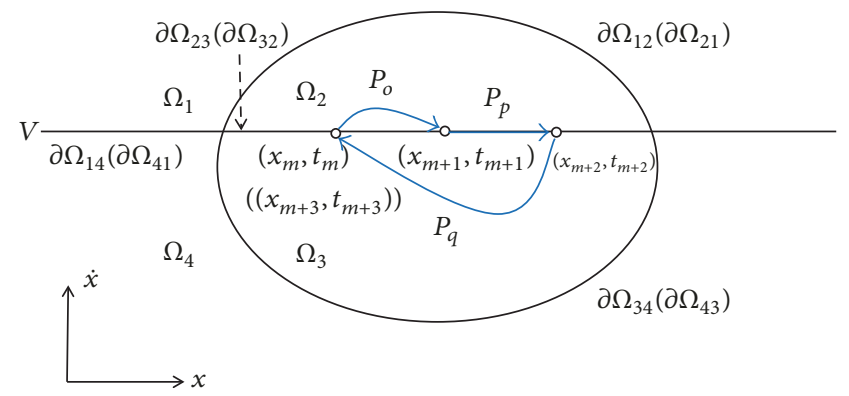

(c)

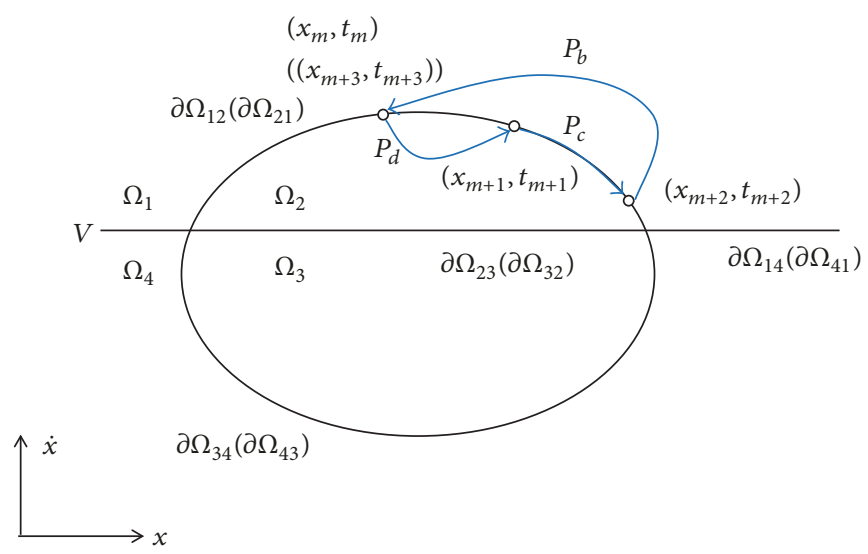

(b)

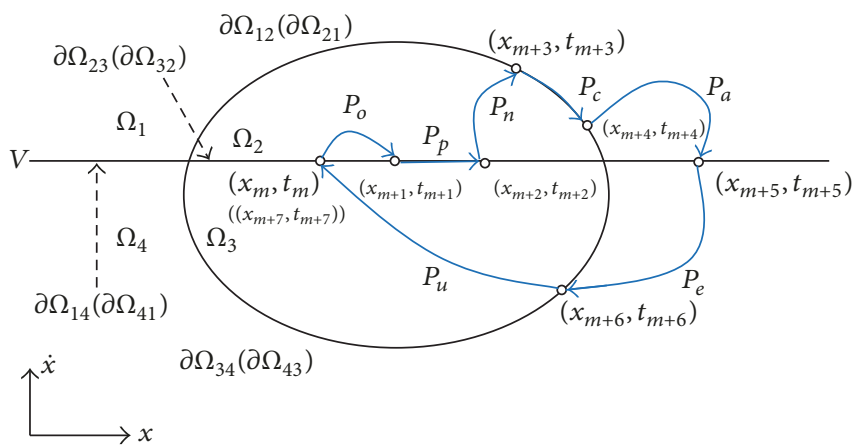

(d)

FIgURE 5: Mapping structures of periodic orbits: (a) $P=P_{\text {uean }}=P_{u} \circ P_{e} \circ P_{a} \circ P_{n}$, (b) $P=P_{b c d}=P_{b} \circ P_{c} \circ P_{d}$, (c) $P=P_{q p o}=P_{q} \circ P_{p} \circ P_{o}$, and (d) $P=P_{\text {ueacnpo }}=P_{u} \circ P_{e} \circ P_{a} \circ P_{c} \circ P_{n} \circ P_{p} \circ P_{o}$.

where

$$
\begin{array}{r}
P_{d}:\left(x_{m}, \dot{x}_{m}, t_{m}\right) \longrightarrow\left(x_{m+1}, \dot{x}_{m+1}, t_{m+1}\right), \\
P_{c}:\left(x_{m+1}, \dot{x}_{m+1}, t_{m+1}\right) \longrightarrow\left(x_{m+2}, \dot{x}_{m+2}, t_{m+2}\right), \\
P_{b}:\left(x_{m+2}, \dot{x}_{m+2}, t_{m+2}\right) \longrightarrow\left(x_{m+3}, \dot{x}_{m+3}, t_{m+3}\right) .
\end{array}
$$

During $N$-period time of excitation, the periodic motion pertaining to such a mapping $P$ requires

$$
\begin{aligned}
& x_{m+3}=x_{m}, \\
& \dot{x}_{m+3}=\dot{x}_{m}, \\
& t_{m+3}=t_{m}+\frac{2 N \pi}{\Omega} .
\end{aligned}
$$

Thus, according to the governing equations in (117), the nonlinear algebraic equations for such a mapping structure $P_{b c d}$ can be obtained, that is, (128) plus

$$
\begin{aligned}
\frac{x_{m}^{2}}{a^{2}}+\frac{\dot{x}_{m}^{2}}{b^{2}} & =1, \\
\frac{x_{m+1}^{2}}{a^{2}}+\frac{\dot{x}_{m+1}^{2}}{b^{2}} & =1, \\
\frac{x_{m+2}^{2}}{a^{2}}+\frac{\dot{x}_{m+2}^{2}}{b^{2}} & =1,
\end{aligned}
$$

$$
\begin{aligned}
\mathbf{f}^{(d)} & =\left(x_{m}, \dot{x}_{m}, t_{m}, x_{m+1}, \dot{x}_{m+1}, t_{m+1}\right)=0, \\
\mathbf{f}^{(c)} & =\left(x_{m+1}, \dot{x}_{m+1}, t_{m+1}, x_{m+2}, \dot{x}_{m+2}, t_{m+2}\right) \\
& =0, \\
\mathbf{f}^{(b)} & =\left(x_{m+2}, \dot{x}_{m+2}, t_{m+2}, x_{m+3}, \dot{x}_{m+3}, t_{m+3}\right) \\
& =0 .
\end{aligned}
$$

Consider a mapping structure for a stick periodic orbit with $N=1, K_{1(10)}=K_{19}=K_{18}=K_{17}=K_{16}=K_{15}=K_{14}=$ 0 , and $K_{13}=K_{12}=K_{11}=1$ in (121), as shown in Figure 5(c). Such a periodic orbit can be expressed as

$$
P=P_{q p o}=P_{q} \circ P_{p} \circ P_{o}
$$

where

$$
\begin{array}{r}
P_{o}:\left(x_{m}, \dot{x}_{m}, t_{m}\right) \longrightarrow\left(x_{m+1}, \dot{x}_{m+1}, t_{m+1}\right), \\
P_{p}:\left(x_{m+1}, \dot{x}_{m+1}, t_{m+1}\right) \longrightarrow\left(x_{m+2}, \dot{x}_{m+2}, t_{m+2}\right), \\
P_{q}:\left(x_{m+2}, \dot{x}_{m+2}, t_{m+2}\right) \longrightarrow\left(x_{m+3}, \dot{x}_{m+3}, t_{m+3}\right) .
\end{array}
$$


During $N$-period time of excitation, the periodic motion pertaining to such a mapping $P$ requires

$$
\begin{aligned}
& x_{m+3}=x_{m}, \\
& \dot{x}_{m+3}=\dot{x}_{m}, \\
& t_{m+3}=t_{m}+\frac{2 N \pi}{\Omega} .
\end{aligned}
$$

Therefore, from the governing equations in (117), one can obtain a set of nonlinear algebraic equations for such a mapping structure $P_{q p o}$, that is, (132) plus

$$
\begin{aligned}
& \mathbf{f}^{(o)}=\left(x_{m}, \dot{x}_{m}, t_{m}, x_{m+1}, \dot{x}_{m+1}, t_{m+1}\right)=0, \\
& \mathbf{f}^{(p)}=\left(x_{m+1}, \dot{x}_{m+1}, t_{m+1}, x_{m+2}, \dot{x}_{m+2}, t_{m+2}\right)=0, \\
& \mathbf{f}^{(q)}=\left(x_{m+2}, \dot{x}_{m+2}, t_{m+2}, x_{m+3}, \dot{x}_{m+3}, t_{m+3}\right)=0 .
\end{aligned}
$$

Consider a mapping structure for stick and sliding periodic orbit with $N=1, K_{1(10)}=K_{19}=K_{18}=K_{16}=K_{14}=$ $K_{12}=K_{11}=1$, and $K_{17}=K_{15}=K_{13}=0$ in (121), as shown in Figure 5(d). Such a periodic orbit can be shown as

$$
P=P_{\text {ueacnpo }}=P_{u} \circ P_{e} \circ P_{a} \circ P_{c} \circ P_{n} \circ P_{p} \circ P_{o},
$$

where

$$
\begin{array}{r}
P_{o}:\left(x_{m}, \dot{x}_{m}, t_{m}\right) \longrightarrow\left(x_{m+1}, \dot{x}_{m+1}, t_{m+1}\right), \\
P_{p}:\left(x_{m+1}, \dot{x}_{m+1}, t_{m+1}\right) \longrightarrow\left(x_{m+2}, \dot{x}_{m+2}, t_{m+2}\right), \\
P_{n}:\left(x_{m+2}, \dot{x}_{m+2}, t_{m+2}\right) \longrightarrow\left(x_{m+3}, \dot{x}_{m+3}, t_{m+3}\right), \\
P_{c}:\left(x_{m+3}, \dot{x}_{m+3}, t_{m+3}\right) \longrightarrow\left(x_{m+4}, \dot{x}_{m+4}, t_{m+4}\right), \\
P_{a}:\left(x_{m+4}, \dot{x}_{m+4}, t_{m+4}\right) \longrightarrow\left(x_{m+5}, \dot{x}_{m+5}, t_{m+5}\right), \\
P_{e}:\left(x_{m+5}, \dot{x}_{m+5}, t_{m+5}\right) \longrightarrow\left(x_{m+6}, \dot{x}_{m+6}, t_{m+6}\right), \\
P_{u}:\left(x_{m+6}, \dot{x}_{m+6}, t_{m+6}\right) \longrightarrow\left(x_{m+7}, \dot{x}_{m+7}, t_{m+7}\right) .
\end{array}
$$

During $N$-period time of excitation, the requirement for the periodic motion of a mapping $P$ is

$$
\begin{aligned}
& x_{m+7}=x_{m}, \\
& \dot{x}_{m+7}=\dot{x}_{m}, \\
& t_{m+7}=t_{m}+\frac{2 N \pi}{\Omega} .
\end{aligned}
$$

So, based on the governing equations in (117), one can show a class of nonlinear algebraic equations for such a mapping structure $P_{\text {ueacnpo }}$, that is, (136) plus

$$
\begin{aligned}
& \frac{x_{m+3}^{2}}{a^{2}}+\frac{\dot{x}_{m+3}^{2}}{b^{2}}=1, \\
& \frac{x_{m+4}^{2}}{a^{2}}+\frac{\dot{x}_{m+4}^{2}}{b^{2}}=1, \\
& \frac{x_{m+6}^{2}}{a^{2}}+\frac{\dot{x}_{m+6}^{2}}{b^{2}}=1,
\end{aligned}
$$

$$
\begin{aligned}
\mathbf{f}^{(o)} & =\left(x_{m}, \dot{x}_{m}, t_{m}, x_{m+1}, \dot{x}_{m+1}, t_{m+1}\right)=0, \\
\mathbf{f}^{(p)} & =\left(x_{m+1}, \dot{x}_{m+1}, t_{m+1}, x_{m+2}, \dot{x}_{m+2}, t_{m+2}\right) \\
& =0, \\
\mathbf{f}^{(n)} & =\left(x_{m+2}, \dot{x}_{m+2}, t_{m+2}, x_{m+3}, \dot{x}_{m+3}, t_{m+3}\right) \\
& =0, \\
\mathbf{f}^{(c)} & =\left(x_{m+3}, \dot{x}_{m+3}, t_{m+3}, x_{m+4}, \dot{x}_{m+4}, t_{m+4}\right) \\
& =0, \\
\mathbf{f}^{(a)} & =\left(x_{m+4}, \dot{x}_{m+4}, t_{m+4}, x_{m+5}, \dot{x}_{m+5}, t_{m+5}\right) \\
& =0, \\
\mathbf{f}^{(e)} & =\left(x_{m+5}, \dot{x}_{m+5}, t_{m+5}, x_{m+6}, \dot{x}_{m+6}, t_{m+6}\right) \\
& =0, \\
\mathbf{f}^{(u)} & =\left(x_{m+6}, \dot{x}_{m+6}, t_{m+6}, x_{m+7}, \dot{x}_{m+7}, t_{m+7}\right) \\
& =0 .
\end{aligned}
$$

\section{Simulations}

To illustrate the analytical conditions of passable motions, grazing motions, stick motions, sliding motions, and periodic motions, the motions of the friction-induced oscillator with an elliptic control law and the friction force acting on the mass $M$ will be demonstrated in the form of phase trajectories, time-displacement curves, time-velocity curves, and the $G$ functions responses, such as the time histories of 0th-order $G$-function and 1st-order $G$-function. The starting points of motions are described by green-solid circular symbols, and the switching points at which the oscillator may change its motion state are depicted by blue-solid circular symbols. Also the displacement curves, velocity curves, $G$-functions, and the corresponding trajectories in phase space are shown by red curves. Further, the elliptic control law curves and the velocity curves of the traveling belt are depicted by blue and green curves, respectively.

Consider a set of system parameters as $M=2 \mathrm{~kg}, \mu=0.5$, $F_{N}=20 \mathrm{~N}, Q_{0}=100 \mathrm{~N}, \Omega=2 \mathrm{rad} / \mathrm{s}, \varphi=0, B_{1}=B_{2}=5 \mathrm{~N}$, $k_{1}=4 \mathrm{~N} / \mathrm{m}, k_{2}=3 \mathrm{~N} / \mathrm{m}, \gamma_{1}=3 \mathrm{~N} \cdot \mathrm{s} / \mathrm{m}, \gamma_{2}=5 \mathrm{~N} \cdot \mathrm{s} / \mathrm{m}, V=$ $2 \mathrm{~m} / \mathrm{s}, a=8, b=6$ to demonstrate a passable motion of the mass $M$ in Figure 6. The initial conditions are selected as $x_{0}$ $=4.0 \mathrm{~m}, y_{0}=2.0 \mathrm{~m} / \mathrm{s}$, and $t_{0}=0.0743 \mathrm{~s}$. From Figure $6(\mathrm{~d})$, it can be seen that there are $F^{(2)}>0$ and $F^{(3)}>0$ at the staring time $t_{0}=0.0743 \mathrm{~s}$ or the starting point $\left(x_{0}, y_{0}\right)$ on the velocity boundary $\partial \Omega_{23}$ (i.e., $y_{0}=V$ ), which satisfies condition (39) in Theorem 8(ii). Thus the flow will move towards domain $\Omega_{2}$, as shown in Figure 6(a). Once the motion of the mass $M$ arrives at the first switching point $\left(x_{1}, y_{1}\right)=(4.315,5.097)$ on the elliptic boundary $\partial \Omega_{12}$ with time $t_{1}=0.1619 \mathrm{~s}$, the corresponding basic mapping of the mass $M$ for this process is $P_{n}$, and the velocity of the mass $M$ is greater than the 


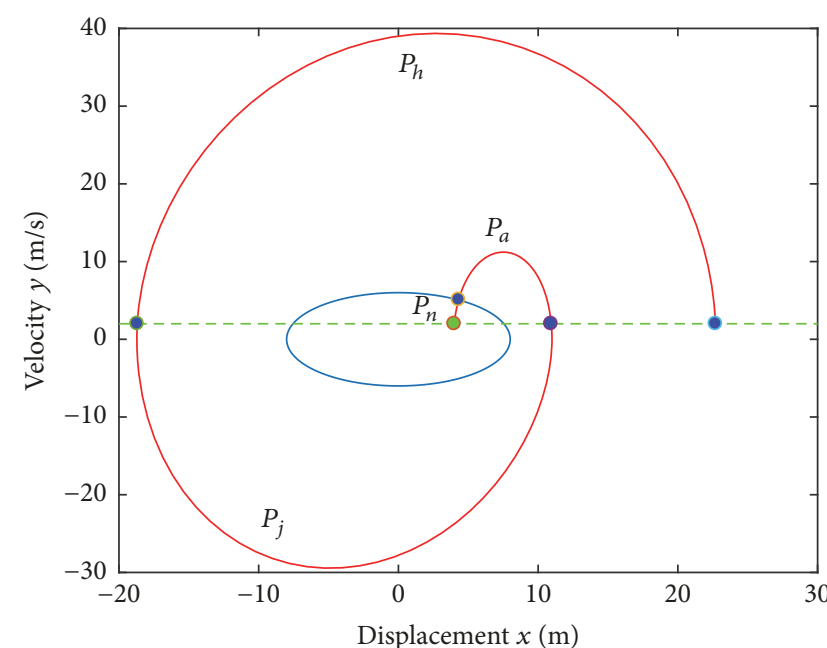

(a)

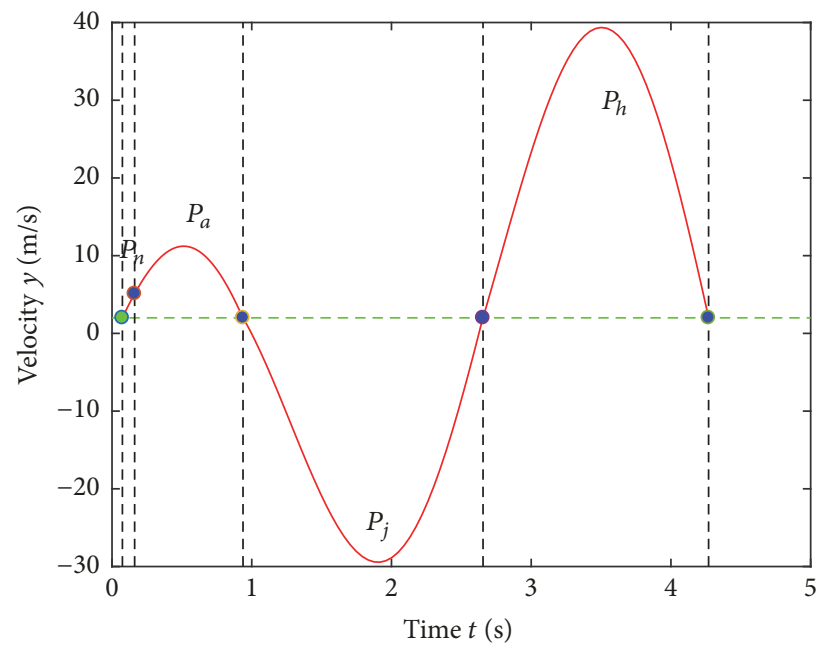

(c)

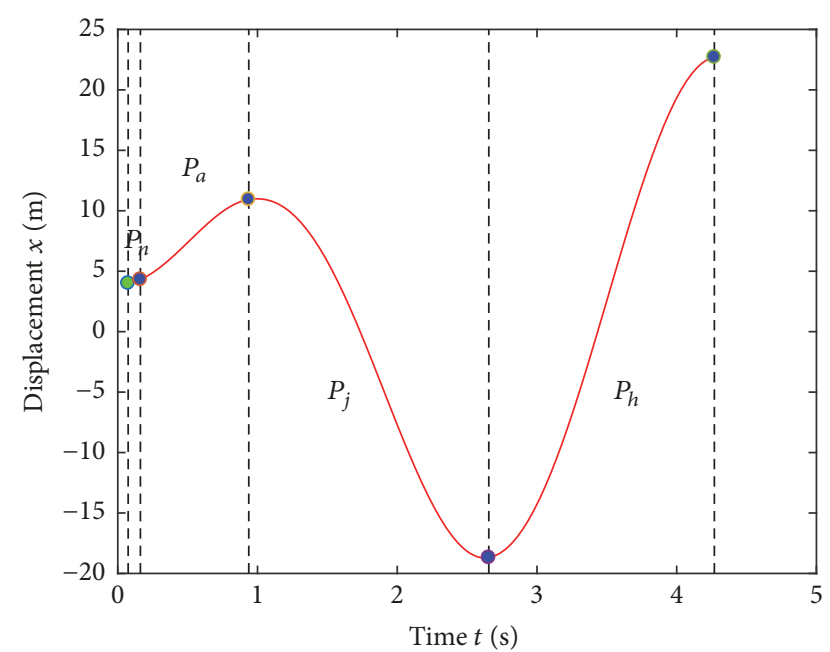

(b)

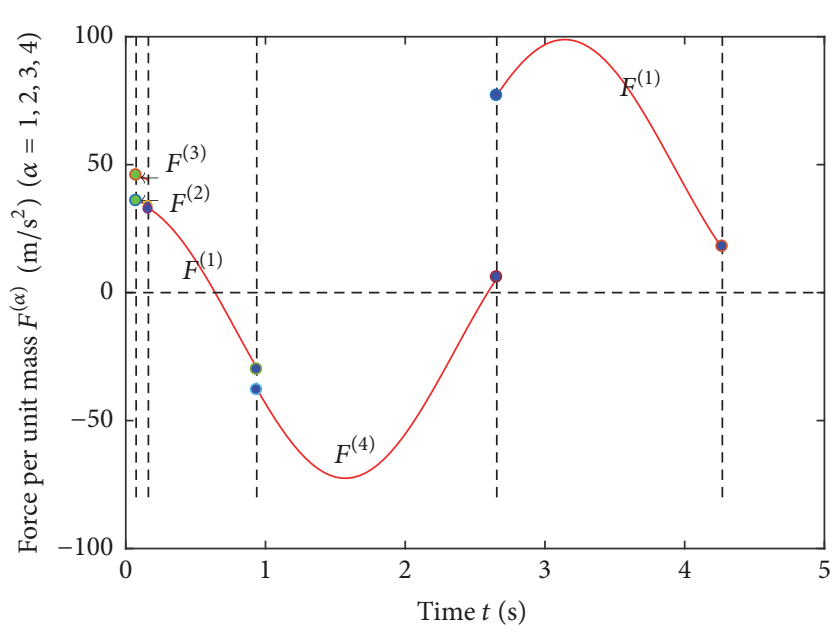

(d)

FIGURE 6: Numerical simulation of a passable motion with a mapping structure $P=P_{\text {hjan }}$ : (a) phase trajectory, (b) displacement-time history, (c) velocity-time history, and (d) force $F^{(\alpha)}$-time history $\left(M=2, \mu=0.5, F_{N}=20, Q_{0}=100, \Omega=2, \varphi=0, B_{1}=B_{2}=5, k_{1}=4, k_{2}=3\right.$, $\left.\gamma_{1}=3, \gamma_{2}=5, V=2, a=8, b=6, t_{0}=0.0743, x_{0}=4.0, y_{0}=2.0\right)$.

traveling belt's (i.e., $y_{1}>V$ ) in $\Omega_{2}$. At the first switching time $t_{1}=0.1691 \mathrm{~s}$, it is observed from Figure $6(\mathrm{~d})$ that $F^{(2)}>0$ and $F^{(1)}>0$, and thus $b^{2} x_{1} y_{1}+a^{2} y_{1} F^{(2)}>0$ and $b^{2} x_{1} y_{1}+$ $a^{2} y_{1} F^{(1)}>0$ by simple computation. The analytical condition (37) of the passable motion on the elliptic boundary $\partial \Omega_{12}$ in Theorem $8(\mathrm{i})$ is satisfied, so the flow will cross $\partial \Omega_{12}$ from domain $\Omega_{2}$ into domain $\Omega_{1}$, as shown in Figure 6(a). And the displacement and velocity response transitions are placed in Figures 6(b) and 6(c), respectively. In domain $\Omega_{1}$, the flow reaches the second switching point $\left(x_{2}, y_{2}\right)=(10.93,2)$ on the velocity boundary $\partial \Omega_{14}$ with time $t_{2}=0.9375 \mathrm{~s}$, the corresponding basic mapping of the mass $M$ for this process is $P_{a}$, and the velocity of the mass $M$ is equal to the traveling belt's (i.e., $y_{2}=V$ ). At the second switching time $t_{2}=0.9375 \mathrm{~s}$, we have $F^{(1)}<0$ and $F^{(4)}<0$ from Figure 6(d), which satisfies condition (42) in Theorem 8(iv), so the motion of the mass $M$ will cross $\partial \Omega_{14}$ from domain $\Omega_{1}$ into domain $\Omega_{4}$. The flow in domain $\Omega_{4}$ intersects the velocity boundary $\partial \Omega_{41}$ at the third switching point $\left(x_{3}, y_{3}\right)=(-18.69,2.0)$ with time $t_{3}$ $=2.6553 \mathrm{~s}$, and the corresponding basic mapping of the mass $M$ for this process is $P_{j}$, while the mass $M$ reaches the velocity boundary $\partial \Omega_{41}$ (i.e., $y_{3}=V$ ). At the third switching time $t_{3}$ $=2.6553 \mathrm{~s}$, there are $F^{(4)}>0$ and $F^{(1)}>0$, from Figure $6(\mathrm{~d})$, which satisfies condition (43) in Theorem 8 (iv), so the motion of the mass $M$ will cross $\partial \Omega_{41}$ from domain $\Omega_{4}$ into domain $\Omega_{1}$, as shown in Figure 6(a), and the motion of the mass $M$ in this process is represented by the corresponding basic mapping $P_{h}$. The corresponding trajectory in phase space, time histories of displacement, and velocity of the mass $M$ are shown in Figures 6(a)-6(c), respectively.

Consider the grazing motion of the elliptic boundary $\partial \Omega_{21}$ with parameters as $M=2 \mathrm{~kg}, \mu=0.5, F_{N}=10 \mathrm{~N}$, $Q_{0}=100 \mathrm{~N}, \Omega=5 \mathrm{rad} / \mathrm{s}, \varphi=0, B_{1}=B_{2}=0.5 \mathrm{~N}, k_{1}=$ $5 \mathrm{~N} / \mathrm{m}, k_{2}=2 \mathrm{~N} / \mathrm{m}, \gamma_{1}=1.0 \mathrm{~N} \cdot \mathrm{s} / \mathrm{m}, \gamma_{2}=0.5 \mathrm{~N} \cdot \mathrm{s} / \mathrm{m}, V=2 \mathrm{~m} / \mathrm{s}$, 
$a=8$, and $b=6$ and initial conditions as $x_{0}=-2.15 \mathrm{~m}, y_{0}$ $=3.40 \mathrm{~m} / \mathrm{s}$, and $t_{0}=0.1651 \mathrm{~s}$. The trajectory in phase plane, time histories of displacement, velocity, and $G$-functions are presented in Figure 7. From Figure 7(a), it can be seen that the mass $M$ moves freely in domain $\Omega_{2}$ at the initial point $\left(x_{0}, y_{0}\right)$. Once the motion in domain $\Omega_{2}$ arrives at the first switching point $\left(x_{1}, y_{1}\right)=(-1.415,5.898)$ on the elliptic boundary $\partial \Omega_{21}$ with $t_{1}=0.3101 \mathrm{~s}$, which is labeled by a blue circle, the velocity and displacement of the mass $M$ satisfy the conditions of $y_{1}>V$ and $x_{1}^{2} / a^{2}+y_{1}^{2} / b^{2}=1$. From Figure $7(\mathrm{~d}), G_{\partial \Omega_{12}}^{(0,2)}=G_{\partial \Omega_{21}}^{(0,2)}=b^{2} x_{1} y_{1}+a^{2} y_{1} F^{(2)}=0$ holds, and it is observed from Figure $7(\mathrm{e})$ that $G_{\partial \Omega_{12}}^{(1,2)}=G_{\partial \Omega_{21}}^{(1,2)}=$ $b^{2} x_{1} F^{(2)}+a^{2} y_{1} D F^{(2)}<0$, which satisfies condition (65) in Theorem 10(i); therefore the flow of the motion of the mass $M$ is to graze the boundary $\partial \Omega_{21}$ and then returns to domain $\Omega_{2}$, as shown in Figure 7 (a). In addition, the displacement and velocity response transitions are placed in Figures 7 (b) and $7(\mathrm{c})$, respectively.

Consider the grazing motion of the velocity boundary $\partial \Omega_{32}$ with parameters as $M=2 \mathrm{~kg}, \mu=0.5, F_{N}=10 \mathrm{~N}, Q_{0}$ $=100 \mathrm{~N}, \Omega=4 \mathrm{rad} / \mathrm{s}, \varphi=0, B_{1}=B_{2}=5 \mathrm{~N}, k_{1}=10 \mathrm{~N} / \mathrm{m}, k_{2}=$ $6 \mathrm{~N} / \mathrm{m}, \gamma_{1}=5 \mathrm{~N} \cdot \mathrm{s} / \mathrm{m}, \gamma_{2}=4 \mathrm{~N} \cdot \mathrm{s} / \mathrm{m}, V=6.73 \mathrm{~m} / \mathrm{s}, a=14$, and $b=10$ and initial conditions as $x_{0}=6.30 \mathrm{~m}, y_{0}=5.00 \mathrm{~m} / \mathrm{s}$, and $t_{0}=0.1350 \mathrm{~s}$, labeled by a green circle. The trajectory in phase plane, time histories of displacement, velocity, and $G$ functions are presented in Figure 8. According to Figure 8(a), it can be seen that the mass $M$ moves freely in domain $\Omega_{3}$ at the starting point $\left(x_{0}, y_{0}\right)$. Once the motion in domain $\Omega_{3}$ arrives at the first switching point $\left(x_{1}, y_{1}\right)=(7.25,6.73)$ on the velocity boundary $\partial \Omega_{32}$ with $t_{1}=0.2885 \mathrm{~s}$, which is labeled by a blue circle, the velocity and displacement of the mass $M$ satisfy the conditions of $y_{1}=V$ and $x_{1}^{2} / a^{2}+y_{1}^{2} / b^{2}<$ 1. From Figures $8(\mathrm{~d})$ and $8(\mathrm{e})$, we have $G_{\partial \Omega_{23}}^{(0,3)}=G_{\partial \Omega_{32}}^{(0,3)}=F^{(3)}=$ 0 and $G_{\partial \Omega_{23}}^{(1,3)}=G_{\partial \Omega_{32}}^{(1,3)}=D F^{(3)}<0$, satisfying condition (71) in Theorem 10(iv); thus the motion is to graze the $\partial \Omega_{32}$ and then goes back to domain $\Omega_{3}$, as shown in Figure 8(a). Besides, the displacement and velocity response transitions are placed in Figures 8(b) and 8(c), respectively.

Using a set of system parameters as $M=2 \mathrm{~kg}, \mu=0.5$, $F_{N}=50 \mathrm{~N}, Q_{0}=150 \mathrm{~N}, \Omega=4.8 \mathrm{rad} / \mathrm{s}, \varphi=0, B_{1}=3 \mathrm{~N}$, $B_{2}=2 \mathrm{~N}, k_{1}=4 \mathrm{~N} / \mathrm{m}, k_{2}=3 \mathrm{~N} / \mathrm{m}, \gamma_{1}=0.4 \mathrm{~N} \cdot \mathrm{s} / \mathrm{m}, \gamma_{2}=$ $0.2 \mathrm{~N} \cdot \mathrm{s} / \mathrm{m}, V=3.3 \mathrm{~m} / \mathrm{s}, a=20$, and $b=28$ and choosing the initial conditions of $t_{0}=1.9854 \mathrm{~s}, x_{0}=-11.9432 \mathrm{~m}$, and $y_{0}=17.6143 \mathrm{~m} / \mathrm{s}$, we obtain a grazing motion on the velocity boundary $\partial \Omega_{23}$ of the mass $M$, as illustrated in Figure 9. The initial point of the mass $M$ is in the domain $\Omega_{2}$, and then the mass $M$ moves freely from Figure 9(a). And the time histories of displacement and velocity of the mass $M$ are depicted in Figures 9(b) and 9(c), respectively. At time $t_{1}=2.3045 \mathrm{~s}$, the motion of the mass $M$ reaches the velocity boundary $\partial \Omega_{23}$ (i.e., $y_{1}=V$ ). For this point, as shown in Figures 9(d) and $9(\mathrm{e})$, we have $G_{\partial \Omega_{23}}^{(0,2)}=F^{(2)}=0$ and $G_{\partial \Omega_{23}}^{(1,2)}=D F^{(2)}>0$, which satisfies condition (70) in Theorem 10(iv); therefore the flow of the motion is to graze the boundary $\partial \Omega_{23}$ and then returns to domain $\Omega_{2}$, as shown in Figure 9(a).
Apply a set of system parameters as $M=1 \mathrm{~kg}, \mu=0.5$, $F_{N}=10 \mathrm{~N}, Q_{0}=10 \mathrm{~N}, \Omega=2 \mathrm{rad} / \mathrm{s}, \varphi=0, B_{1}=B_{2}=5 \mathrm{~N}$, $k_{1}=1.0 \mathrm{~N} / \mathrm{m}, k_{2}=0.5 \mathrm{~N} / \mathrm{m}, \gamma_{1}=1.0 \mathrm{~N} \cdot \mathrm{s} / \mathrm{m}, \gamma_{2}=0.5 \mathrm{~N} \cdot \mathrm{s} / \mathrm{m}$, $V=2.0 \mathrm{~m} / \mathrm{s}, a=14$, and $b=10$ to show a stick motion of the mass $M$ on the velocity boundary $\partial \Omega_{23}$ in Figure 10. The initial conditions are $t_{0}=0.12 \mathrm{~s}, x_{0}=8.5 \mathrm{~m}$, and $y_{0}=2.0 \mathrm{~m} / \mathrm{s}$, and the phase trajectory, the time histories of displacement, and velocity are plotted in Figures 10 (a)-10(c), respectively. From Figure 10(a), it can be seen that the stick motion appears at the initial point $\left(x_{0}, y_{0}\right)$, and the mass $M$ reaches the velocity boundary $\partial \Omega_{23}$ with velocity equaling $V$ and then moves together with the conveyor belt. The sticky portion is shaded by the gray color. At time $t_{1}=0.7731 \mathrm{~s}$, the blue filled circle is the vanishing point of the stick motion on the boundary $\partial \Omega_{23}$, because the motion of the mass $M$ enters domain $\Omega_{3}$ and is the free-fight motion after such a point. In order to describe the analytical conditions of stick motion on the boundary $\partial \Omega_{23}$ for the mass $M$, the time histories of $G$-functions are depicted in Figure 10(d). From Figure 10(d), it can be observed that there are $G_{\partial \Omega_{23}}^{(0,3)}=F^{(3)}>0$ and $G_{\partial \Omega_{23}}^{(0,2)}=F^{(2)}<0$ at the initial time $t_{0}=0.12 \mathrm{~s}$ on the velocity boundary $\partial \Omega_{23}$. Thus the necessary and sufficient condition (53) of the stick motion on the velocity boundary $\partial \Omega_{23}$ in Theorem 9(ii) is obtained at initial time $t_{0}=0.12 \mathrm{~s}$. It is worth noting that, within the gray portion, $G_{\partial \Omega_{23}}^{(0,3)}=F^{(3)}>0$ and $G_{\partial \Omega_{23}}^{(0,2)}=F^{(2)}<0$, so this stick motion continues until $t_{1}=0.7731 \mathrm{~s}$. But, at time $t_{1}=0.7731 \mathrm{~s}$, it can be seen that $G_{\partial \Omega_{23}}^{(0,3)}=F^{(3)}=0, G_{\partial \Omega_{23}}^{(0,2)}=F^{(2)}<0$, and $G_{\partial \Omega_{23}}^{(1,3)}=D F^{(3)}<0$ from Figures 10(d) and 10(e). Therefore, the necessary and sufficient condition (99) of the stick motion vanishing on the boundary $\partial \Omega_{23}$ in Theorem 12(ii) is satisfied. After this time $t_{1}=0.7731 \mathrm{~s}$, the motion of the mass $M$ enters the domain $\Omega_{3}$ and is free-flight motion.

Use a set of system parameters as $M=1 \mathrm{~kg}, \mu=0.5$, $F_{N}=2 \mathrm{~N}, Q_{0}=40 \mathrm{~N}, \Omega=3.2 \mathrm{rad} / \mathrm{s}, \varphi=0, B_{1}=B_{2}=2 \mathrm{~N}$, $k_{1}=15 \mathrm{~N} / \mathrm{m}, k_{2}=25 \mathrm{~N} / \mathrm{m}, \gamma_{1}=2.0 \mathrm{~N} \cdot \mathrm{s} / \mathrm{m}, \gamma_{2}=1.0 \mathrm{~N} \cdot \mathrm{s} / \mathrm{m}$, $V=0.1 \mathrm{~m} / \mathrm{s}, a=3$, and $b=2$ to show a sliding motion of the mass $M$ on the elliptic boundary $\partial \Omega_{12}$ in Figure 11 . The initial conditions are $t_{0}=0.0015 \mathrm{~s}, x_{0}=1.6947 \mathrm{~m}$, and $y_{0}$ $=1.6503 \mathrm{~m} / \mathrm{s}$, and the phase trajectory, the time histories of displacement, and velocity are plotted in Figures 11(a)-11(c), respectively. From Figure 11(a), it can be seen that the sliding motion appears at the initial point $\left(x_{0}, y_{0}\right)$ on the elliptic boundary $\partial \Omega_{12}$, and the sliding portion is shaded by the gray color. At time $t_{1}=0.1888 \mathrm{~s}$, the blue filled circle is the vanishing point of the sliding motion on the elliptic boundary $\partial \Omega_{12}$, because the motion of the mass $M$ enters domain $\Omega_{2}$ and is the free-fight motion after such a point. To describe the analytical conditions of sliding motion on the elliptic boundary $\partial \Omega_{12}$ for the mass $M$, the time histories of $G$ functions are depicted in Figure 11(d). From Figure 11(d), it can be observed that there are $G_{\partial \Omega_{12}}^{(0,1)}=b^{2} x_{0} y_{0}+a^{2} y_{0} F^{(1)}<0$ and $G_{\partial \Omega_{12}}^{(0,2)}=b^{2} x_{0} y_{0}+a^{2} y_{0} F^{(2)}>0$ at the initial time $t_{0}=$ $0.0015 \mathrm{~s}$ on the elliptic boundary $\partial \Omega_{12}$. Thus the necessary and sufficient condition (52) of the sliding motion on the elliptic boundary $\partial \Omega_{12}$ in Theorem 9(ii) is obtained at initial 


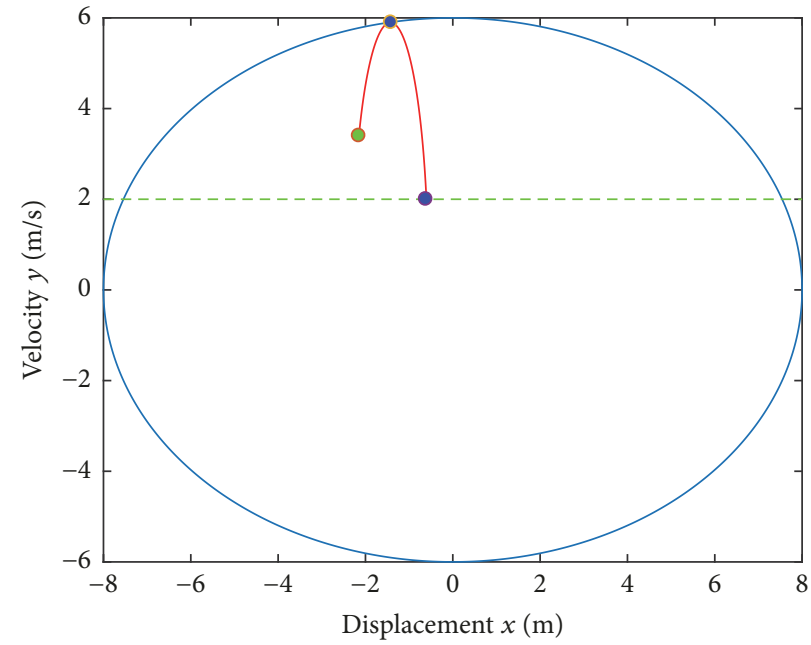

(a)

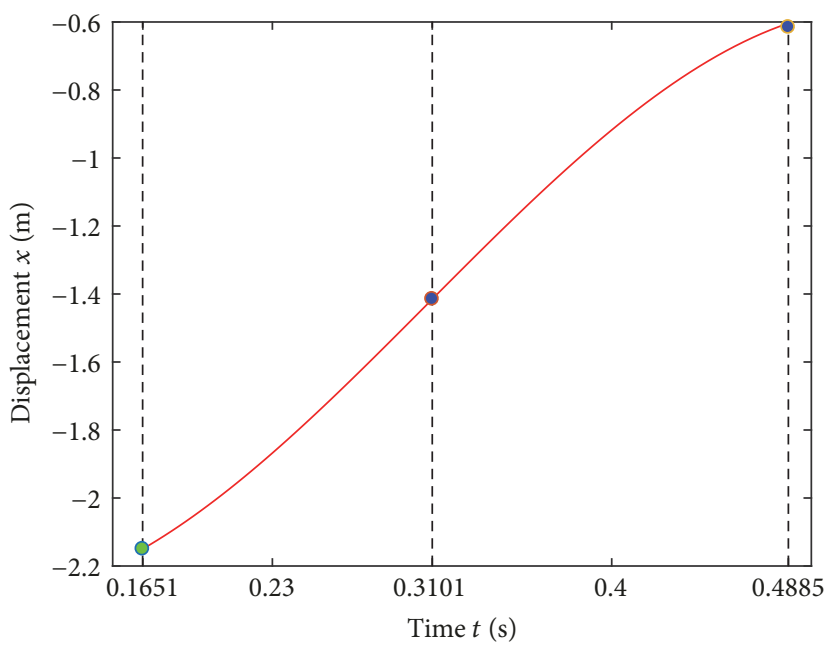

(b)

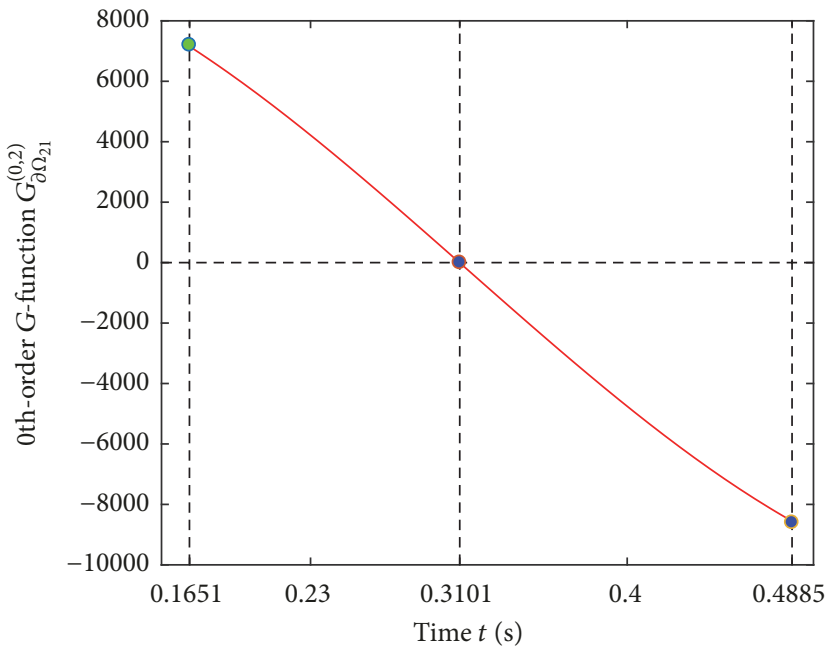

(d)

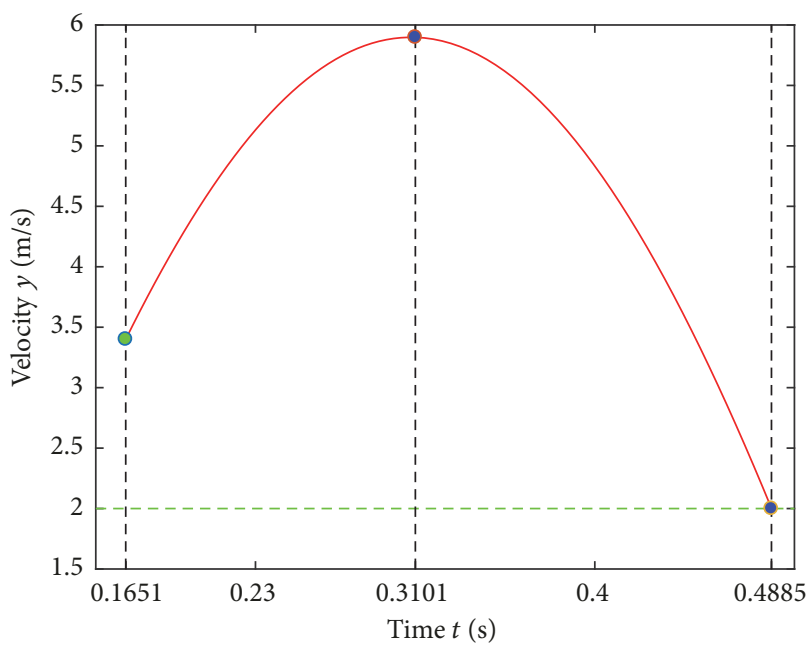

(c)

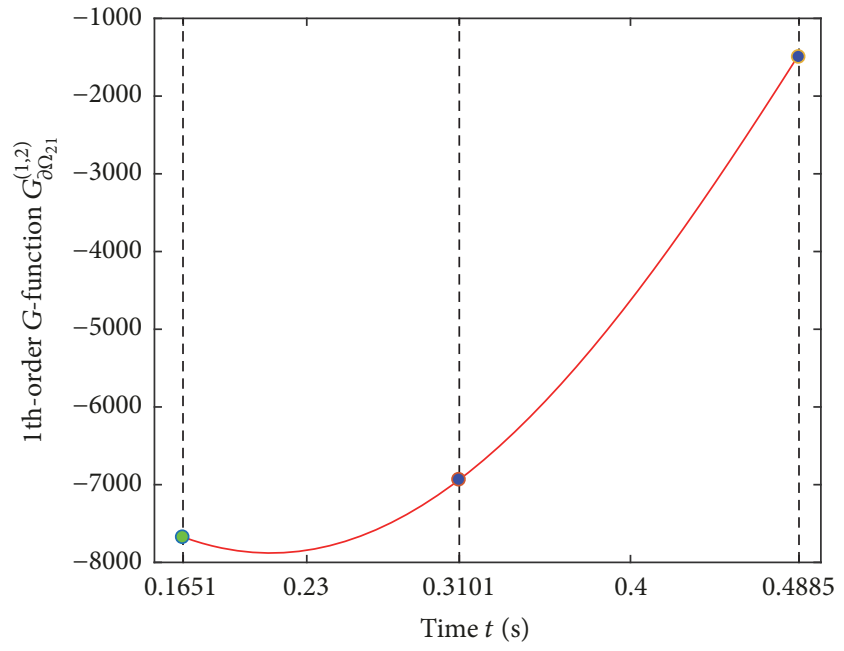

(e)

FIGURE 7: Numerical simulation of a grazing motion on elliptic switching boundary $\partial \Omega_{21}$ : (a) phase trajectory, (b) displacement-time history, (c) velocity-time history, (d) 0th-order $G$-function-time history, and (e) 1st-order $G$-function-time history $\left(M=2, \mu=0.5, F_{N}=10\right.$, $\left.Q_{0}=100, \Omega=5, \varphi=0, B_{1}=B_{2}=0.5, k_{1}=5, k_{2}=2, \gamma_{1}=1.0, \gamma_{2}=0.5, V=2, a=8, b=6, t_{0}=0.1651, x_{0}=-2.15, y_{0}=3.40\right)$. 


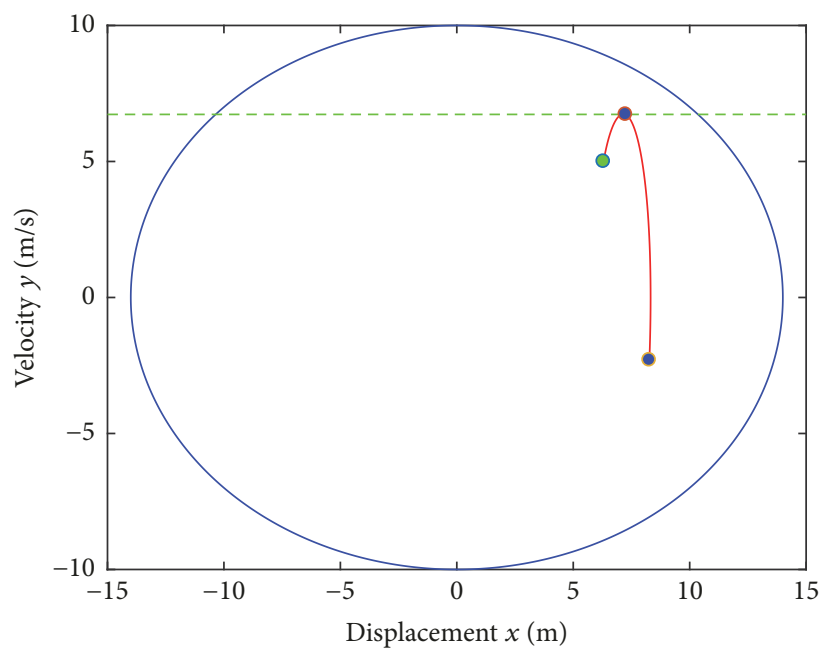

(a)

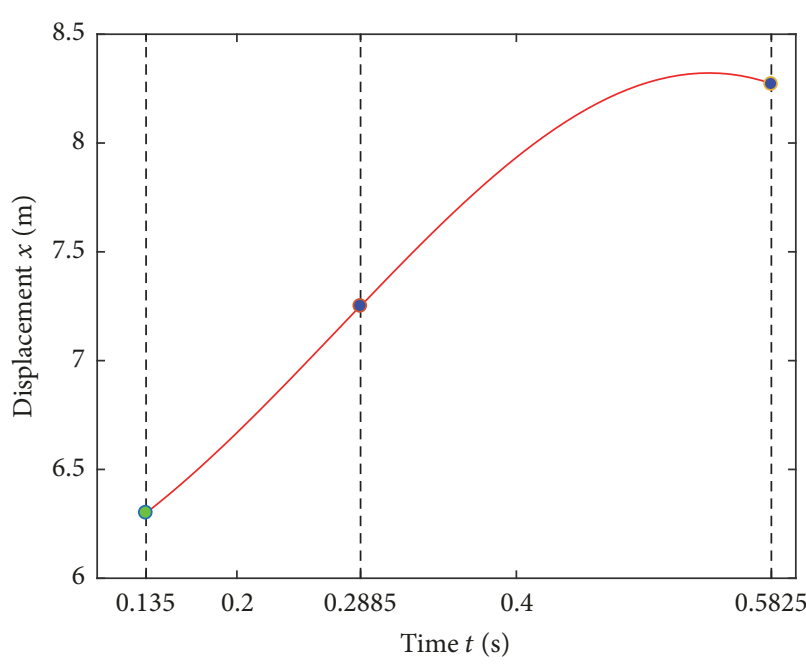

(b)

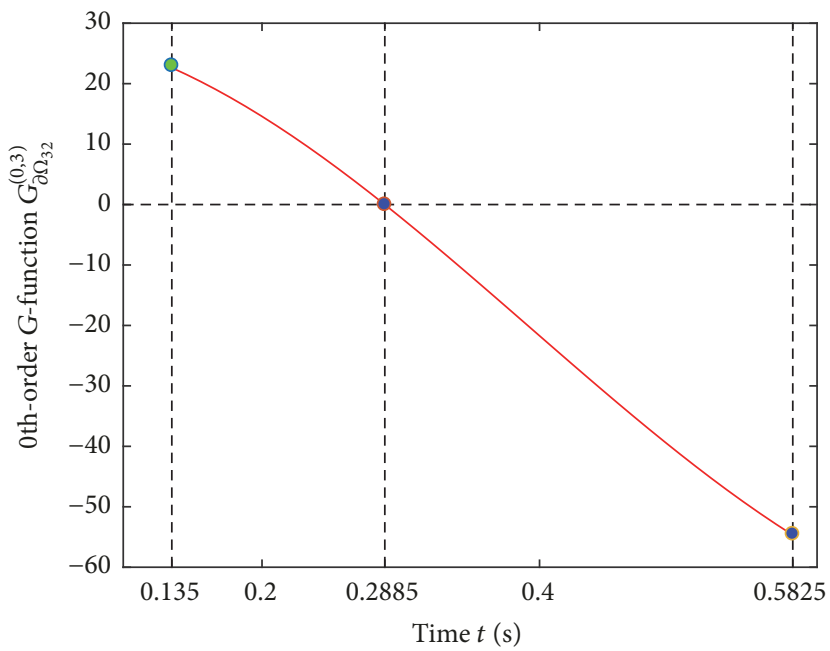

(d)

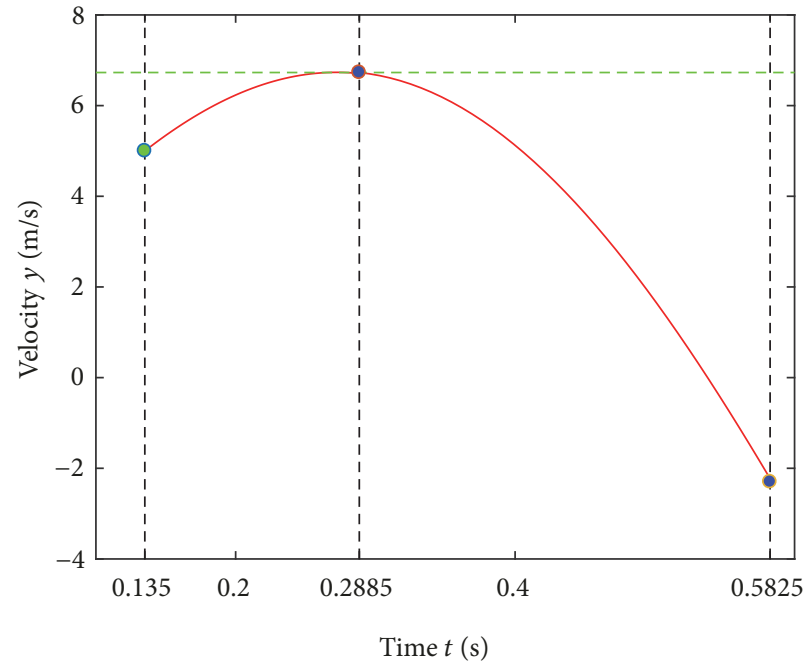

(c)

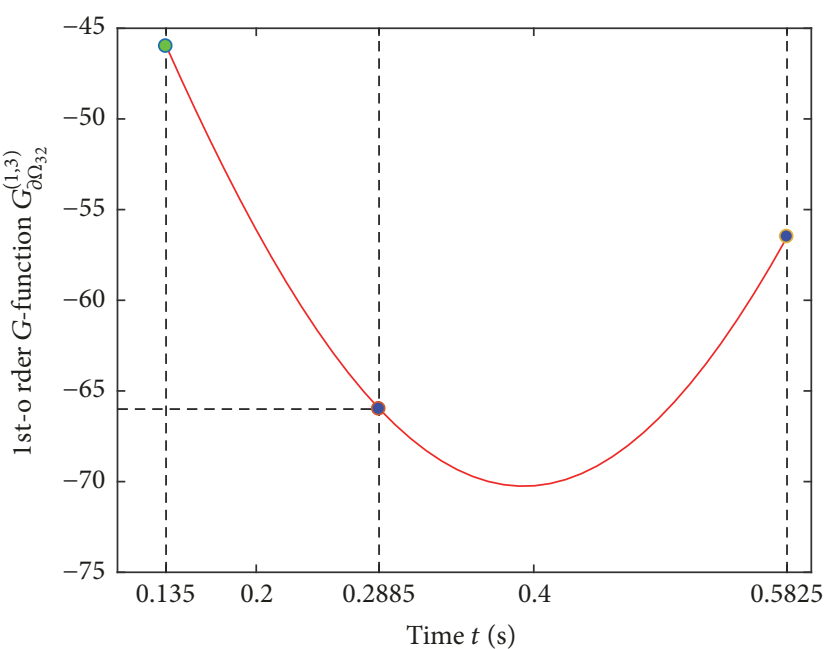

(e)

FIGURE 8: Numerical simulation of a grazing motion on velocity boundary $\partial \Omega_{32}$ : (a) phase trajectory, (b) displacement-time history, (c) velocity-time history, (d) 0 th-order $G$-function-time history, and (e) 1 st-order $G$-function-time history $\left(M=2, \mu=0.5, F_{N}=10, Q_{0}=100\right.$, $\left.\Omega=4, \varphi=0, B_{1}=B_{2}=5, k_{1}=10, k_{2}=6, \gamma_{1}=5, \gamma_{2}=4, V=6.73, a=14, b=10, t_{0}=0.1350, x_{0}=6.30, y_{0}=5.00\right)$. 


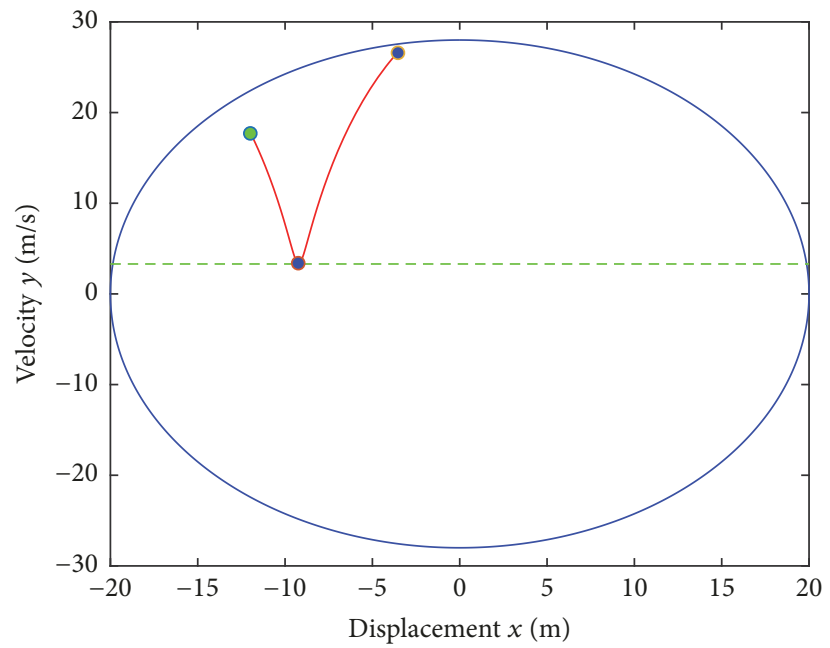

(a)

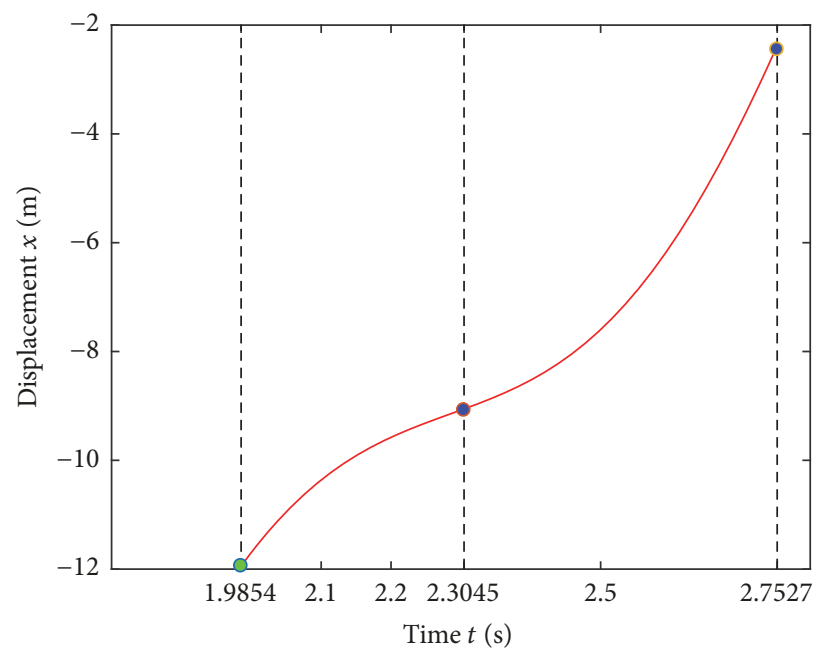

(b)

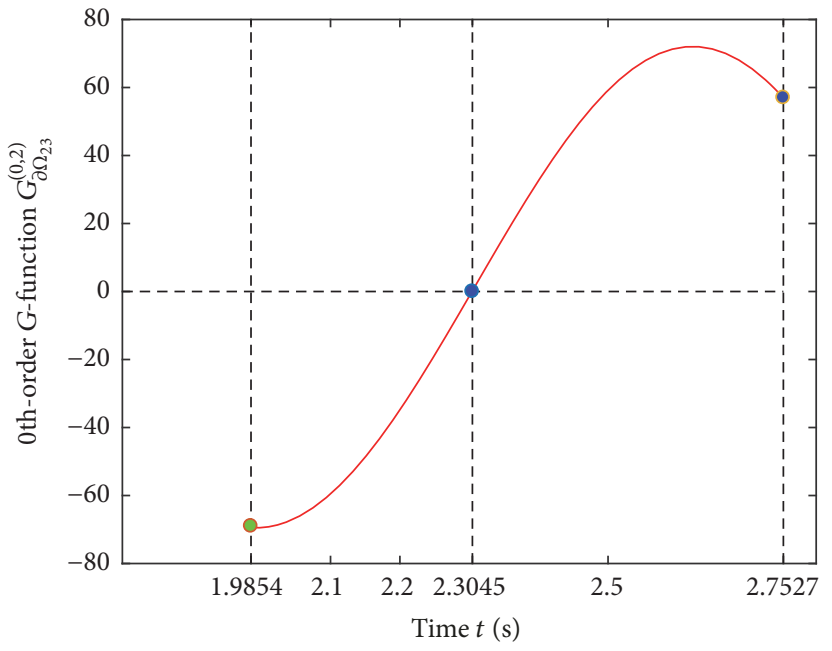

(d)

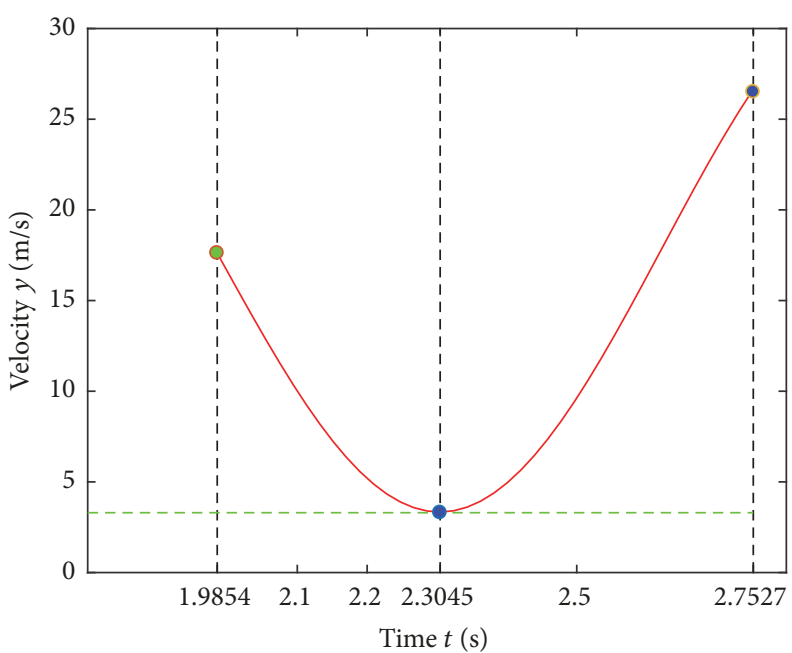

(c)

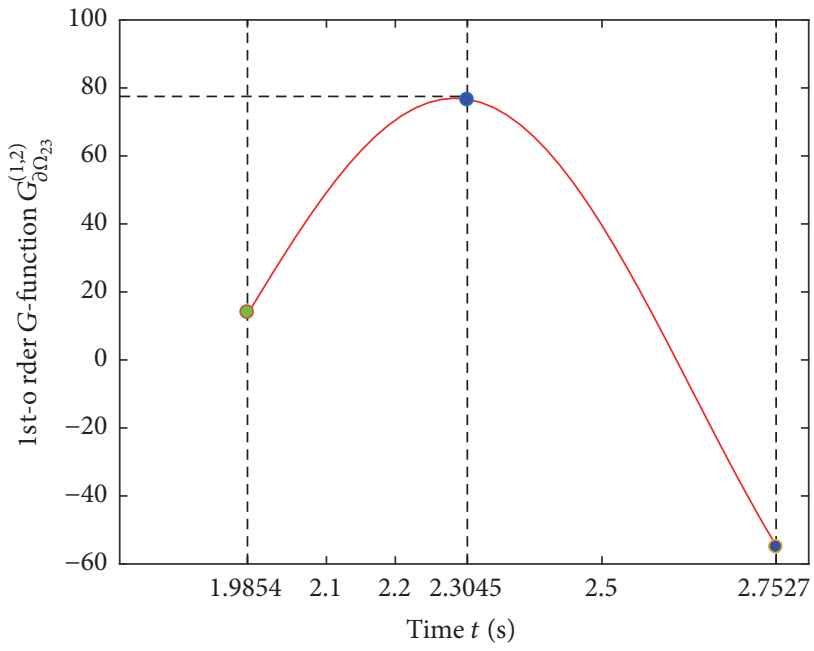

(e)

FiguRE 9: Numerical simulation of a grazing motion on velocity boundary $\partial \Omega_{23}$ : (a) phase trajectory, (b) displacement-time history, (c) velocity-time history, (d) 0th-order $G$-function-time history, (e) 1st-order $G$-function-time history $\left(M=2, \mu=0.5, F_{N}=50, Q_{0}=150\right.$, $\left.\Omega=4.8, \varphi=0, B_{1}=3, B_{2}=2, k_{1}=4, k_{2}=3, \gamma_{1}=0.4, \gamma_{2}=0.2, V=3.3, a=20, b=28, t_{0}=1.9854, x_{0}=-11.9432, y_{0}=17.6143\right)$. 


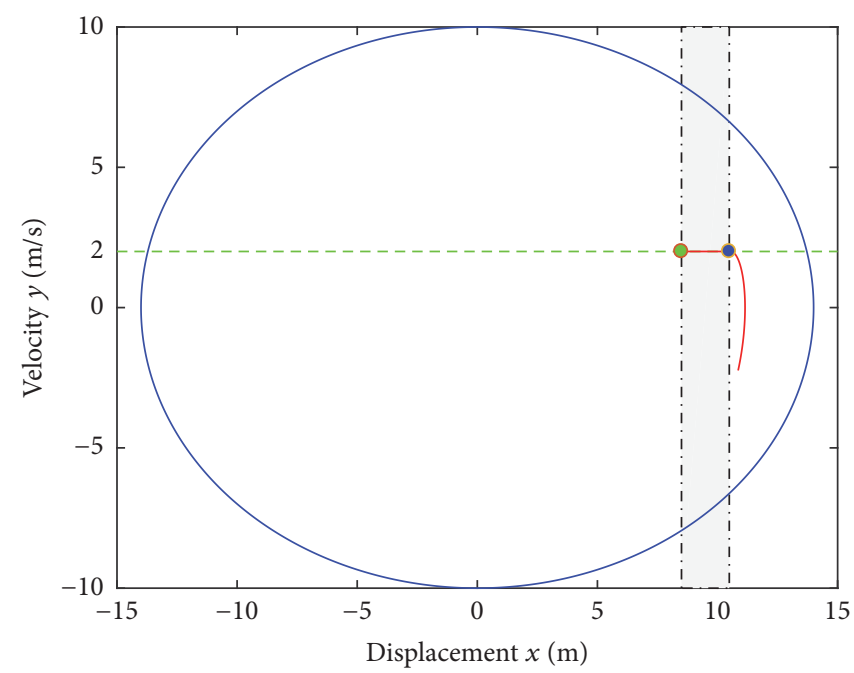

(a)

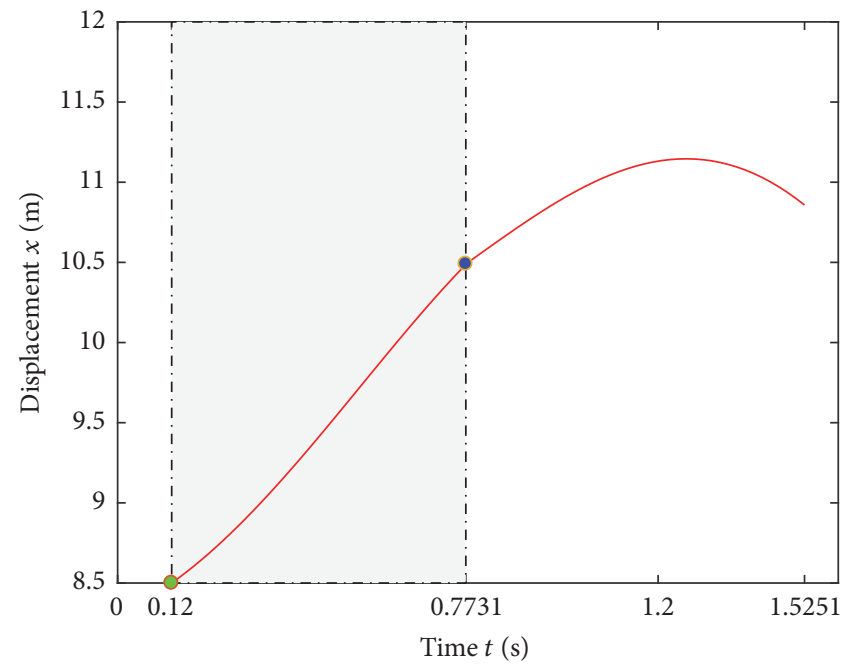

(b)

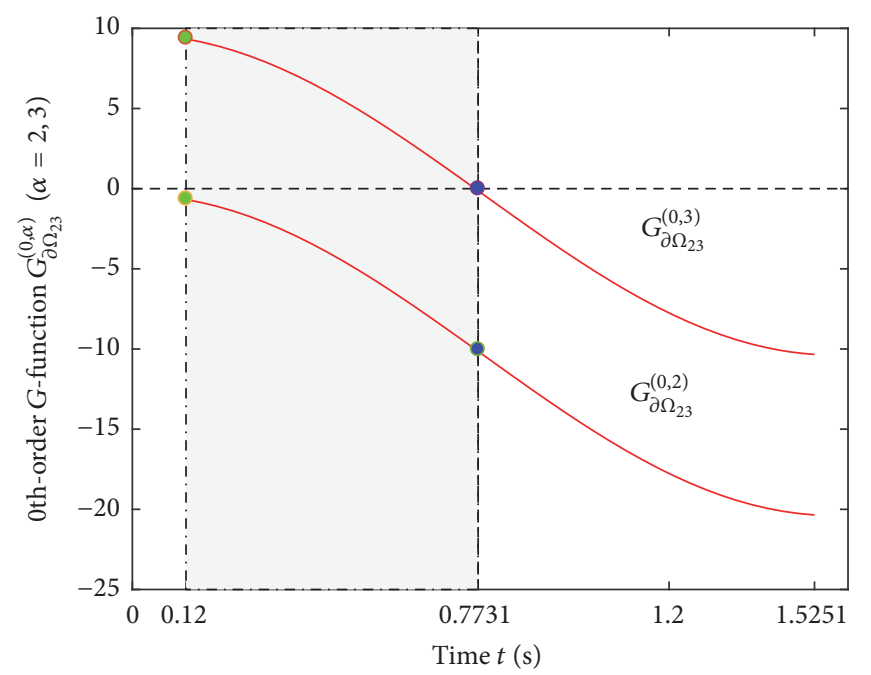

(d)

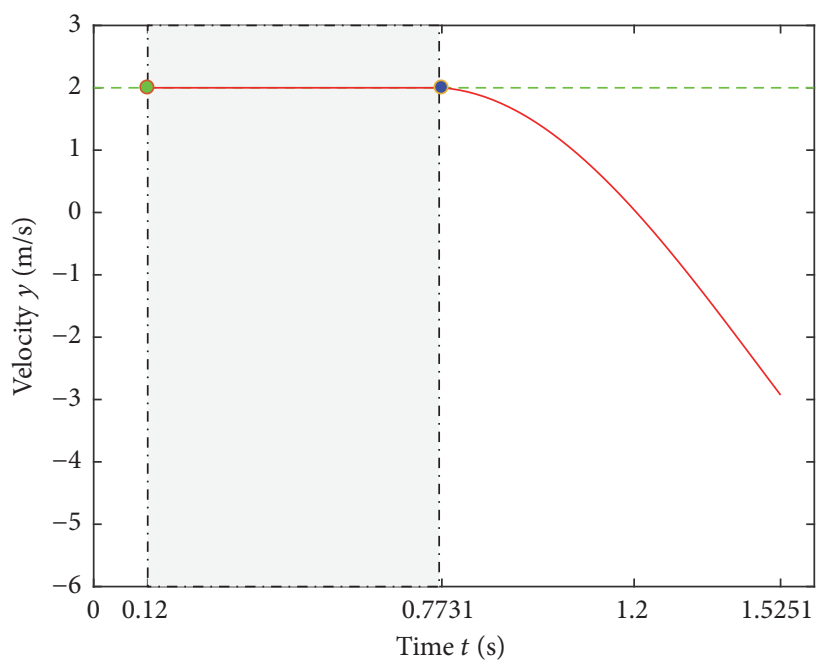

(c)

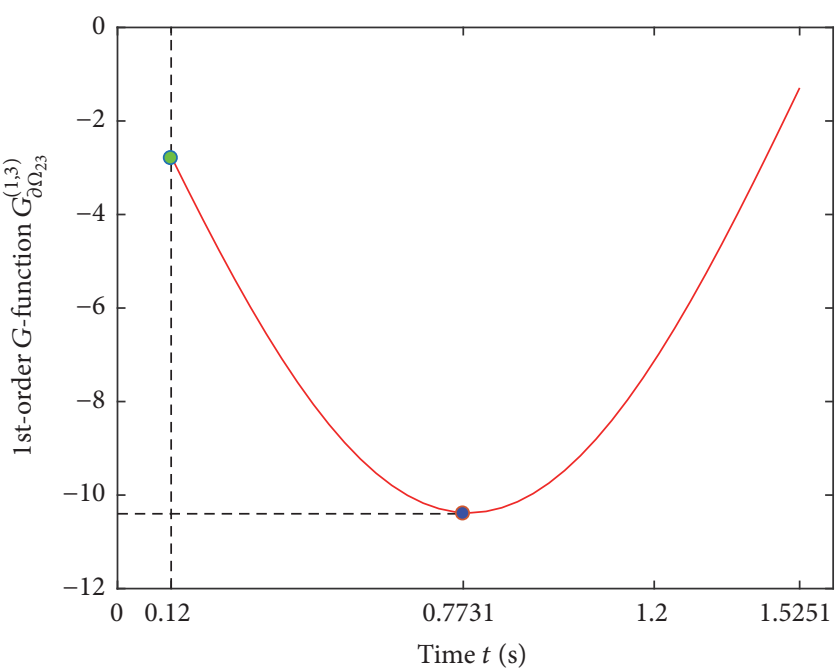

(e)

FIGURE 10: Numerical simulation of a stick motion on velocity boundary $\partial \Omega_{23}$ : (a) phase trajectory, (b) displacement-time history, (c) velocitytime history, (d) 0th-order $G$-function-time history, and (e) 1st-order $G$-function-time history $\left(M=1, \mu=0.5, F_{N}=10, Q_{0}=10, \Omega=2\right.$, $\left.\varphi=0, B_{1}=B_{2}=5, k_{1}=1.0, k_{2}=0.5, \gamma_{1}=1.0, \gamma_{2}=0.5, V=2.0, a=14, b=10, t_{0}=0.12, x_{0}=8.5, y_{0}=2.0\right)$. 


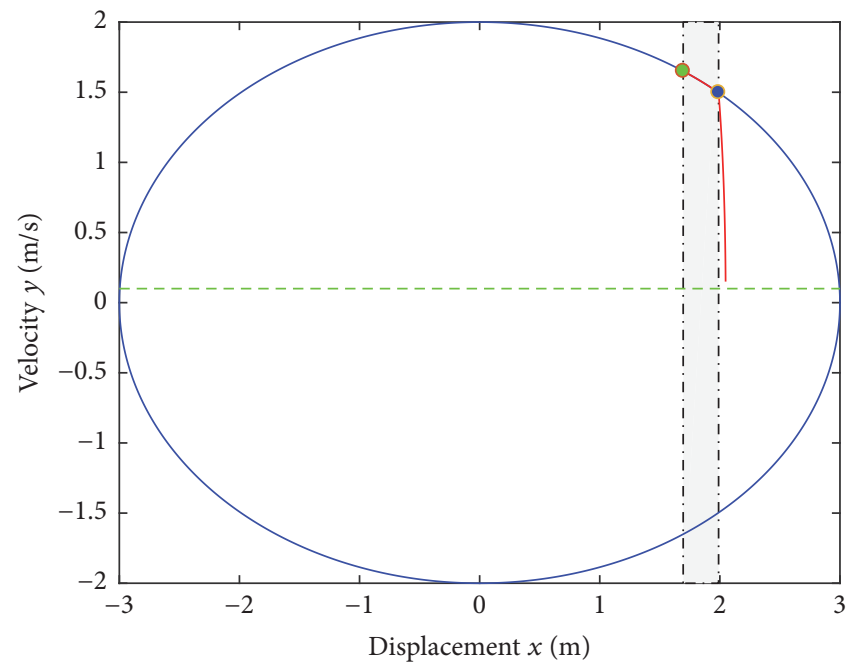

(a)

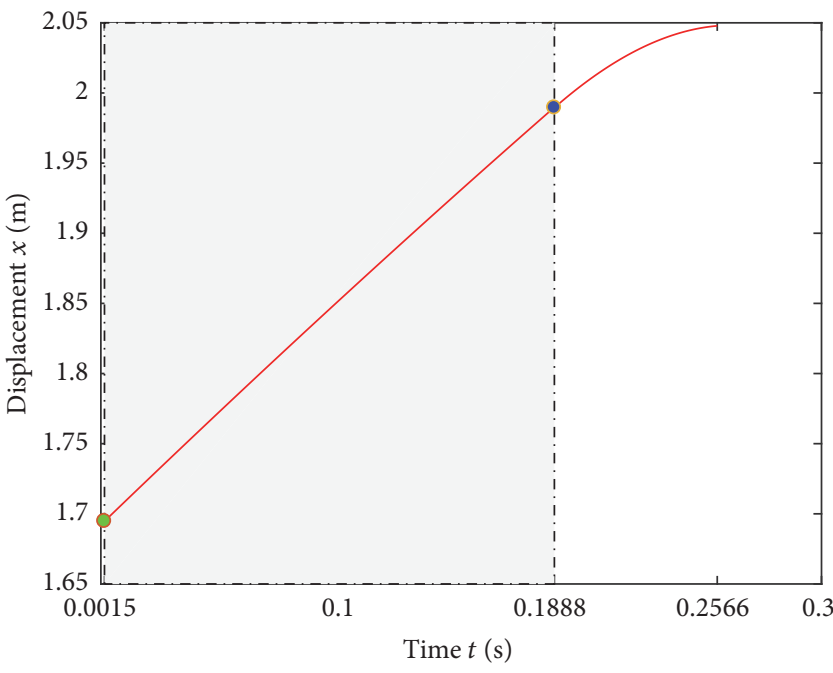

(b)

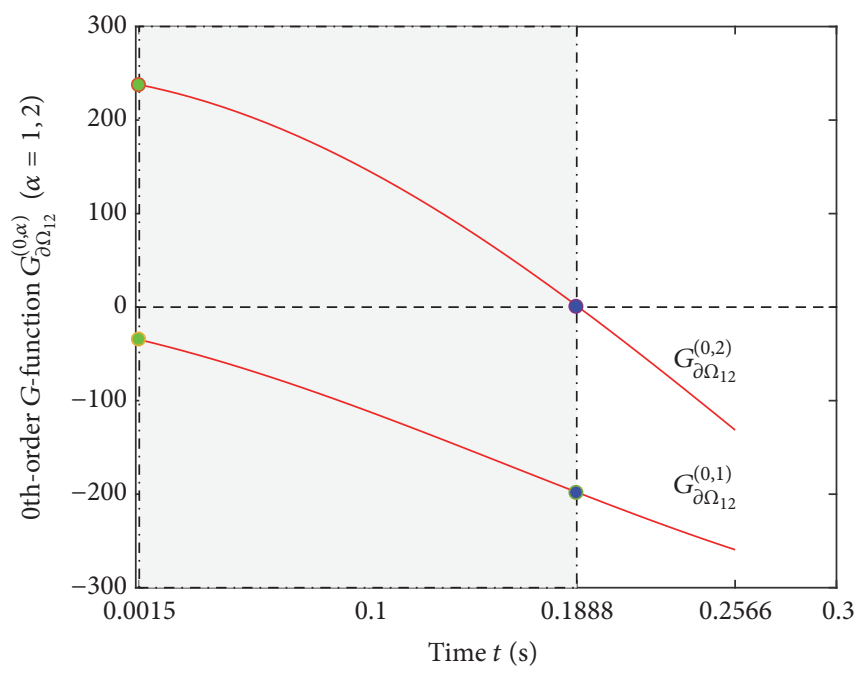

(d)

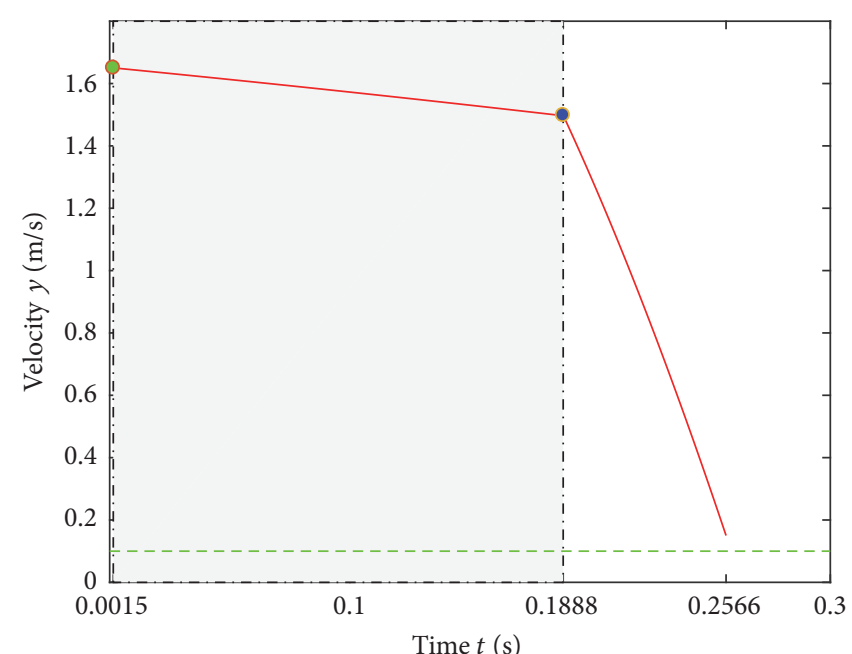

(c)

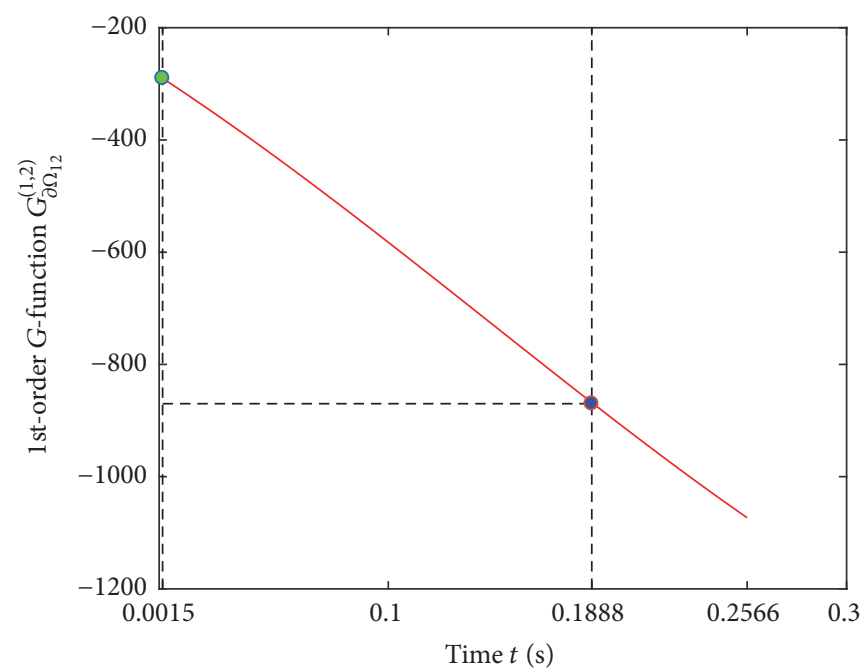

(e)

FIGURE 11: Numerical simulation of a sliding motion on elliptic boundary $\partial \Omega_{12}$ : (a) phase trajectory, (b) displacement-time history, (c) velocity-time history, (d) 0th-order $G$-function-time history, and (e) 1st-order $G$-function-time history $\left(M=1, \mu=0.5, F_{N}=2, Q_{0}=40\right.$, $\left.\Omega=3.2, \varphi=0, B_{1}=B_{2}=2, k_{1}=15, k_{2}=25, \gamma_{1}=2.0, \gamma_{2}=1.0, V=0.1, a=3, b=2, t_{0}=0.0015, x_{0}=1.6947, y_{0}=1.6503\right)$. 


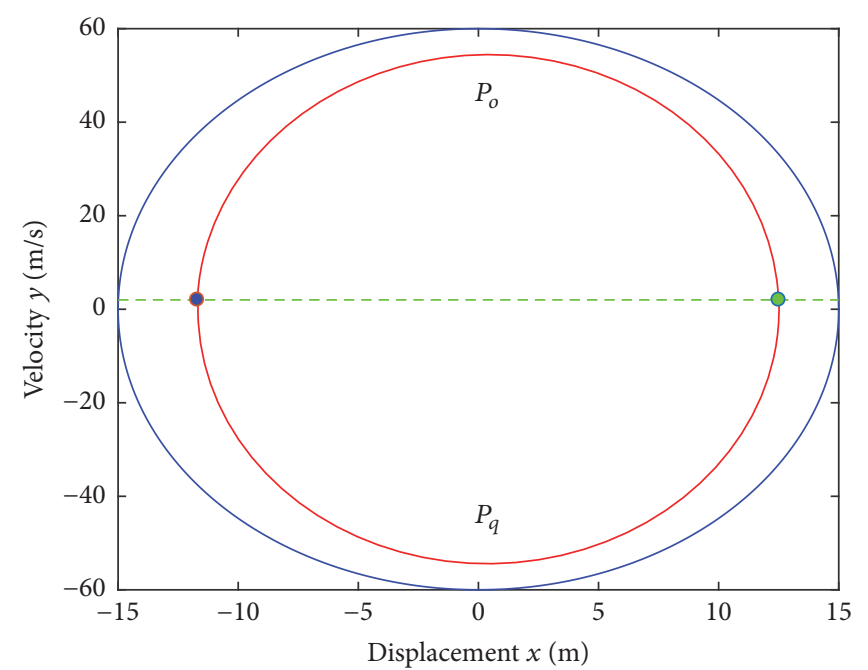

(a)

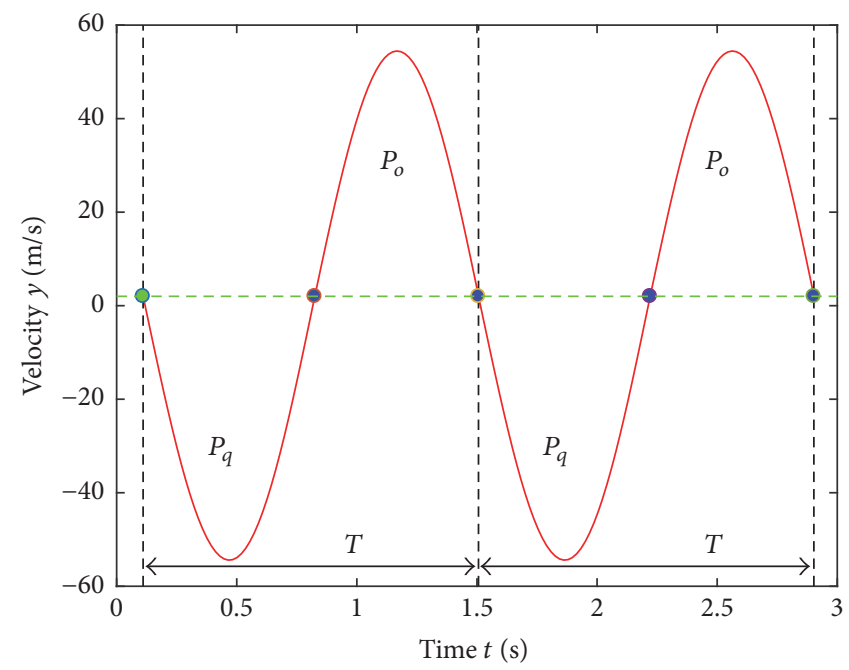

(c)

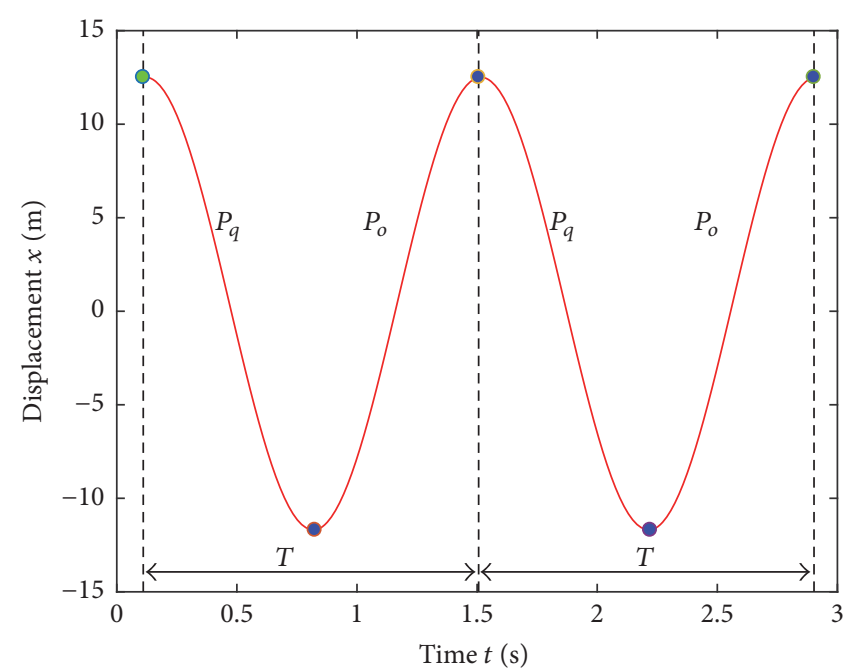

(b)

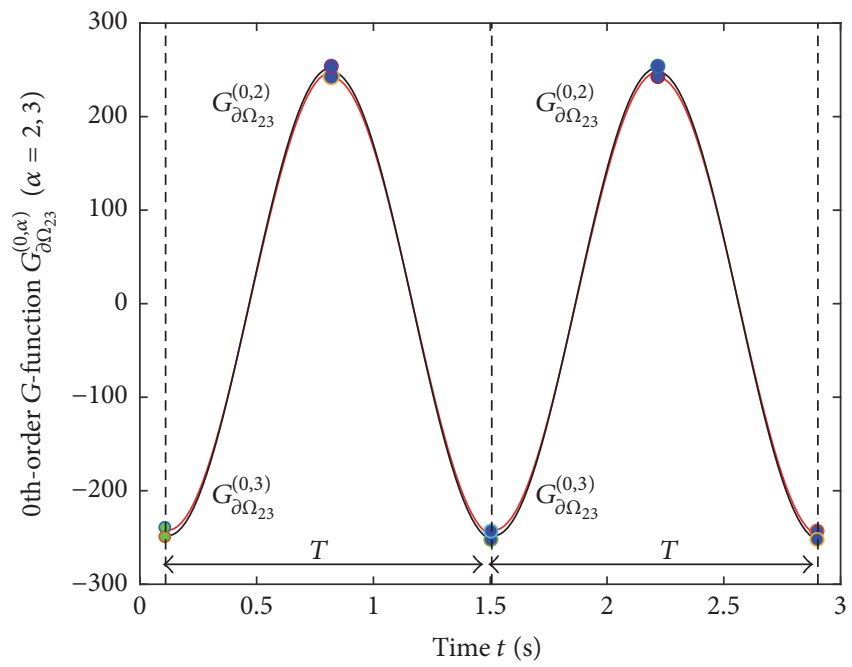

(d)

FIGURE 12: Numerical simulation of a periodic motion with a mapping structure of $P_{o q}=P_{o} \circ P_{q}$ : (a) phase trajectory, (b) displacement-time history, (c) velocity-time history, and (d) 0th-order $G$-function-time history $\left(M=1, \mu=0.5, F_{N}=6, Q_{0}=60, \Omega=4.5, \varphi=0, B_{1}=B_{2}=10\right.$, $\left.k_{1}=10, k_{2}=24.5, \gamma_{1}=1.0, \gamma_{2}=1.0, V=2.0, a=15, b=60, t_{0}=0.1102, x_{0}=12.5061, y_{0}=2.0\right)$.

time $t_{0}=0.0015 \mathrm{~s}$. It is worth noting that, within the gray portion, $G_{\partial \Omega_{12}}^{(0,1)}=b^{2} x_{0} y_{0}+a^{2} y_{0} F^{(1)}<0$ and $G_{\partial \Omega_{12}}^{(0,2)}=$ $b^{2} x_{0} y_{0}+a^{2} y_{0} F^{(2)}>0$, so this sliding motion continues until $t_{1}=0.1888 \mathrm{~s}$. But, at time $t_{1}=0.1888 \mathrm{~s}$, it can be seen that $G_{\partial \Omega_{12}}^{(0,1)}=b^{2} x_{1} y_{1}+a^{2} y_{1} F^{(1)}<0, G_{\partial \Omega_{12}}^{(0,2)}=b^{2} x_{1} y_{1}+a^{2} y_{1} F^{(2)}=0$, and $G_{\partial \Omega_{12}}^{(1,2)}=b^{2} x_{1} F^{(2)}+a^{2} y_{1} D F^{(2)}<0$ from Figures $11(\mathrm{~d})$ and 11(e). Therefore, the necessary and sufficient condition (97) of the sliding motion vanishing on the elliptic boundary $\partial \Omega_{12}$ in Theorem 12(ii) is satisfied. After this time $t_{1}=0.18888 \mathrm{~s}$, the motion of the mass $M$ enters the domain $\Omega_{2}$ and is free-flight motion.

Consider a periodic motion with a mapping structure of $P_{o q}=P_{o} \circ P_{q}$ for the mass $M$. The system parameters as $M=1 \mathrm{~kg}, \mu=0.5, F_{N}=6 \mathrm{~N}, Q_{0}=60 \mathrm{~N}, \Omega=4.5 \mathrm{rad} / \mathrm{s}$, $\varphi=0, B_{1}=B_{2}=10 \mathrm{~N}, k_{1}=10 \mathrm{~N} / \mathrm{m}, k_{2}=24.5 \mathrm{~N} / \mathrm{m}, \gamma_{1}=$
$1.0 \mathrm{~N} \cdot \mathrm{s} / \mathrm{m}, \gamma_{2}=1.0 \mathrm{~N} \cdot \mathrm{s} / \mathrm{m}, V=2.0 \mathrm{~m} / \mathrm{s}, a=15$, and $b=60$ and the initial conditions $t_{0}=0.1102 \mathrm{~s}, x_{0}=12.5061 \mathrm{~m}$, and $y_{0}=2.0 \mathrm{~m} / \mathrm{s}$ are given to demonstrate a periodic motion in Figure 12. The phase trajectory, displacement-time history, and velocity-time history of the mass $M$ are presented in Figures 12(a)-12(c), respectively. And the corresponding motions are labeled by mappings $P_{o}$ and $P_{q}$. Meanwhile, the periodicity can also be observed in displacement-time history and velocity-time history in Figures 12(b) and 12(c), and the interval between the two adjacent dashed vertical lines is one period. To understand the analytical conditions, the time histories of the $G$-functions are presented in Figure 12(d). From Figure 12(d), it can be seen that there are $G_{\partial \Omega_{23}}^{(0,2)}=$ $F^{(2)}<0$ and $G_{\partial \Omega_{23}}^{(0,3)}=F^{(3)}<0$ at the initial time $t_{0}=$ $0.1102 \mathrm{~s}$ on the velocity boundary $\partial \Omega_{23}$, which satisfies the condition (38) in Theorem 8(ii), so the flow of the periodic 


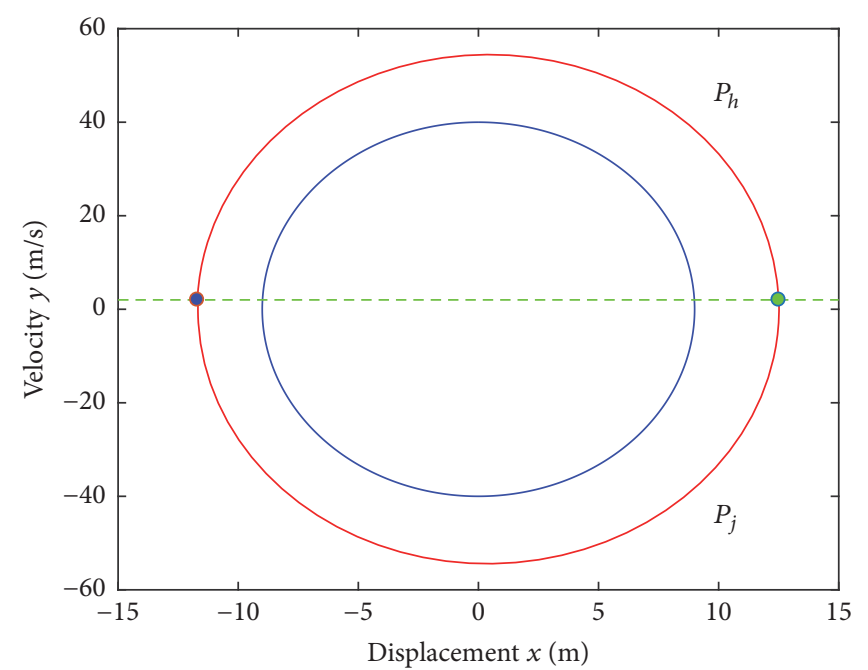

(a)

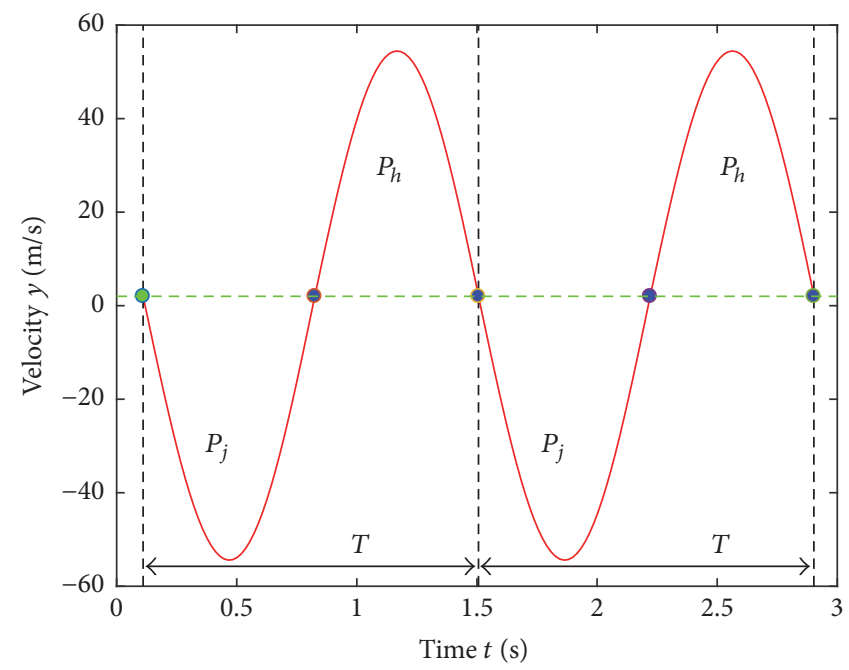

(c)

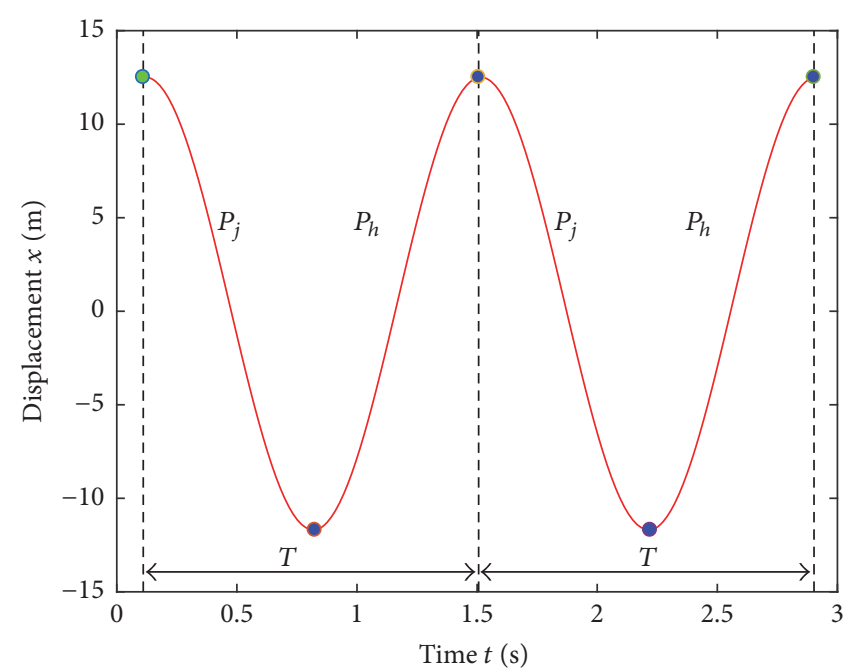

(b)

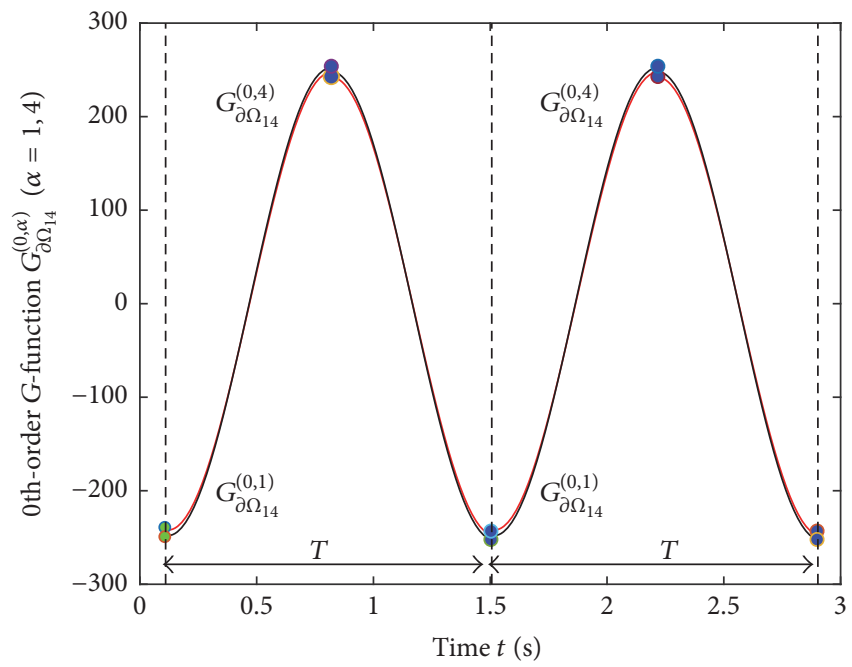

(d)

FIGURE 13: Numerical simulation of a periodic motion with a mapping structure of $P_{h j}=P_{h} \circ P_{j}$ : (a) phase trajectory, (b) displacement-time history, (c) velocity-time history, and (d) 0th-order $G$-function-time history $\left(M=1, \mu=0.5, F_{N}=6, Q_{0}=60, \Omega=4.5, \varphi=0, B_{1}=B_{2}=10\right.$, $\left.k_{1}=24.5, k_{2}=10, \gamma_{1}=1.0, \gamma_{2}=1.0, V=2.0, a=9, b=40, t_{0}=0.1102, x_{0}=12.5061, y_{0}=2.0\right)$.

motion will pass through the velocity boundary $\partial \Omega_{23}$ and enter into domain $\Omega_{3}$ from domain $\Omega_{2}$. At the first switching time $t_{1}=0.8231 \mathrm{~s}$ marked by the blue filled circle, there are $G_{\partial \Omega_{23}}^{(0,2)}=F^{(2)}>0$ and $G_{\partial \Omega_{23}}^{(0,3)}=F^{(3)}>0$ from Figure 12(d); that is, the condition (39) in Theorem 8(ii) is satisfied. Thus the flow of the periodic motion will cross the velocity boundary $\partial \Omega_{23}$ and turn into domain $\Omega_{2}$ from domain $\Omega_{3}$. Similarly, for the second switching time $t_{2}=1.5065 \mathrm{~s}$, we have $G_{\partial \Omega_{23}}^{(0,2)}=$ $F^{(2)}<0$ and $G_{\partial \Omega_{23}}^{(0,3)}=F^{(3)}<0$, which satisfies condition (38) in Theorem 8(ii); therefore the flow of the periodic motion is to pass through the velocity boundary $\partial \Omega_{23}$ and enters into domain $\Omega_{3}$ from domain $\Omega_{2}$, as shown in Figure 12(a). In the meantime, the motion of the mass $M$ in domain $\Omega_{2}$ goes back to the starting point. Therefore, the periodic motion with a mapping structure $P_{o q}=P_{o} \circ P_{q}$ under one periodic is formed.
Consider another periodic motion with a mapping structure of $P_{h j}=P_{h} \circ P_{j}$. The system parameters $M=1 \mathrm{~kg}, \mu=0.5$, $F_{N}=6 \mathrm{~N}, Q_{0}=60 \mathrm{~N}, \Omega=4.5 \mathrm{rad} / \mathrm{s}, \varphi=0, B_{1}=B_{2}=10 \mathrm{~N}$, $k_{1}=24.5 \mathrm{~N} / \mathrm{m}, k_{2}=10 \mathrm{~N} / \mathrm{m}, \gamma_{1}=1.0 \mathrm{~N} \cdot \mathrm{s} / \mathrm{m}, \gamma_{2}=1.0 \mathrm{~N} \cdot \mathrm{s} / \mathrm{m}$, $V=2.0 \mathrm{~m} / \mathrm{s}, a=9$, and $b=40$ and the starting points $x_{0}$ $=12.5061 \mathrm{~m}$ and $y_{0}=2.0 \mathrm{~m} / \mathrm{s}$ with $t_{0}=0.1102 \mathrm{~s}$ are chosen to illustrate a periodic motion of mass $M$. The phase trajectory, displacement-time history, and velocity-time history of the mass $M$ are presented in Figures 13(a)-13(c), respectively, and the corresponding motions are labeled by mappings $P_{h}$ and $P_{j}$. From the phase trajectory in Figure 13(a), it can be seen that the motion of the mass $M$ is a periodic motion. In addition, the periodicity can also be observed in displacement-time history and velocity-time history in Figures 13(b) and 13(c). For a better understanding of the analytical conditions, the time histories of the $G$-functions 


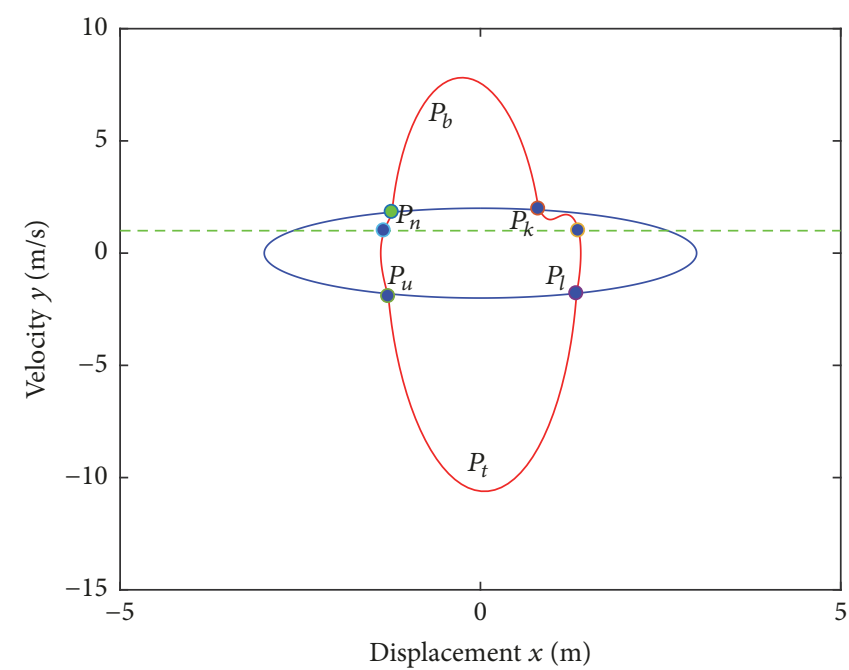

(a)

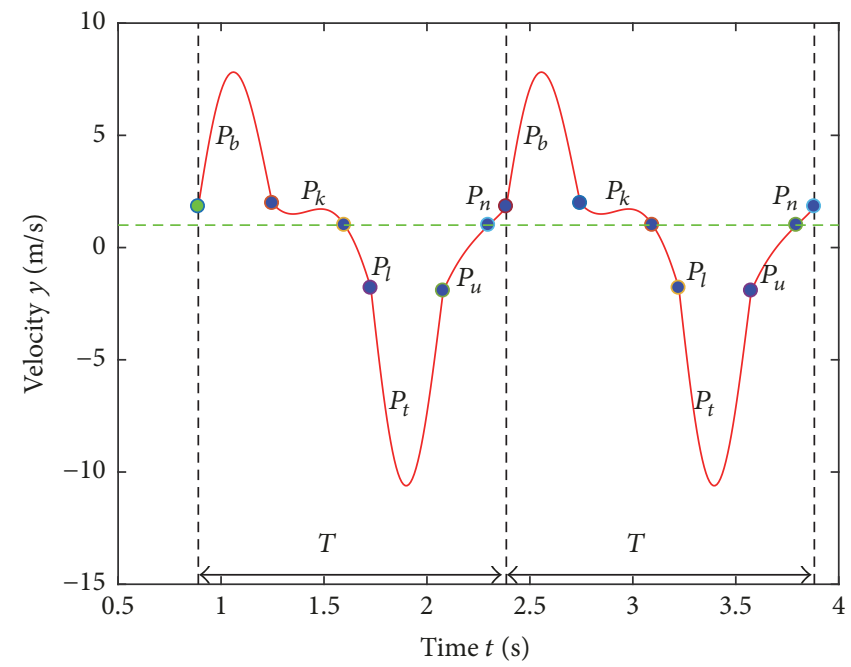

(c)

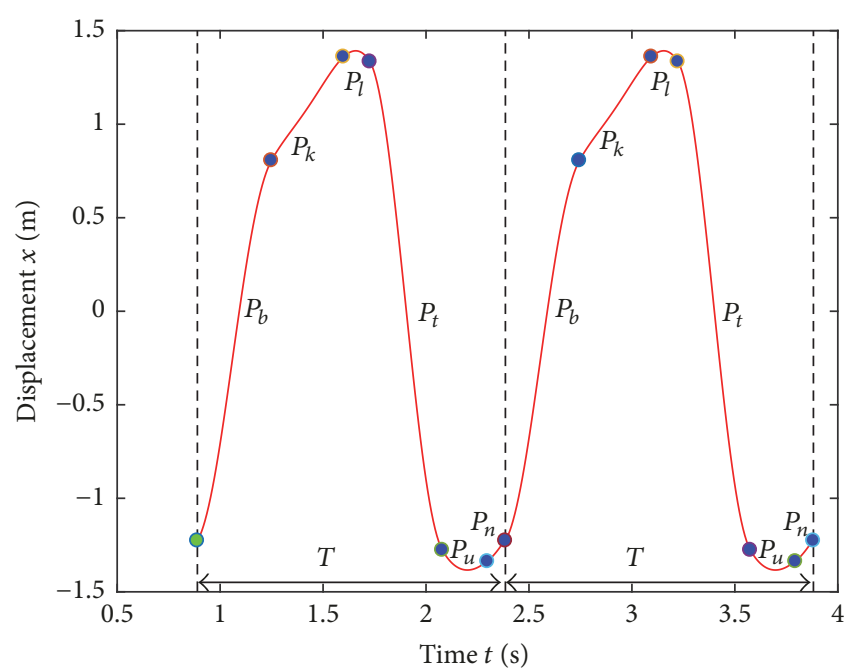

(b)

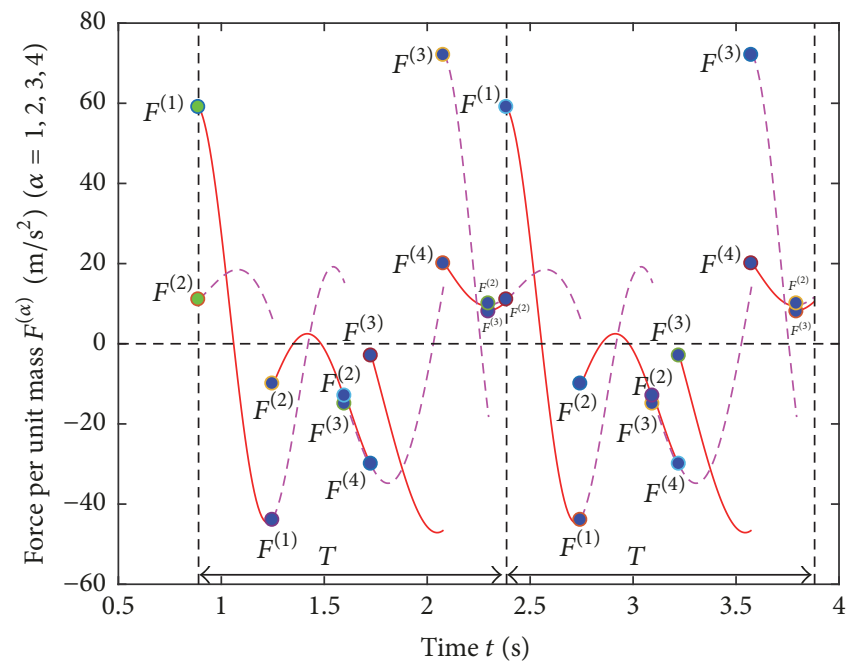

(d)

FIGURE 14: Numerical simulation of a periodic motion with a mapping structure of $P_{\text {nutlkb }}$ : (a) phase trajectory, (b) displacement-time history, (c) velocity-time history, and (d) force $F^{(\alpha)}$-time history $\left(M=1, \mu=0.5, F_{N}=1.4, Q_{0}=60, \Omega=4.2, \varphi=0, B_{1}=B_{2}=1, k_{1}=90, k_{2}=50\right.$, $\left.\gamma_{1}=2.0, \gamma_{2}=1.0, V=1.0, a=3, b=2, t_{0}=0.8895, x_{0}=-1.2269, y_{0}=1.8250\right)$.

are presented in Figure 13(d). At the initial point marked by the green filled circle, it can be observed from Figure 13(d) that $G_{\partial \Omega_{14}}^{(0,1)}=F^{(1)}<0$ and $G_{\partial \Omega_{14}}^{(0,4)}=F^{(4)}<0$, which satisfies condition (42) in Theorem 8(i); thus the flow of the periodic motion is to cross the velocity boundary $\partial \Omega_{14}$ and enters into domain $\Omega_{4}$ from domain $\Omega_{1}$. From Figure 13(d), it can be seen that there are $G_{\partial \Omega_{14}}^{(0,1)}=F^{(1)}>0$ and $G_{\partial \Omega_{14}}^{(0,4)}=F^{(4)}>0$ at the first switching point marked by the blue filled circle on the velocity boundary $\partial \Omega_{41}$ (i.e., $y_{1}=V$ ), which is satisfies condition (43) in Theorem 8(iv). So the flow of the periodic motion is to pass through the velocity boundary $\partial \Omega_{41}$ and enters into domain $\Omega_{1}$ from domain $\Omega_{4}$. Similarly, for the second switching time $t_{2}=1.5065 \mathrm{~s}$, we have $G_{\partial \Omega_{14}}^{(0,1)}=F^{(1)}<0$ and $G_{\partial \Omega_{14}}^{(0,4)}=F^{(4)}<0$; therefore the periodic motion flow is to pass through the velocity boundary $\partial \Omega_{14}$ and enters into domain $\Omega_{4}$ from domain $\Omega_{1}$, as shown in Figure 13(a). At the same time, the motion of the mass $M$ in domain $\Omega_{1}$ goes back to the starting point. Therefore, the periodic motion with a mapping structure $P_{h j}=P_{h} \circ P_{j}$ under one period is formed.

Consider another periodic motion with a mapping structure of $P_{\text {nutlkb }}$ with parameters as $M=1 \mathrm{~kg}, \mu=0.5, F_{N}=1.4 \mathrm{~N}$, $Q_{0}=60 \mathrm{~N}, \Omega=4.2 \mathrm{rad} / \mathrm{s}, \varphi=0, B_{1}=B_{2}=1 \mathrm{~N}, k_{1}=90 \mathrm{~N} / \mathrm{m}$, $k_{2}=50 \mathrm{~N} / \mathrm{m}, \gamma_{1}=2.0 \mathrm{~N} \cdot \mathrm{s} / \mathrm{m}, \gamma_{2}=1.0 \mathrm{~N} \cdot \mathrm{s} / \mathrm{m}, V=1.0 \mathrm{~m} / \mathrm{s}$, $a=3, b=2$. The initial conditions are adopted on the elliptic boundary $\partial \Omega_{12}$ as $x_{0}=-1.2269 \mathrm{~m}, y_{0}=1.8250 \mathrm{~m} / \mathrm{s}$ with $t_{0}$ $=0.8895 \mathrm{~s}$. The trajectory in phase plane, time histories of displacement, velocity, and forces are presented in Figure 14. And the starting points of this motion are labeled by the green filled circles in Figures 14(a)-14(c). It is observed from Figure 14(d) that there are $F^{(1)}>0$ and $F^{(2)}>0$; thus $b^{2} x_{0} y_{0}+$ $a^{2} y_{0} F^{(1)}>0$ and $b^{2} x_{0} y_{0}+a^{2} y_{0} F^{(2)}>0$ hold by simple 
calculation, which satisfies condition (37) in Theorem 8(i). Therefore the mass $M$ will move towards domain $\Omega_{1}$, as shown in Figure 14(a). At this time, the corresponding basic mapping of the mass $M$ is $P_{b}$. In addition, the red solid curves and magenta dotted curves denote the real and imaginary normal vector fields in Figure 14(d), respectively. For the first switching time $t_{1}=1.2491 \mathrm{~s}$, it is observed from Figure $14(\mathrm{~d})$ that $F^{(1)}<0$ and $F^{(2)}<0$, and thus $b^{2} x_{1} y_{1}+a^{2} y_{1} F^{(1)}<0$ and $b^{2} x_{1} y_{1}+a^{2} y_{1} F^{(2)}<0$ through simple computation; that is, condition (36) in Theorem $8(i)$ is satisfied. Thus the flow of the periodic motion will pass through the elliptic boundary $\partial \Omega_{12}$ and enter into domain $\Omega_{2}$ from domain $\Omega_{1}$. At this time, the corresponding basic mapping of the mass $M$ is $P_{k}$. And, at the second switching time $t_{2}=1.5991 \mathrm{~s}$, from Figure $14(\mathrm{~d})$, we have $F^{(2)}<0$ and $F^{(3)}<0$; in other words, condition (38) in Theorem 8(ii) is satisfied. Hence the flow of the periodic motion will cross the velocity boundary $\partial \Omega_{23}$ and enter into domain $\Omega_{3}$ from domain $\Omega_{2}$; the corresponding basic mapping of the mass $M$ for this process is $P_{l}$, as shown in Figure 14(a). The motion of the mass $M$ in domain $\Omega_{3}$ reaches the third switching point $\left(x_{3}, y_{3}\right)=(1.3340,-1.8010)$ on the elliptic boundary $\partial \Omega_{34}$ with time $t_{3}=1.7275 \mathrm{~s}$, and it is observed that $F^{(3)}<0$ and $F^{(4)}<0$ from Figure $14(\mathrm{~d})$ and $b^{2} x_{3} y_{3}+a^{2} y_{3} F^{(3)}>0$ and $b^{2} x_{3} y_{3}+a^{2} y_{3} F^{(4)}>0$ by simple computation, which satisfies condition (40) in Theorem 8(iii). Thus the flow of the periodic motion will pass through the elliptic boundary $\partial \Omega_{34}$ and turn into domain $\Omega_{4}$ from domain $\Omega_{3}$; the corresponding basic mapping of the mass $M$ for this process is $P_{t}$, as shown in Figure 14(a). Once the motion in domain $\Omega_{4}$ arrives at the fourth switching point $\left(x_{4}, y_{4}\right)=(-1.2770,-1.9270)$ on the elliptic boundary $\partial \Omega_{43}$ with time $t_{4}=2.0792 \mathrm{~s}$, we have $F^{(3)}>0$ and $F^{(4)}>0$ from Figure $14(\mathrm{~d})$ and $b^{2} x_{4} y_{4}+$ $a^{2} y_{4} F^{(3)}<0$ and $b^{2} x_{4} y_{4}+a^{2} y_{4} F^{(4)}<0$ by simple computation; therefore condition (41) in Theorem 8(iii) is satisfied. So the flow of the periodic motion will pass through the elliptic boundary $\partial \Omega_{43}$ and enter into domain $\Omega_{3}$ from domain $\Omega_{4}$, and the corresponding basic mapping of the mass $M$ for this process is $P_{u}$, as shown in Figure 14(a). The flow in domain $\Omega_{3}$ intersects the velocity boundary $\partial \Omega_{32}$ at the fifth switching point $\left(x_{5}, y_{5}\right)=(-1.3378,1.0)$ with time $t_{5}$ $=2.2985 \mathrm{~s}$; inequalities $F^{(3)}>0$ and $F^{(2)}>0$ follow from Figure 14(d), which satisfies condition (39) in Theorem 8(ii), so the motion of the mass $M$ will cross $\partial \Omega_{32}$ from domain $\Omega_{3}$ into domain $\Omega_{2}$, as shown in Figure 14(a). At this time, the corresponding basic mapping of the mass $M$ is $P_{n}$. And the flow of motion of the mass $M$ in domain $\Omega_{2}$ goes back to the starting point $\left(x_{0}, y_{0}\right)$ at time $t_{6}=2.3855 \mathrm{~s}$, as shown in Figure 14(a). Therefore, the periodic motion with a mapping structure $P_{n u t l k b}$ under one period is formed.

Consider another periodic motion with a mapping structure of $P_{\text {acgfse }}$ with parameters as $M=1 \mathrm{~kg}, \mu=0.5, F_{N}=$ $6 \mathrm{~N}, Q_{0}=60 \mathrm{~N}, \Omega=4.5 \mathrm{rad} / \mathrm{s}, \varphi=0, B_{1}=B_{2}=10 \mathrm{~N}, k_{1}=$ $24.5 \mathrm{~N} / \mathrm{m}, k_{2}=10 \mathrm{~N} / \mathrm{m}, \gamma_{1}=1.0 \mathrm{~N} \cdot \mathrm{s} / \mathrm{m}, \gamma_{2}=1.0 \mathrm{~N} \cdot \mathrm{s} / \mathrm{m}, V=$ $2.0 \mathrm{~m} / \mathrm{s}, a=10$, and $b=60$. The initial conditions are adopted on the velocity boundary $\partial \Omega_{14}$ as $x_{0}=13.0205 \mathrm{~m}, y_{0}=$ $2.0 \mathrm{~m} / \mathrm{s}$ with $t_{0}=0.1262 \mathrm{~s}$. The trajectory in phase plane, time histories of displacement, velocity, and forces are presented in Figure 15. And the starting points of this motion are labeled by the green filled circles in Figures 15(a)-15(c). It is observed from Figure $15(\mathrm{~d})$ that there are $F^{(1)}<0$ and $F^{(4)}<0$, which satisfies condition (42) in Theorem 8(iv). Therefore the mass $M$ will move towards domain $\Omega_{4}$, as shown in Figure 14(a). At this time, the corresponding basic mapping of the mass $M$ is $P_{e}$. In addition, the red solid curves and magenta dotted curves denote the real and imaginary normal vector fields in Figure 15(d), respectively. Once the motion of the mass $M$ arrives at the first switching point $\left(x_{1}, y_{1}\right)=$ $(4.0871,-54.7601)$ on the elliptic boundary $\partial \Omega_{43}$ with time $t_{1}=0.4155 \mathrm{~s}$, it is observed from Figure $15(\mathrm{~d})$ that $F^{(4)}>0$ and $F^{(3)}>0$, and thus $b^{2} x_{1} y_{1}+a^{2} y_{1} F^{(4)}<0$ and $b^{2} x_{1} y_{1}+$ $a^{2} y_{1} F^{(3)}<0$ through simple computation; that is, condition (41) in Theorem 8(iii) is satisfied. Thus the flow of the periodic motion will pass through the elliptic boundary $\partial \Omega_{43}$ and enter into domain $\Omega_{3}$ from domain $\Omega_{4}$. At this time, the corresponding basic mapping of the mass $M$ is $P_{s}$. And at the second switching point $\left(x_{2}, y_{2}\right)=(-4.5141,-53.6501)$ on the elliptic boundary $\partial \Omega_{34}$ with time $t_{2}=0.5722 \mathrm{~s}$, we have $F^{(3)}<$ 0 and $F^{(4)}<0$ from Figure 15(d) and $b^{2} x_{2} y_{2}+a^{2} y_{2} F^{(3)}>0$ and $b^{2} x_{2} y_{2}+a^{2} y_{2} F^{(4)}>0$ by simple computation, which satisfies condition (40) in Theorem 8(iii). Hence the flow of the periodic motion will cross the boundary $\partial \Omega_{34}$ and enter into domain $\Omega_{4}$ from domain $\Omega_{3}$, and the corresponding basic mapping of the mass $M$ for this time is $P_{f}$, as shown in Figure 15(a). The motion of the mass $M$ in domain $\Omega_{4}$ reaches the third switching point $\left(x_{3}, y_{3}\right)=(-12.3805,2.0)$ on the velocity boundary $\partial \Omega_{41}$ with time $t_{3}=0.8386 \mathrm{~s}$; it is observed from Figure $15(\mathrm{~d})$ that $F^{(4)}>0$ and $F^{(1)}>0$, which satisfies condition (43) in Theorem 8 (iv). Thus the flow of the periodic motion will pass through the boundary $\partial \Omega_{41}$ and turn into domain $\Omega_{1}$ from domain $\Omega_{4}$, and the corresponding basic mapping of the mass $M$ for this time is $P_{g}$, as shown in Figure 15(a). Once the motion in domain $\Omega_{1}$ arrives at the fourth switching point $\left(x_{4}, y_{4}\right)=(-4.5511,53.5601)$ on the elliptic boundary $\partial \Omega_{12}$ with time $t_{4}=1.0899 \mathrm{~s}$, from Figure 15(d), we have $F^{(2)}<0$ and $F^{(1)}<0$; therefore $b^{2} x_{4} y_{4}+$ $a^{2} y_{4} F^{(2)}<0$ and $b^{2} x_{4} y_{4}+a^{2} y_{4} F^{(1)}<0$ hold by simple computation, which satisfies condition (36) in Theorem 8(i). So the flow of the periodic motion will pass through the boundary $\partial \Omega_{12}$ and enter into domain $\Omega_{2}$ from domain $\Omega_{1}$, and the corresponding basic mapping of the mass $M$ for this time is $P_{c}$, as shown in Figure 15(a). The flow in domain $\Omega_{2}$ intersects the elliptic boundary $\partial \Omega_{21}$ at the fifth switching point $\left(x_{5}, y_{5}\right)=(3.7611,55.5801)$ with time $t_{5}=1.2405 \mathrm{~s}$; inequalities $F^{(1)}>0$ and $F^{(2)}>0$ follow from Figure 15(d) and $b^{2} x_{5} y_{5}+a^{2} y_{5} F^{(1)}>0$ and $b^{2} x_{5} y_{5}+a^{2} y_{5} F^{(2)}>0$ hold by simple computation, which satisfies condition (37) in Theorem 8(i). So the motion of the mass $M$ will cross $\partial \Omega_{21}$ from domain $\Omega_{2}$ into domain $\Omega_{1}$, as shown in Figure 15(a). At this time, the corresponding basic mapping of the mass $M$ is $P_{a}$. And the flow of motion of the mass $M$ in domain $\Omega_{1}$ goes back to the starting point $\left(x_{0}, y_{0}\right)$ on the velocity boundary $\partial \Omega_{14}$ at time $t_{6}=1.5225 \mathrm{~s}$, as shown in Figure 15(a). Therefore, 


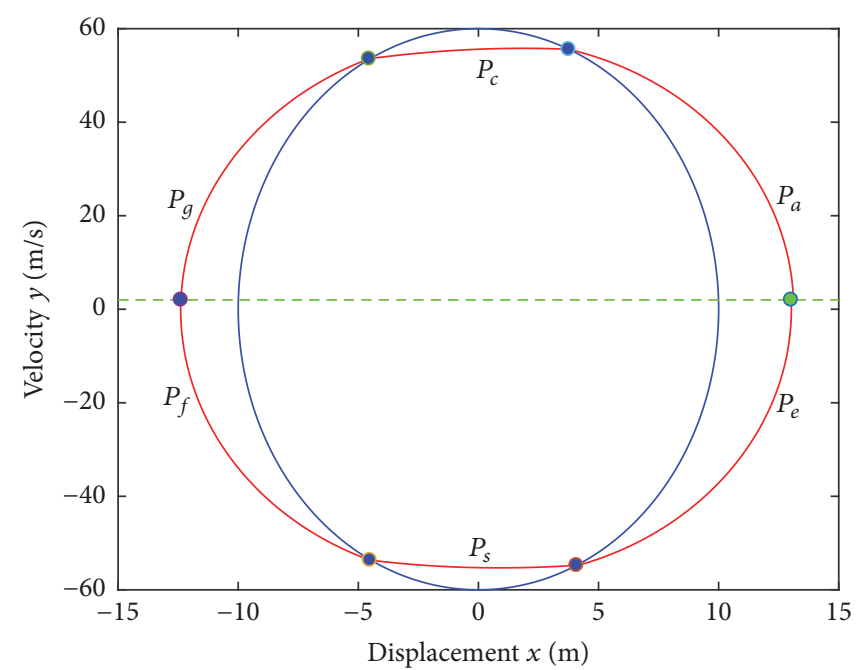

(a)

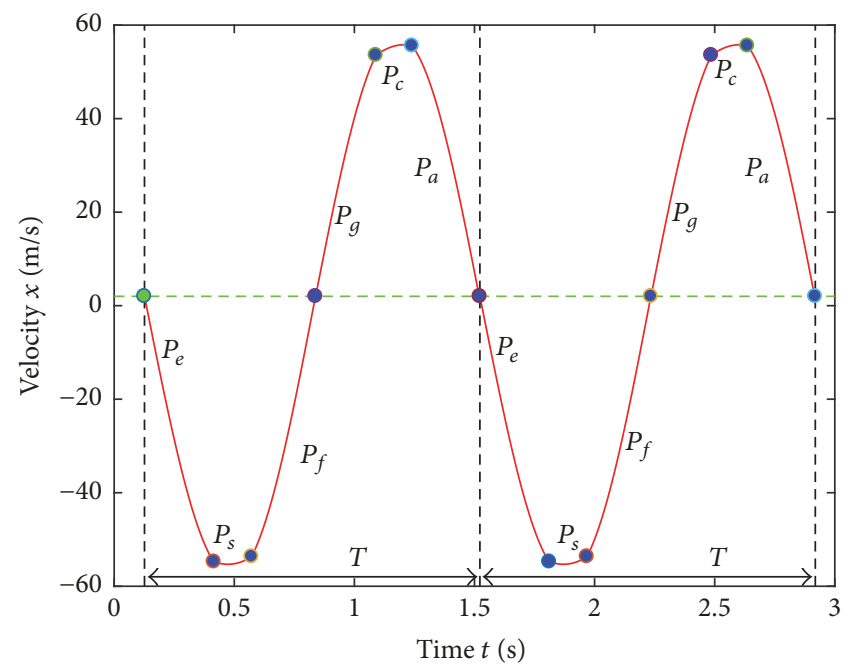

(c)

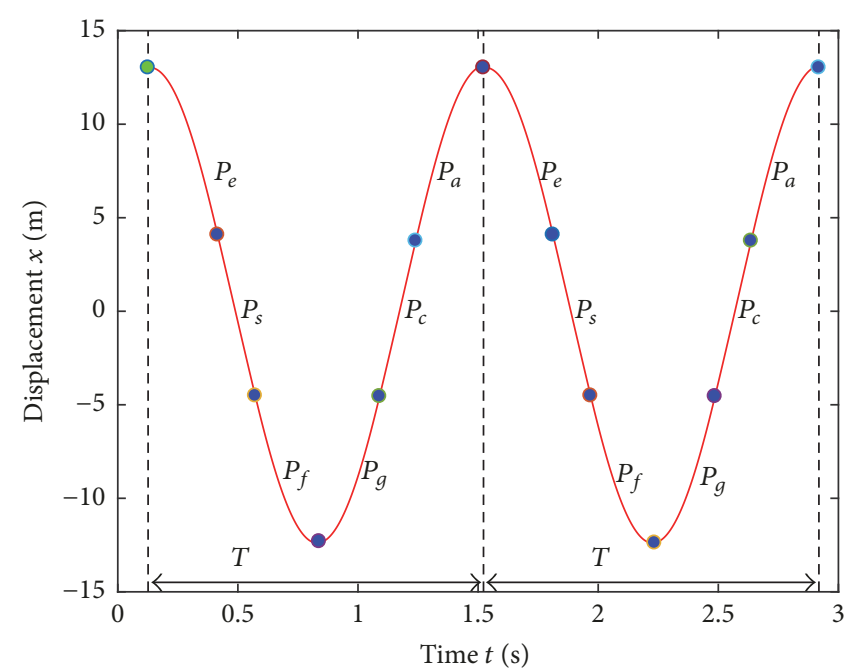

(b)

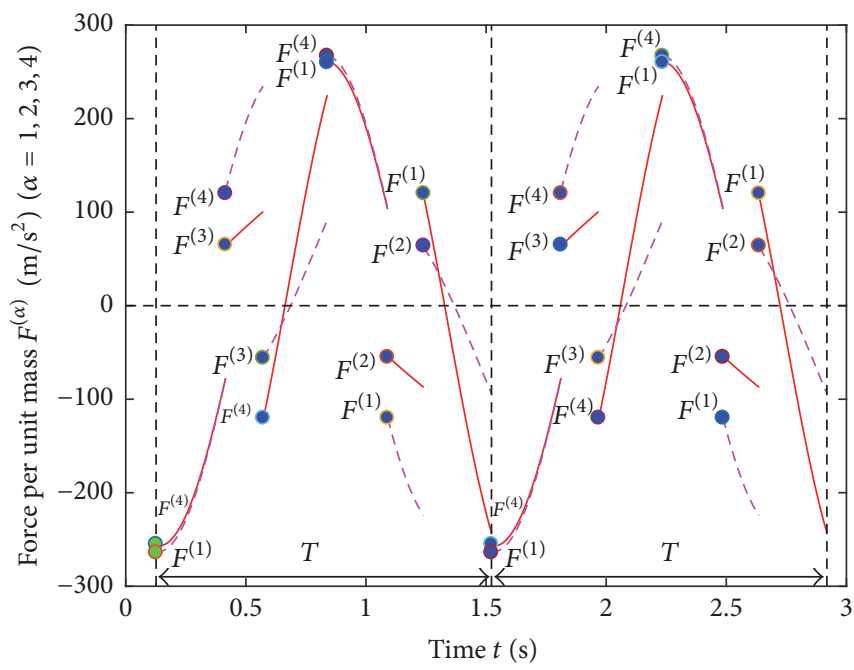

(d)

FIGURE 15: Numerical simulation of a periodic motion with a mapping structure of $P_{a c g f s e}$ : (a) phase trajectory, (b) displacement-time history, (c) velocity-time history, and (d) force $F^{(\alpha)}$-time history $\left(M=1, \mu=0.5, F_{N}=6, Q_{0}=60, \Omega=4.5, \varphi=0, B_{1}=B_{2}=10, k_{1}=24.5, k_{2}=10\right.$, $\left.\gamma_{1}=1.0, \gamma_{2}=1.0, V=2.0, a=10, b=60, t_{0}=0.1262, x_{0}=13.0205, y_{0}=2.0\right)$.

the periodic motion with a mapping structure $P_{\text {acgfse }}$ under one period is formed.

\section{Conclusions}

In this paper, the discontinuous dynamical behaviors of a friction-induced oscillator with elliptic control law and the friction force acting on the mass $M$ were investigated through the theory of flow switchability for discontinuous dynamical systems. Different domains and boundaries for such a system were defined according to the discontinuities caused by the friction and the elliptic control law. Based on the above domains and boundaries, the analytical conditions for the passable motions, sliding or stick motions, grazing motions, and the onset or vanishing of sliding or stick motions were presented through the $G$-functions of the vector fields.
The basic mappings and switching sets were introduced to describe motions in such an oscillator. Analytical conditions of periodic motions were developed by the mapping dynamics, and numerical simulations for illustration of analytical conditions and periodic motions were carried out for a better understanding of complicated dynamics of such mechanical model. There are more simulations about such an oscillator to be discussed in future.

\section{Conflicts of Interest}

The authors declare that they have no conflicts of interest.

\section{Acknowledgments}

This research was supported by the National Natural Science Foundation of China (no. 11471196, no. 11571208). 


\section{References}

[1] A. C. Luo and B. M. Rapp, "Flow switchability and periodic motions in a periodically forced, discontinuous dynamical system," Nonlinear Analysis: Real World Applications, vol. 10, no. 5, pp. 3028-3044, 2009.

[2] A. C. Luo and B. M. Rapp, "On motions and switchability in a periodically forced, discontinuous system with a parabolic boundary," Nonlinear Analysis: Real World Applications, vol. 11, no. 4, pp. 2624-2633, 2010.

[3] J. P. D. Hartog, "Forced vibrations with coulomb and viscous damping," Transactions of the American Society of Mechanical Engineers, vol. 53, pp. 107-115, 1930.

[4] E. S. Levitan, "Forced oscillation of a spring-mass system having combined Coulomb and viscous damping," The Journal of the Acoustical Society of America, vol. 32, pp. 1265-1269, 1960.

[5] A. F. Filippov, "Differential equations with discontinuous righthand side," American Mathematical Society Translations, vol. 42, no. 2, pp. 199-231, 1964.

[6] A. F. Filippov, Differential Equations with Discontinuous RightHand Sides, Kluwer Academic, Dordrecht, The Netherlands, 1988.

[7] K. Popp, N. Hinrichs, and M. Oestreich, "Dynamical behaviour of a friction oscillator with simultaneous self and external excitation," Sadhana, vol. 20, no. 2-4, pp. 627-654, 1995.

[8] M. Oestreich, N. Hinrichs, and K. Popp, "Bifurcation and stability analysis for a non-smooth friction oscillator," Archive of Applied Mechanics, vol. 66, no. 5, pp. 301-314, 1996.

[9] M. Kunze, T. Kupper, and J. You, "On the application of KAM theory to discontinuous dynamical systems," Journal of Differential Equations, vol. 139, no. 1, pp. 1-21, 1997.

[10] J. Awrejcewicz and P. Olejnik, "Stick-slip dynamics of a twodegree-of-freedom system," International Journal of Bifurcation and Chaos, vol. 13, no. 4, pp. 843-861, 2003.

[11] J. A. Cid and L. Sanchez, "Periodic solutions for second order differential equations with discontinuous restoring forces," Journal of Mathematical Analysis and Applications, vol. 288, no. 2, pp. 349-364, 2003.

[12] A. Jacquemard and M. A. Teixeira, "Periodic solutions of a class of non-autonomous second order differential equations with discontinuous right-hand side," Physica D: Nonlinear Phenomena, vol. 241, no. 22, pp. 2003-2009, 2012.

[13] T. Kupper and S. Moritz, "Generalized Hopf bifurcation for non-smooth planar systems," Philosophical Transactions Mathematical Physical, vol. 359, no. 1789, pp. 2483-2496, 2001.

[14] Y. Zou, T. Kupper, and W. J. Beyn, "Generalized Hopf bifurcation for planar Filippov systems continuous at the origin," Journal of Nonlinear Science, vol. 16, no. 2, pp. 159-177, 2006.

[15] M. Pascal, "Sticking and nonsticking orbits for a two-degreeof-freedom oscillator excited by dry friction and harmonic loading," Nonlinear Dynamics, vol. 77, no. 1-2, pp. 267-276, 2014.

[16] A. Stefaïski, J. Wojewoda, and K. Furmanik, "Experimental and numerical analysis of self-excited friction oscillator," Chaos, Solitons and Fractals, vol. 12, no. 9, pp. 1691-1704, 2001.

[17] U. Andreaus and P. Casini, "Friction oscillator excited by moving base and colliding with a rigid or deformable obstacle," International Journal of Non-Linear Mechanics, vol. 37, no. 1, pp. 117-133, 2002.

[18] J. Awrejcewicz and L. Dzyubak, "Conditions for chaos occurring in self-excited 2-DOF hysteretic system with friction," in
Proceedings of the Conference on Dynamical Systems Theory and Applications, 2005.

[19] G. Csernak and G. Stepan, "On the periodic response of a harmonically excited dry friction oscillator," Journal of Sound Vibration, vol. 295, no. 3-5, pp. 649-658, 2006.

[20] B. C. Gegg, A. C. Luo, and S. C. Suh, "Grazing bifurcations of a harmonically excited oscillator moving on a time-varying translation belt," Nonlinear Analysis: Real World Applications, vol. 9, no. 5, pp. 2156-2174, 2008.

[21] A. C. J. Luo, "Impact dynamics of a constrained massspring-damper system," International Mechanical Engineering Congress, vol. 9, pp. 1-8, 2012.

[22] Y. Guo and A. C. Luo, "Parametric analysis of bifurcation and chaos in a periodically driven horizontal impact pair," International Journal of Bifurcation and Chaos, vol. 22, no. 11, pp. 1-19, 2012.

[23] A. C. Luo and S. Thapa, "Periodic motions in a simplified brake system with a periodic excitation," Communications in Nonlinear Science and Numerical Simulation, vol. 14, no. 5, pp. 2389-2414, 2009.

[24] A. C. J. Luo and L. Chen, "Periodic motions and grazing in a harmonically forced, piecewise, linear oscillator with impacts," Chaos, Solitons \& Fractals, vol. 24, no. 2, pp. 567-578, 2005.

[25] X. Fu and Y. Zhang, "Stick motions and grazing flows in an inclined impact oscillator," Chaos, Solitons \& Fractals, vol. 76, pp. 218-230, 2015.

[26] J. Sun, W. Xu, and Z. Lin, "Research on the reliability of friction system under combined additive and multiplicative random excitations," Communications in Nonlinear Science and Numerical Simulation, vol. 54, pp. 1-12, 2018.

[27] Z. Q. Lu, J. M. Li, H. Ding, and L. Q. Chen, "Analysis and suppression of a self-excitation vibration via internal stiffness and damping nonlinearity," Advances in Mechanical Engineering, vol. 9, no. 12, 2017.

[28] D. J. Simpson, "Grazing-sliding bifurcations creating infinitely many attractors," International Journal of Bifurcation and Chaos, vol. 27, no. 12, 15 pages, 2017.

[29] C. E. da Silva, P. R. da Silva, and A. Jacquemard, "Sliding solutions of second-order differential equations with discontinuous right-hand side," Mathematical Methods in the Applied Sciences, vol. 40, no. 14, pp. 5295-5306, 2017.

[30] O. Makarenkov, "A new test for stick-slip limit cycles in dryfriction oscillators with a small nonlinearity in the friction characteristic," Meccanica, vol. 52, no. 11-12, pp. 2631-2640, 2017.

[31] Y. Ma, S. Yu, and D. Wang, "Vibration analysis of an oscillator with non-smooth dry friction constraint," Journal of Vibration and Control, vol. 23, no. 14, pp. 2328-2344, 2017.

[32] S. D. Yu and B. C. Wen, "Vibration analysis of multiple degrees of freedom mechanical system with dry friction," Proceedings of the Institution of Mechanical Engineers, Part C: Journal of Mechanical Engineering Science, vol. 227, no. 7, pp. 1505-1514, 2013.

[33] M. Bernardo, P. Kowalczyk, and A. Nordmark, "Sliding bifurcations: a novel mechanism for the sudden onset of chaos in dry friction oscillators," International Journal of Bifurcation and Chaos, vol. 13, no. 10, pp. 2935-2948, 2003.

[34] U. Galvanetto, "Sliding bifurcations in the dynamics of mechanical systems with dry friction - Remarks for engineers and applied scientists," Journal of Sound and Vibration, vol. 276, no. 1-2, pp. 121-139, 2004. 
[35] P. Kowalczyk and M. Bernardo, "Two-parameter degenerate sliding bifurcations in Filippov systems," Physica D: Nonlinear Phenomena, vol. 204, no. 3-4, pp. 204-229, 2005.

[36] P. Kowalczyk and P. T. Piiroinen, "Two-parameter sliding bifurcations of periodic solutions in a dry-friction oscillator," Physica D: Nonlinear Phenomena, vol. 237, no. 8, pp. 1053-1073, 2008.

[37] M. Guardia, S. J. Hogan, and T. M. Seara, "An analytical approach to codimension-2 sliding bifurcations in the dryfriction oscillator," SIAM Journal on Applied Dynamical Systems, vol. 9, no. 3, pp. 769-798, 2010.

[38] A. C. Luo, "A theory for non-smooth dynamic systems on the connectable domains," Communications in Nonlinear Science and Numerical Simulation, vol. 10, no. 1, pp. 1-55, 2005.

[39] A. C. Luo, "The mapping dynamics of periodic motions for a three-piecewise linear system under a periodic excitation," Journal of Sound and Vibration, vol. 283, no. 3-5, pp. 723-748, 2005.

[40] A. C. Luo, "Imaginary, sink and source flows in the vicinity of the separatrix of non-smooth dynamic systems," Journal of Sound and Vibration, vol. 285, no. 1-2, pp. 443-456, 2005.

[41] A. C. Luo, Singularity and Dynamics on Discontinuous Vector Fields, Elsevier, Amsterdam, The Netherlands, 2006.

[42] A. C. Luo, "A theory for flow switchability in discontinuous dynamical systems," Nonlinear Analysis: Hybrid Systems, vol. 2, no. 4, pp. 1030-1061, 2008.

[43] A. C. J. Luo and B. C. Gegg, "Periodic motions in a periodically forced oscillator moving on an oscillating belt with dry friction," Journal of Computational and Nonlinear Dynamics, vol. 1, no. 3, pp. 132-168, 2006.

[44] B. C. Gegg, A. C. Luo, and S. C. Suh, "Sliding motions on a periodically time-varying boundary for a friction-induced oscillator," Journal of Vibration and Control, vol. 15, no. 5, pp. 671-703, 2009.

[45] A. C. Luo, "On flow barriers and switchability in discontinuous dynamical systems," International Journal of Bifurcation and Chaos, vol. 21, no. 1, pp. 1-76, 2011.

[46] A. C. Luo and J. Huang, "Discontinuous dynamics of a nonlinear, self-excited, friction-induced, periodically forced oscillator," Nonlinear Analysis: Real World Applications, vol. 13, no. 1, pp. 241-257, 2012.

[47] Y. Zhang and X. Fu, "On periodic motions of an inclined impact pair," Communications in Nonlinear Science and Numerical Simulation, vol. 20, no. 3, pp. 1033-1042, 2015.

[48] Y. Zhang and X. Fu, "Flow switchability of motions in a horizontal impact pair with dry friction," Communications in Nonlinear Science and Numerical Simulation, vol. 44, pp. 89-107, 2017.

[49] G. Chen and J. J. Fan, "Analysis of dynamical behaviors of a double belt friction-oscillator model," WSEAS Transactions on Mathematical, vol. 15, pp. 357-373, 2016.

[50] J. Fan, S. Xue, and G. Chen, "On discontinuous dynamics of a periodically forced double-belt friction oscillator," Chaos, Solitons \& Fractals, vol. 109, pp. 280-302, 2018.

[51] J. Fan, S. Li, and G. Chen, "On dynamical behavior of a frictioninduced oscillator with 2-DOF on a speed-varying traveling belt," Mathematical Problems in Engineering, vol. 2017, Article ID 1208563, 19 pages, 2017.
[52] S. Xue and J. Fan, "Discontinuous dynamical behaviors in a vibro-impact system with multiple constraints," International Journal of Non-Linear Mechanics, vol. 98, pp. 75-101, 2018.

[53] S. Zheng and X. Fu, "Periodic motion of the van der Pol equation with impulsive effect," International Journal of Bifurcation and Chaos, vol. 25, no. 9, Article ID 1550119, pp. 1-11, 2015.

[54] J. Fan, S. Xue, and S. Li, "Analysis of dynamical behaviors of a friction-induced oscillator with switching control law," Chaos, Solitons \& Fractals, vol. 103, pp. 513-531, 2017.

[55] X. Li and S. Song, "Impulsive control for existence, uniqueness, and global stability of periodic solutions of recurrent neural networks with discrete and continuously distributed delays," IEEE Transactions on Neural Networks and Learning Systems, vol. 24, no. 6, pp. 868-877, 2013.

[56] X. Zhang, X. Lv, and X. Li, "Sampled-data-based lag synchronization of chaotic delayed neural networks with impulsive control," Nonlinear Dynamics, vol. 90, no. 3, pp. 2199-2207, 2017.

[57] X. Lv and X. Li, "Finite time stability and controller design for nonlinear impulsive sampled-data systems with applications," ISA Transactions, vol. 70, pp. 30-36, 2017.

[58] X. Li, J. Cao, and M. Perc, "Switching laws design for stability of finite and infinite delayed switched systems with stable and unstable modes," IEEE Access, vol. 6, pp. 6677-6691, 2018.

[59] X. Li and J. Wu, "Sufficient stability conditions of nonlinear differential systems under impulsive control with statedependent delay," Institute of Electrical and Electronics Engineers Transactions on Automatic Control, vol. 63, no. 1, pp. 306-311, 2018.

[60] X. Li, M. Bohner, and C.-K. Wang, "Impulsive differential equations: periodic solutions and applications," Automatica, vol. 52, pp. 173-178, 2015.

[61] H. Li and Y. Wang, "Further results on feedback stabilization control design of Boolean control networks," Automatica, vol. 83, pp. 303-308, 2017.

[62] H. Li, L. Xie, and Y. Wang, "Output regulation of Boolean control networks," Institute of Electrical and Electronics Engineers Transactions on Automatic Control, vol. 62, no. 6, pp. 29932998, 2017.

[63] H. Li, L. Xie, and Y. Wang, "On robust control invariance of Boolean control networks," Automatica, vol. 68, pp. 392-396, 2016.

[64] H. Li, Y. Wang, and L. Xie, "Output tracking control of Boolean control networks via state feedback: constant reference signal case," Automatica, vol. 59, pp. 54-59, 2015.

[65] H. Li and Y. Wang, "Lyapunov-based stability and construction of Lyapunov functions for Boolean networks," SIAM Journal on Control and Optimization, vol. 55, no. 6, pp. 3437-3457, 2017.

[66] H. Li, P. Song, and Q. Yang, "Pinning control design for robust output tracking of $k$-valued logical networks," Journal of The Franklin Institute, vol. 354, no. 7, pp. 3039-3053, 2017.

[67] Y. Zheng, H. Li, X. Ding, and Y. Liu, "Stabilization and set stabilization of delayed Boolean control networks based on trajectory stabilization," Journal of The Franklin Institute, vol. 354, no. 17, pp. 7812-7827, 2017.

[68] H. Li and Y. Wang, "Robust stability and stabilisation of Boolean networks with disturbance inputs," International Journal of Systems Science, vol. 48, no. 4, pp. 750-756, 2017.

[69] P. Senqupta, "Elliptic rendezvous in the chaser satellile frame," Journal of the Astronautical Science, vol. 59, pp. 216-236, 2012. 
[70] D. E. Chang, D. F. Chichka, and J. E. Marsden, "Lyapunovbased transfer between elliptic Keplerian orbits," Discrete and Continuous Dynamical Systems - Series B, vol. 2, no. 1, pp. 57-67, 2012.

[71] H. Yan, I. M. Ross, and K. T. Alfriend, "Pseudospectral feedback control for three-axis magnetic attitude stabilization in elliptic orbits," Journal of Guidance, Control, and Dynamics, vol. 30, no. 4, pp. 1107-1115, 2013.

[72] K. D. Do, "Formation control of multiple elliptic agents with limited sensing ranges," Asian Journal of Control, vol. 14, no. 6, pp. 1514-1526, 2012. 


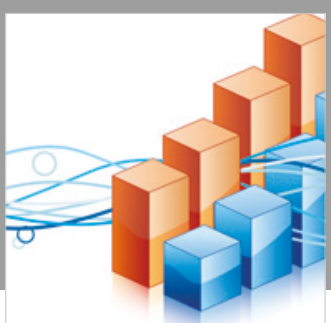

Advances in

Operations Research

\section{-n-m}
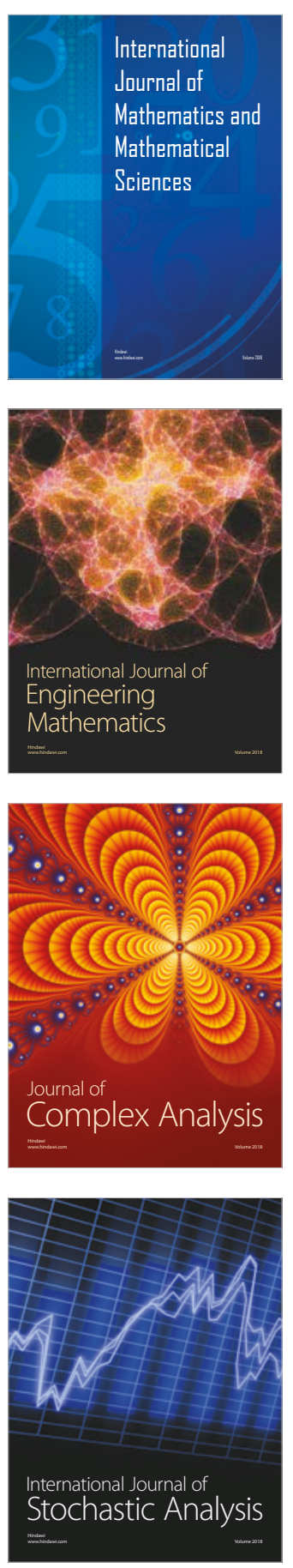
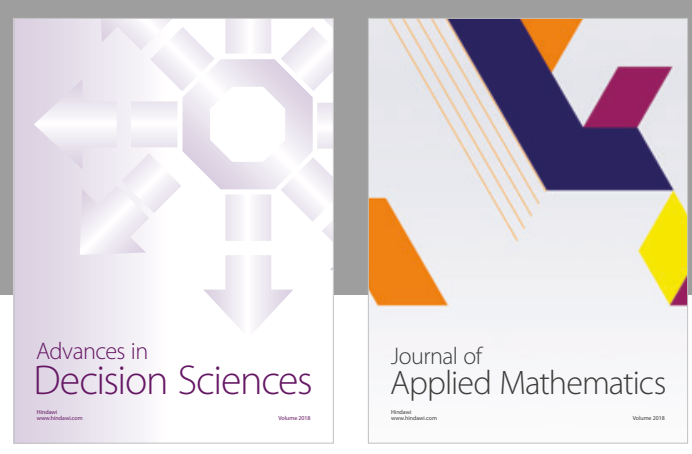

Journal of

Applied Mathematics
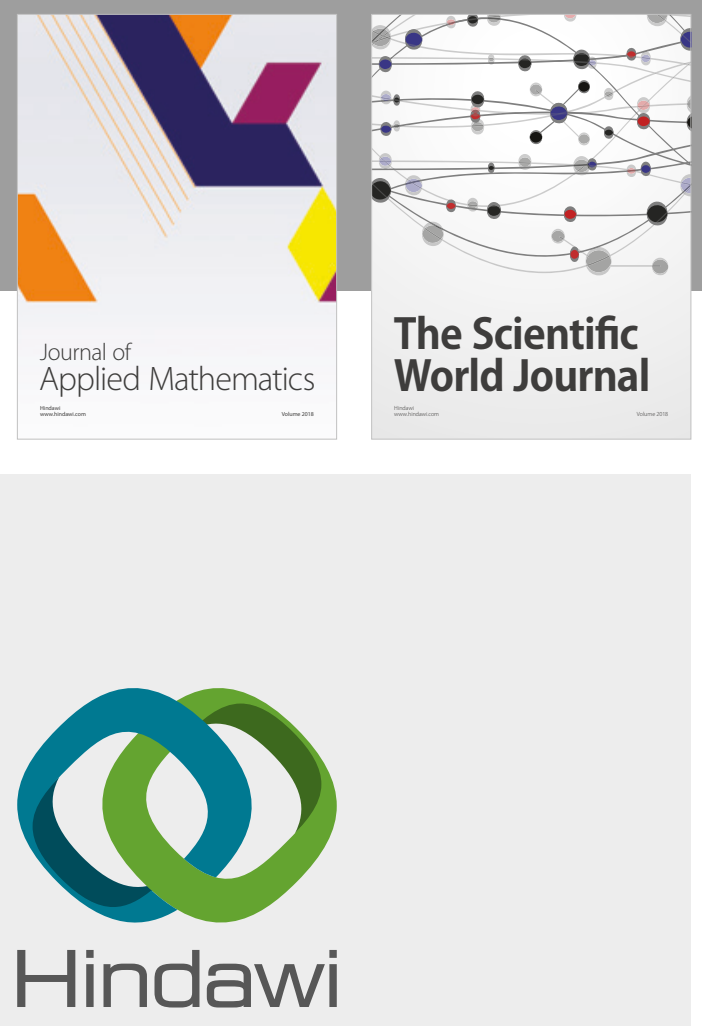

Submit your manuscripts at

www.hindawi.com

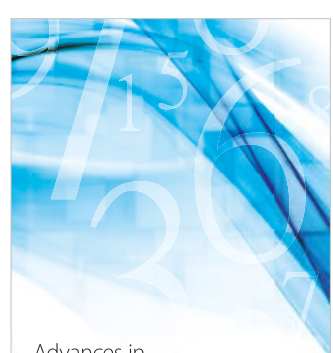

Advances in
Numerical Analysis
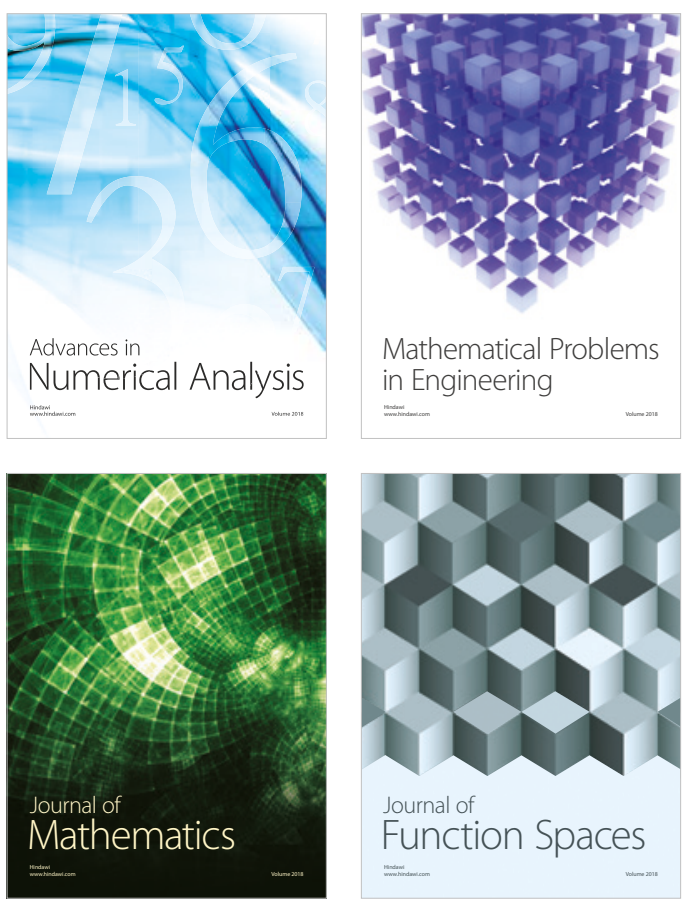

Mathematical Problems in Engineering

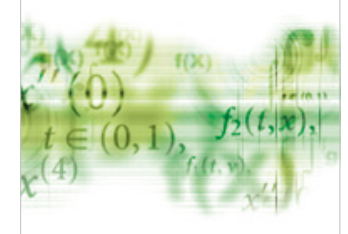

International Journal of

Differential Equations

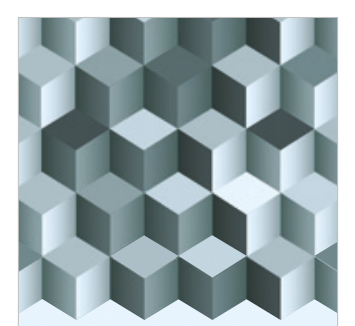

Journal of

Function Spaces

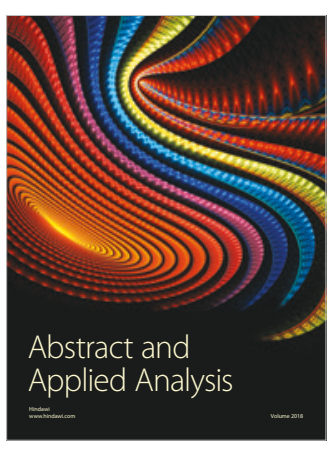

The Scientific

World Journal

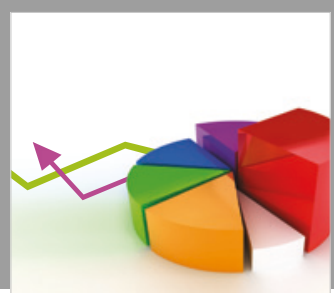

Journal of

Probability and Statistics
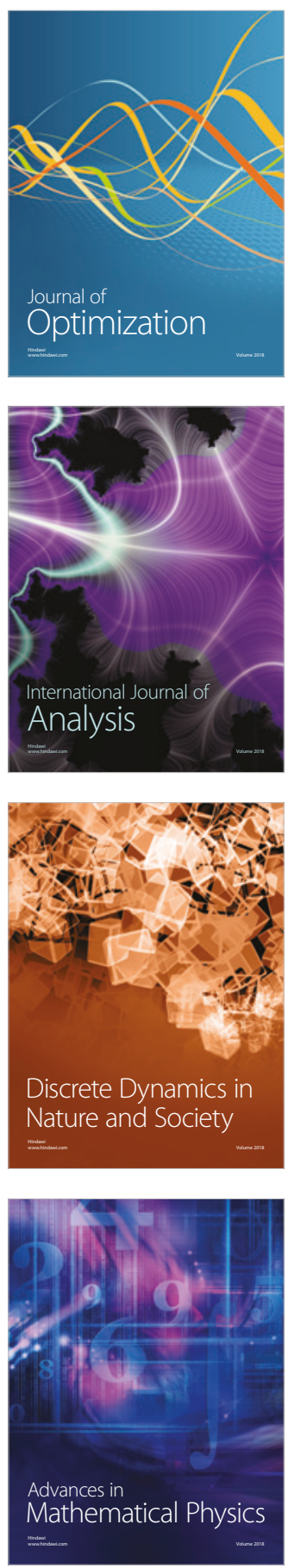